\title{
RESPONSE ANALYSIS AND MODELING OF HIGH TEMPERATURE SUPERCONDUCTOR EDGE TRANSITION BOLOMETERS
}

\author{
Mehdi FARDMANESH \\ Electrical and Electronics Engineering Dept., Bilkent University, 06800 Ankara, Turkey
}

\section{INTRODUCTION}

One of the promising devices made of high temperature superconducting (HTSC) materials are edge transition bolometers. Since the discovery of high-temperature superconductors, many works have been focused on the application of these materials in different types of bolometers for the near to far infrared wavelength regime [1]-[9]. They can be used to detect electromagnetic radiation over the whole spectrum from x-ray to the farinfrared [1], [9]-[13]. The superconductor bolometers typically consist of patterned thin or thick superconducting films deposited on crystalline substrates such as $\mathrm{MgO}, \mathrm{SrTiO}_{3}$, and $\mathrm{LaAlO}_{3}$. Their operation is based upon their steep drop in the resistance, $\mathrm{R}$, at their transition temperature, $T_{c}$. The detector is typically held at a temperature close to the middle of the superconducting transition, where the $\mathrm{dR} / \mathrm{dT}$ is maximum. When the detector is illuminated its temperature rises by an amount proportional to the input radiation power resulting in a $\Delta R$. The response obtained by the above mechanism is the so called the bolometric, or equilibrium response, as opposed to typically faster non-bolometric or intrinsic response caused by other mechanisms such as direct depairing. A typical response of an YBCO sample versus temperature at low frequencies is shown in Figure 1.1.

The responsivity versus modulation frequency and versus the temperature of edgetransition superconductive bolometers has been investigated and reported in different works [7]-[9], [14]-[18]. For example by use of a dc bias current the change in the resistance of the edge transition bolometer due to the absorbed radiation power can be measured. The detected signal obtained by this biasing can be bolometric, non-bolometric or a combination of both. Since the fabrication and testing of the first detectors, there have been many debates on the mechanism of the response in these devices. This is while the response at low frequencies of superconductor edge transition bolometer is commonly agreed to be mainly bolometric. But there have also been reports that the low frequency response of some samples is also enhanced strongly by an intrinsic response [19]-[21]. This has been reported to be dependent on bias 


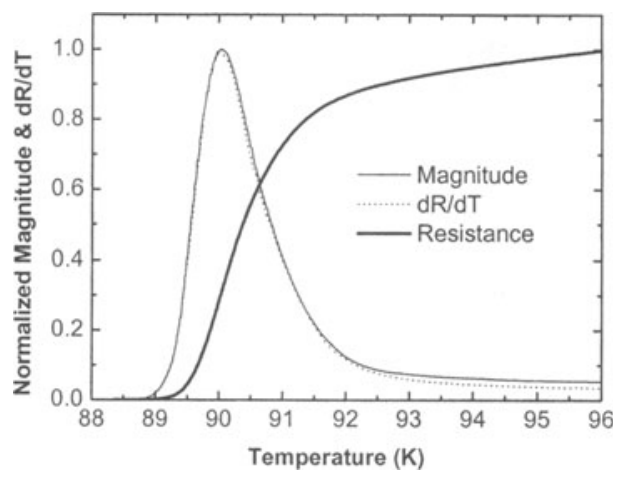

Figure 1.1. Normalized resistance and magnitude of the response at $20 \mathrm{~Hz}$ vs. temperature of $20 \mu \mathrm{m} \times 100 \mu \mathrm{m}$ area and $5 \mu \mathrm{m}$ wide meander line pattern on $1 \mathrm{~mm}$ thick $\mathrm{LaAlO}_{3}$ substrate.

current, radiation, and temperature [19]-[21]. The interpretation and the analysis of the above is strongly dependent on the thermal properties of the substrate and its dimensions, the superconducting pattern, the modulation frequency regime with respect to the effective thermal diffusion length into the substrate, and electrical and the thermal operating conditions of the detectors [7], [18].

Major effects of the substrate-holder and substrate-film thermal boundary resistance on the response of this kind of bolometers are also reported [7], [9], [14]-[18]. For superconductive edge-transition bolometers with flat crystalline substrates directly in contact with the cold-finger, the effects of limited dc thermal conductance, $G(0)$, and Joule heating on the response of the detectors have been misleading factor, resulting in unrealistic values for responsivities and detectivities [22]-[23]. This is particularly so for the low and midrange modulation frequency response of detectors with large area superconductive meander line patterns on crystalline substrates such as $\mathrm{MgO}, \mathrm{LaAlO}_{3}$, and $\mathrm{SrTiO}_{3}$ [7], [24]. The above effects are strongly dependent on the dimensions of the pattern of the superconducting film with respect to that of the substrate, and the electrical connections [25]. The observed anomalies in studying these bolometers, also can also provide insight into the heat conduction process in these devices, and can be used to study both the superconducting films and heat flow across interfaces.

Thermal modeling is essential to analyze and predict the response of the bolometers to radiation signals. To perform a thermal design of a bolometer, it is also necessary to find the thermophysical properties of the superconducting film, substrate and the interfaces at the operating temperature. Most of the contemplated uses of superconducting bolometers involve mechanical chopping of the incident light. Hence frequencies of up to a few $\mathrm{kHz}$ are of interest for many applications. The magnitude and phase of the IR-response of YBCO superconductor detectors versus modulation frequencies from $0.5 \mathrm{~Hz}$ to $100 \mathrm{kHz}$ are studied in this chapter, and found that the observed range of frequency dependence requires a model that includes the thermal boundary resistances, and proper consideration of different regimes of heat flow in the substrate. The edge transition bolometers can be classified as small area pattern (micro-bridges) and large area pattern samples with respect to the substrate dimensions and the substrate thermal parameters. The electrical connections can also be classified as voltage biased (current response) and current biased (voltage response) four (or two) probe configurations [25]. The samples considered in this chapter mostly carry large area 
superconductive patterns compared to the thickness of the substrates and the voltage-response of the bolometers is studied.

The thin film samples studied in this chapter are made of 120-550 nm thick superconducting $\mathrm{YBa}_{2} \mathrm{Cu}_{3} \mathrm{O}_{7-x}$ films on crystalline $\mathrm{SrTiO}_{3}, \mathrm{LaAlO}_{3}$, and $\mathrm{MgO}$ substrates. The films are deposited using either off-axis dc planar magnetron sputtering or PLD techniques. Details on the deposition parameters and the properties of the films are contained in References [25]-[28]. The Y-Ba-Cu-O compound with 123 and 124 stochiometries are used for fabrication of the thick film devices, post annealed in argon and oxygen ambient resulting granular superconductive films with a 123 phase [29]. The thick film samples are screen printed patterns on polycrystalline $\mathrm{MgO}$ substrates using hybrid technology. Sample substrates have $0.25 \mathrm{~mm}$ to $1 \mathrm{~mm}$ thicknesses.

The investigated IR-response measurement versus temperature, and IR-response versus modulation frequency in this chapter are measured simultaneously avoiding any artifacts due to the shift in the $T_{c}$, either caused by the thermal cycling or the Joule heating in the device. These aspects will be discussed in details in section 6 . The low noise measurements in fourprobe configuration are performed using a current source, consisting of a battery, and a bias resistor. The investigated data here is taken using lock-in amplifiers allowing the measurements of both the magnitude and phase of the response.

After studying the response characteristics in the low and midrange frequencies of YBCO edge transition bolomters in section 2, a comprehensive model and its close form solution are discussed in section 3. Section 4 discusses the anomalies in the response and the deviation of the phase of the response versus temperature, from the expected values. Section 5 describes a physical thermal model for the heat propagation mechanism based on the electron- phonon interactions correlated to the absorbed radiation energy, explaining the reported anomalies in the phase of the response versus temperature discussed in the preceding section. Section 6 discusses the dc characteristics of the superconductor edge transition bolometers and the artifacts due to the Joule heating, ambient pressure, and the microstructure, which affect the responsivity of the detectors. The noise characteristics of the bolometers are studies and classified in section 7. Section 8 applies the results from the thermal modeling and study of the noise characteristics of the bolometers discussed in the preceding section to determine and control the responsivity and detectivity limits of the edge transition bolometers through the design parameters.

\section{ANALYSIS OF LOW AND MIDRANGE MODULATION FREQUENCY RESPONSE AND THE INTERFACE EFFECTS}

In the basic model for the bolometer [4], [7], the responsivity, $r_{v}$, defined as the ratio of the voltage signal to the input power, is:

$r_{v}=\frac{\eta I}{G+j 2 \pi f C} \frac{d R}{d T}$,

where $\mathrm{I}$ is the $\mathrm{dc}$ bias current, $\mathrm{dR} / \mathrm{dT}$ is the slope of the resistance versus temperature curve at the bias (or operating) temperature, and $\eta=W / P_{t}$, with $W$ the incident power absorbed by the bolometer, and $P_{t}$ the total incident radiation power at the surface of the detector. $G$ is the total thermal conductance between the bolometer and its environment, and $\mathrm{C}$ is the heat capacity of the bolometer. A sample configuration in contact with the holder is shown in Figure 2.1.

As given in equation (2.1), $\mathrm{r}_{\mathrm{v}}$ is a complex number with a magnitude of: 


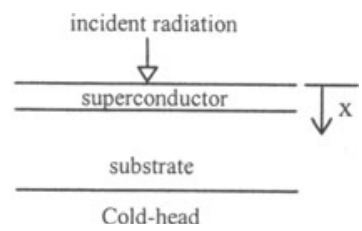

Figure 2.1. Typical experimental configuration of a superconductor bolometer on ceramic substrate, with contact to a holder (cold-head).

$$
\left|\mathrm{r}_{\mathrm{v}}\right|=\frac{\eta \mathrm{I}}{\mathrm{G} \sqrt{1+(2 \pi \mathrm{f \tau})^{2}}} \frac{\mathrm{dR}}{\mathrm{dT}}
$$

where $\tau=\mathrm{C} / \mathrm{G}$, and $\mathrm{I}$ is the constant dc bias current. Effects due to the variation of the bias current have been studied and can be found in Ref. [7]. If one assumes $G$ and $C$ are constant and independent of modulation frequency, then based on the above model, at frequencies where $2 \pi f \tau » 1$, the voltage response of a bolometer exposed to incident radiation should scale as $\mathrm{f}^{-1}$. The phase of the response in any frequency range is:

$$
\theta=\tan ^{-1}\left(\frac{-2 \pi \mathrm{fC}}{\mathrm{G}}\right)=\tan ^{-1}(-2 \pi \mathrm{f} \tau)
$$

For the studied samples in this chapter $\tau$ is typically on the order of $0.3 \mathrm{~S}$, hence the $2 \pi f \tau \gg 1$ regime holds at as low as a few $\mathrm{Hz}$, where the model above works quite well [7]. However, at frequencies in the range of $100 \mathrm{~Hz}$ and above, the measured response of almost all of the investigated samples deviates significantly from the values predicted by the above model [7], [26]. Instead of a $\mathrm{f}^{-1}$ dependence, there is a $\mathrm{f}^{-1 / 2}$ within the mid frequency range, as shown in Figure 2.2 for a sample with relatively large area pattern with respect to its substrate thickness. The thermal and geometrical parameters of this sample as well as other considered samples in this chapter are given in Table 2.1. To study this frequency behavior, an equivalent circuit model for the bolometric response to radiation signals for modulation frequencies ranging from dc to high values is considered. As shown in the circuit model in Figure 2.3, the response is determined by the total heat capacity of the film as a lumped element, and the film-substrate thermal boundary resistance as well as the thermal constants of the substrate and the interfaces.

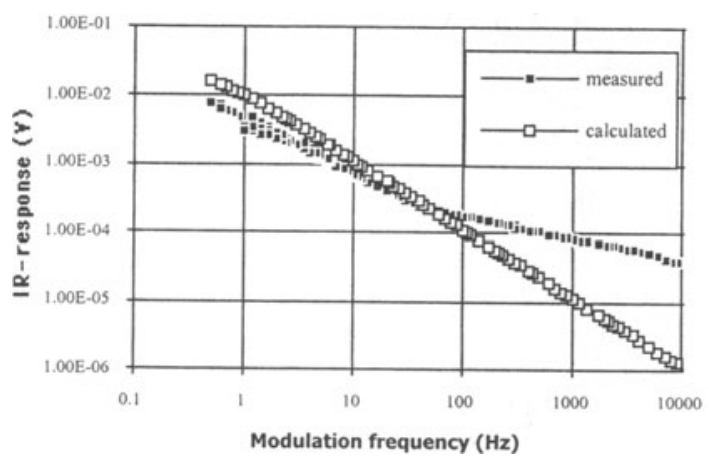

Figure 2.2. Measured and calculated IR-response versus modulation frequency of sample $064-04 \mathrm{~b}$, at $81.7 \mathrm{~K}$ with $0.68 \mathrm{~mA}$ dc bias current and $2.13 \mathrm{~mW} / \mathrm{cm}^{2}$ radiation intensity. 
Table 2.1. The dimensions of the substrates are about $0.5 \mathrm{~cm}$ by $1 \mathrm{~cm}$ for all the samples. $d_{s}$ is the thickness of the substrate, $d_{f}$ is the thickness of the YBCO film, and A is the total area enclosed by the meander line pattern and is roughly twice the area of the meander line. The values of $G$ are obtained at temperatures above $T_{c}$.

\begin{tabular}{lcccccccc}
\hline $\begin{array}{l}\text { sample } \\
\text { number }\end{array}$ & $\begin{array}{c}\text { substrate } \\
\text { material }\end{array}$ & $\begin{array}{c}\mathrm{d}_{\mathrm{f}} \\
(\mathrm{nm})\end{array}$ & $\begin{array}{c}\mathrm{d}_{\mathrm{s}} \\
(\mathrm{cm})\end{array}$ & $\begin{array}{c}\mathrm{A} \\
\left(\mathrm{cm}^{2}\right)\end{array}$ & $\begin{array}{c}\text { area of the } \\
\text { meander line }\end{array}$ & $\begin{array}{c}\mathrm{T}_{\mathrm{c} \text {-onset }} \\
(\mathrm{K})\end{array}$ & $\begin{array}{c}\mathrm{R}(300 \mathrm{~K}) \\
(\mathrm{k})\end{array}$ & $\begin{array}{c}\mathrm{G} \\
(\mathrm{W} / \mathrm{K})\end{array}$ \\
\hline $064-02 \mathrm{a}$ & $\mathrm{MgO}$ & $120-130$ & 0.025 & 0.075 & $90 \mu \mathrm{m} \times 3.35 \mathrm{~cm}$ & 87 & 13.5 & $33 \times 10^{-3}$ \\
$064-02 \mathrm{~b}$ & $\mathrm{MgO}$ & $120-130$ & 0.025 & 0.0168 & $50 \mu \mathrm{m} \times 1.9 \mathrm{~cm}$ & 86 & 11.2 & $15.5 \times 10^{-3}$ \\
$064-03 \mathrm{a}$ & $\mathrm{MgO}$ & $170-180$ & 0.05 & 0.075 & $90 \mu \mathrm{m} \times 3.35 \mathrm{~cm}$ & 82 & 7.7 & $11 \times 10^{-3}$ \\
$064-03 \mathrm{~b}$ & $\mathrm{MgO}$ & $170-180$ & 0.05 & 0.0168 & $50 \mu \mathrm{m} \times 1.9 \mathrm{~cm}$ & 83 & 6.5 & $5.1 \times 10^{-3}$ \\
$064-01 \mathrm{a}$ & $\mathrm{SrTiO}_{3}$ & $220-230$ & 0.05 & 0.0168 & $50 \mu \mathrm{m} \times 1.9 \mathrm{~cm}$ & 82 & 10.0 & $3 \times 10^{-3}$ \\
$061-02 \mathrm{a}$ & $\mathrm{LaAlO}_{3}$ & $540-560$ & 0.05 & 0.075 & $85 \mu \mathrm{m} \times 3.35 \mathrm{~cm}$ & 80 & 4.4 & $8 \times 10^{-3}$ \\
$064-04 \mathrm{~b}$ & $\mathrm{LaAlO}_{3}$ & $190-200$ & 0.05 & 0.075 & $90 \mu \mathrm{m} \times 3.35 \mathrm{~cm}$ & 84.5 & 17.5 & $7.8 \times 10^{-3}$ \\
\hline \hline
\end{tabular}

This section presents measurements and analysis of the magnitude and phase of the response of YBCO bolometers to radiation signals, with modulation frequencies up to $100 \mathrm{kHz}$. In this range of frequencies the only film property of importance is $\mathrm{dR} / \mathrm{dT}$. Behavior of the IRresponse versus frequency to radiation signals in this section is considered to be independent of the radiation wavelength. The effect of Joule heating due to the dc bias current on the response of the samples is also negligible for the test currents used in this section.

\section{A. Calculation of the response}

To compare measured results to equation (2.2), one should first determine the dc thermal conductance of the YBCO film to the surrounding environment, $G(0)$, which can be performed in several different ways [2], [7], [24]. The most convenient way is to make use of the slope of the resistance versus temperature curves of the films in the normal state near $T_{c}$ [24]. At high bias currents, the resistance of the bolometer increases due to power dissipation heating the film. This creates a temperature gradient across the substrate and the thermal interfaces. The increase in the resistance of the film due to the Joule heating is [5], [7]:

$\Delta \mathrm{R}=\mathrm{I}^{2} \mathrm{R} \frac{\mathrm{dR}}{\mathrm{GdT}}$

Using the measured values for $R, I$, and $d R / d T$, values of $G$ are obtained and given in Table 2.1 .

The heat capacity, $\mathrm{C}$, and the time constant, $\tau$, can be found by use of equation (2.3) as: $\tau=-(\tan \theta) / 2 \pi \mathrm{f}=\mathrm{C} / \mathrm{G}$,

where $\theta$ is the measured value of the phase of the response. Using equation (2.5) and the

Qt

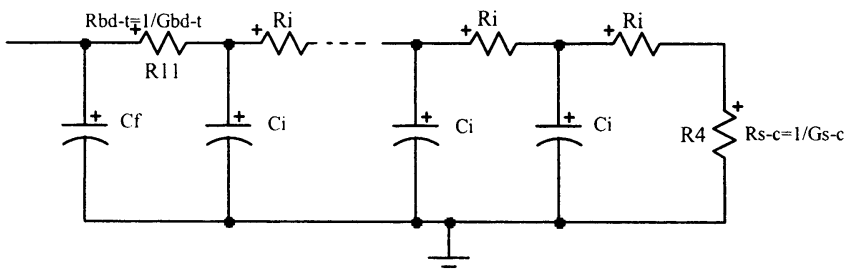

Figure 2.3. Circuit diagram of thermal model for bolometric response of a high temperature superconductor bolometer. 
measured values of $\theta$ at low frequencies, $\tau$ is calculated for sample $064-04 \mathrm{~b}$ and is found to be $\sim 0.32 \mathrm{~s}$ at frequencies less than $2 \mathrm{~Hz}$. Using the measured values of $\mathrm{G}=7.8 \mathrm{~mW} / \mathrm{K}, \tau=0.32 \mathrm{~s}$, and $\mathrm{dR} / \mathrm{dT}=3850 \Omega / \mathrm{K}$ (from $\mathrm{R}$ versus $\mathrm{T}$ curve), the response versus frequency of the sample is calculated for $\eta=1$ using equation (2.2), and is given in Figure 2.2. The calculation is done for $0.68 \mathrm{~mA}$ bias current, $2.13 \mathrm{~mW} / \mathrm{cm}^{2}$ incident radiation power with $\lambda=0.85 \mathrm{~nm}$ and an effective absorbing area of $0.03 \mathrm{~cm}^{2}$, corresponding to the values used in the measurement.

In the calculation of the response, the effect of Joule heating in the film, caused by the bias current, can also be considered. The change in the film resistance due to the input radiation power is augmented by the $I^{2} R$ heating, and is a form of positive feedback. This can enhance the response resulting in an overall responsivity of:

$r_{\mathrm{v}-\mathrm{t}}=\frac{\mathrm{r}_{\mathrm{v}}}{\left(1-\mathrm{I} \mathrm{r}_{\mathrm{v}} / \eta\right)}$,

where $r_{v}$ is given as in equation (2.1). As observed in equation (2.6), there is a stability criterion in the voltage response, which has the same form as obtained for dc biasing at zero frequency [1], [7]. Since the bias current in the samples is on the order of $10^{-3} \mathrm{~A}$, and the responsivity maximum is on the order of $10^{0} \mathrm{~V} / \mathrm{W}$, even at low frequencies, the effect of the Joule heating is negligible as mentioned before. However, in very sensitive devices or at high currents, it could become a significant factor.

The difference between the measured and calculated response at low frequencies is mostly expected to be due to the assumption of $\eta=1$. Results similar to those reported by other groups [3], [10] have been obtained for the test samples. As reported earlier and shown in Figure 2.2, the model based on constant $\mathrm{C}$ and $\mathrm{G}$, (equation (2.1)), can explain the response of the bolometers only for relatively low frequencies, where the response is limited by the thermal boundary resistance, $\mathrm{R}_{\mathrm{s}-\mathrm{c}}$, between the substrate and cold head. A more accurate analysis model requires consideration of the thermal diffusion length for the radiation-induced temperature rise into the substrate, and both thermal boundary resistances. At very low frequencies the response does follow equation (2.1) [26], where $\mathrm{C}$ and $\mathrm{G}$ are constant, and $\mathrm{G}$ is interpreted to be mainly due to the substrate-cold head thermal boundary resistance, as verified below.

\section{B. Calculations of the frequency dependent thermal parameters}

To study the thermal response of the samples, one can model the substrates as a cylinder and use the heat diffusion equation. In this model the radiation absorbing area is at one end of the cylinder [7], [18]. This assumption will turn out to be valid since the film thickness of samples is much smaller than the substrate thick nesses and the patterns of the superconducting films are relatively large in comparison to the substrate thick nesses (see Table 2.1).

Solving the diffusion equation in the substrate material, $D \nabla^{2} T=\frac{T}{t}$, as a function of the distance from the surface, $\mathrm{x}$, and time, $\mathrm{t}$, and knowing the temperature at the surface will result in [7], [18]:

$\mathrm{T}(\mathrm{x}, \mathrm{t})=\mathrm{T}_{0} \exp \left[\mathrm{i}\left(\left(\frac{\mathrm{i} 2 \pi \mathrm{f}}{\mathrm{D}}\right)^{0.5} \mathrm{x}-2 \pi \mathrm{ft}\right)\right]$,

Where $T_{0}$ is the temperature at $x=0$ and $t=0, D=K_{s} / C_{s}$ has units of centimeter square per second in the CGS system, and is called the diffusivity of the material, and $\mathrm{k}_{\mathrm{S}}$ and $\mathrm{c}_{\mathrm{S}}$ are the 
thermal conductivity and the specific heat (per unit volume) of the substrate material, respectively.

A quantity L, called the "thermal diffusion length", can be used in equation (2.7), and is defined as:

$\mathrm{L}=\left(\frac{\mathrm{D}}{\pi \mathrm{f}}\right)^{1 / 2}$.

It represents the characteristic penetration depth of the temperature variation into the substrate. Considering the thermal diffusion length as the effective length for heat flow into the substrate, the corresponding thermal conductance can be found from [2], [18]:

$\mathrm{G}=\mathrm{ak}_{\mathrm{s}} \frac{\mathrm{A}}{\mathrm{L}}$,

Where, $\mathrm{k}_{\mathrm{s}}$ is the thermal conductivity of the substrate material and "a" is a correction factor for the above approximation of the length of the heat flow. Substituting the value of $\mathrm{L}$ from equation (2.8) in equation (2.9), and using $\mathrm{D}=\frac{\mathrm{k}_{\mathrm{S}}}{\mathrm{c}_{\mathrm{s}}}$ :

$\mathrm{G}_{\mathrm{s}}=\mathrm{a} \mathrm{A} \sqrt{\mathrm{c}_{\mathrm{s}} \mathrm{k}_{\mathrm{s}} \pi \mathrm{f}}$

Thus $\mathrm{G}_{\mathrm{s}}$ will be a function of frequency and scales as $\mathrm{f}^{1 / 2}$, increasing with the frequency. Similarly, the volume of the substrate in which the temperature variation occur changes with the frequency due to the thermal diffusion length. Hence, the total heat capacity, $\mathrm{C}_{\mathrm{s}}$, can be written as:

$\mathrm{C}_{\mathrm{s}}=\mathrm{b} \mathrm{c}_{\mathrm{s}} \mathrm{A} \mathrm{L}=\mathrm{b} \mathrm{A} \sqrt{\mathrm{c}_{\mathrm{s}} \mathrm{k}_{\mathrm{s}} / \pi \mathrm{f}}$,

where $b$ is a constant to compensate for the drop of the amplitude of the temperature variation along $\mathrm{L}$. Hence, $\mathrm{C}_{\mathrm{s}}$ will also be a function of frequency and scale as $\mathrm{f}^{-1 / 2}$. Under the above assumptions, $\tau$ will be:

$\tau=\frac{\mathrm{C}_{\mathrm{s}}}{\mathrm{G}_{\mathrm{s}}}=\frac{\mathrm{b}}{\mathrm{a} \pi \mathrm{f}}$,

which will be frequency dependent, scaling as $\mathrm{f}^{-1}$.

Considering frequencies low enough to allow the total heat conductance $G_{t} \quad G_{s}$ and the total heat capacity $C_{t} \approx C_{s}$, then use of equation (2.12) in equation (2.1), results in;

$r_{v}=\frac{r^{\prime}}{1+j 2 b / a}$,

where $r^{\prime}=\frac{\eta I}{G_{t}} \frac{d R}{d T}$. Equation (2.13) shows that the phase of the response is constant and frequency independent for the frequency range where the thermal diffusion length is smaller than the thickness of the substrate. But since $G_{s}$ scales as $f^{0.5}, r^{\prime}$ will scale as $f^{0.5}$, and the magnitude of the response will be frequency dependent. This is valid at frequencies where the thermal conductance of the YBCO film, $G_{f}$, and the film-substrate interface, $G_{b d}=1 / R_{b d}$, are negligible compared to $\mathrm{G}_{\mathrm{s}}$.

The circuit diagram of the proposed thermal model of a superconducting bolometer is shown in Figure 2.3. In this model, the substrate-cold head thermal boundary resistance and the total YBCO film-substrate thermal boundary resistance are considered as $R_{\mathrm{s}-\mathrm{c}}$ and $\mathrm{R}_{\mathrm{bd}-\mathrm{t}}$, respectively. The substrate is considered to be made of small increments (thin layers of substrate material), which can be shown, by a combination of $R_{i}$ and $C_{i}$ in the circuit diagram. 
Now, for the overall frequency response analysis (from dc to high frequencies) assume that $C_{f}$ « $C_{s}$ and $R_{b d-t}$ « $R_{s} \ll R_{s-c}\left(\right.$ or, $G_{s-c}=1 / R_{s-c}$ « $G_{s}$ « $\left.G_{b d-t}=1 / R_{b d-t}\right)$, which will be shown to be the case for the samples in table 2.1 . Where $R_{b d-t}$ is the total thermal boundary resistance at the film-substrate interface. Based on these assumptions, at very low frequencies, the heat capacity of the film can be ignored compared to that of the substrate, and the total thermal conductance will be:

$\frac{1}{\mathrm{G}_{\mathrm{t}}}=\frac{1}{\mathrm{G}_{\mathrm{s}-\mathrm{c}}}+\frac{1}{\mathrm{G}_{\mathrm{s}}}+\frac{1}{\mathrm{G}_{\mathrm{bd}-\mathrm{t}}}$

Since $\mathrm{G}_{\mathrm{s}-\mathrm{c}}$ " $\mathrm{G}_{\mathrm{s}}(0)$ « $\mathrm{G}_{\mathrm{bd}-\mathrm{t}}$, we will have $\mathrm{G}_{\mathrm{t}} \approx \mathrm{G}_{\mathrm{s}-\mathrm{c}}$ which is not frequency dependent. Then, the response can be obtained from [7]:

$\Delta \mathrm{T}_{\mathrm{f}}=\frac{1}{\mathrm{G}_{\mathrm{s}-\mathrm{c}}+\mathrm{j} 2 \pi \mathrm{fC}_{\mathrm{s}}(0)} \mathrm{W}$,

Where $\Delta \mathrm{T}_{\mathrm{f}}$ is the temperature variation at the film and $\tau_{0}=\mathrm{C}_{\mathrm{s}}(0) / \mathrm{G}_{\mathrm{s}-\mathrm{c}}$. Then, ignoring the effect of the Joule heating, the responsivity of the bolometer is given by equation (2.1) with a frequency dependence of $\mathrm{f}^{-1}$, and a phase angle of -90 degree, for frequencies at which $2 \pi \mathrm{f}$ » 1 , but not so high as to limit the thermal diffusion length.

When the frequency increases to the values where the thermal diffusion length in the substrate, $\mathrm{L}$, becomes comparable to the thickness of the substrate, $\mathrm{G}$ and $\mathrm{C}$ will no longer be constant. Based on the above assumptions, $\mathrm{G} \approx \mathrm{G}_{\mathrm{s}}$ and $\mathrm{C} \approx \mathrm{C}_{\mathrm{s}}$, they are frequency dependent following equations (2.10) and (2.11) respectively, and the response is governed by:

$r_{v}=\frac{\eta I}{G_{s}+j 2 \pi f C_{s}} \frac{d R}{d T}$,

Where still $C_{f}$ « $C_{S}, G_{s}$ « $G_{b d-t}$. By applying the frequency dependence of the $G_{s}$ and $C_{s}$ in equation (2.16), the response will be in the form of equation (2.13), i.e. $r_{v \sim} f^{-1 / 2}$. As the frequency increases further, $G_{S}(f)$ increases further (or, $R_{S}(f)$ decreases) until it becomes comparable to $\mathrm{G}_{\mathrm{bd-t}}$ where $\mathrm{C}_{\mathrm{f}}$ and $\mathrm{R}_{\mathrm{bd-t}}$ can no longer be ignored. At high enough frequencies, the $\mathrm{G}_{\mathrm{s}}$ and $\mathrm{C}_{\mathrm{s}}$ can be ignored and the response will be determined by $\mathrm{G}_{\mathrm{bd}}$ (or $1 / \mathrm{R}_{\mathrm{bd}}$ ) and $\mathrm{C}_{\mathrm{f}}$ [7], [15], [17].

\section{Frequency response analysis and the experimental results}

Based on above analysis, the point at which the response start to scale as $f^{-1 / 2}$ occurs at frequencies where the thermal diffusion length, L, becomes comparable to the thickness of the substrate. Since from equation (2.8), $\mathrm{L}$ is proportional to the square root of the thermal diffusion constant, $D=k_{s} / c_{s}$, substrate materials with higher $k_{s}$, will have knee points in the

Table 2.2. Thermal characteristics of single crystal substrates

\begin{tabular}{ccccc}
\hline \hline Substrate & $k_{\mathrm{s}}(\mathrm{W} / \mathrm{K}-\mathrm{cm})$ & $\mathrm{c}_{\mathrm{s}}\left(\mathrm{J} / \mathrm{K}-\mathrm{cm}^{3}\right)$ & $\mathrm{D}\left(\mathrm{cm}^{2} / \mathrm{s}\right)$ & $\mathrm{f}_{\mathrm{L}=0.05 \mathrm{~cm}(\mathrm{~Hz})}$ \\
\hline $\mathrm{MgO}$ & $3^{\mathrm{a}}$ & $0.53^{\mathrm{a}}$ & 5.66 & 720 \\
$\mathrm{LaAlO}_{3}$ & $0.32^{\mathrm{b}}$ & 0.59 & 0.55 & 70 \\
$\mathrm{SrTiO}_{3}$ & $0.052^{\mathrm{c}}$ & $0.43^{\mathrm{c}}$ & 0.12 & 15.4 \\
\hline \hline From Ref. [2] & \multicolumn{2}{c}{ From Ref.[17] } & cFom Ref. [6]
\end{tabular}




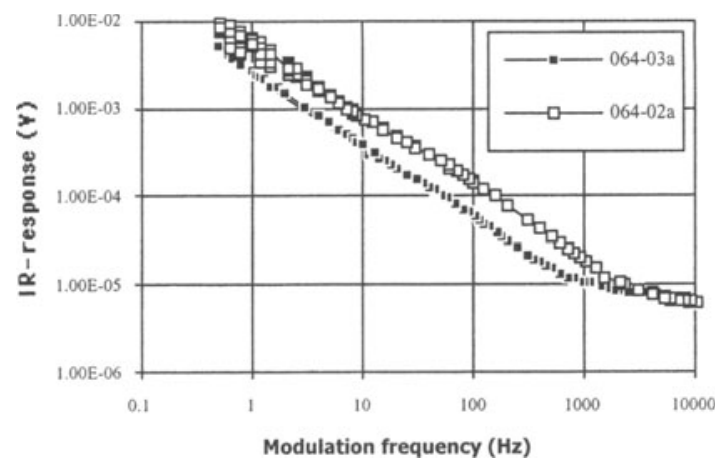

Figure 2.4. Measured IR-response versus frequency for $0.05 \mathrm{~cm}$ thick $\mathrm{MgO}$ substrate sample 064-03a and $0.025 \mathrm{~cm}$ thick $\mathrm{MgO}$ substrate sample 064-02a.

response at higher frequencies (assuming the substrate thick nesses are the same and $\mathrm{c}_{\mathrm{S}}$ of the substrates are within the same range). The values of $k_{s}, c_{s}$ and the calculated frequency of the knee point, $\mathrm{f}_{\mathrm{L}}$, of three different substrates used in this chapter are given in Table 2.2 The value of $\mathrm{c}_{\mathrm{s}}$ for $\mathrm{LaAlO}_{3}$ in Table 2.2 is derived using the phase of the low frequency response [7]-[26], and is consistent with the measured experimental values shown for $\mathrm{MgO}$ and $\mathrm{SrTiO}_{3}$. The values of $\mathrm{f}_{\mathrm{L}}$ are calculated by use of equation (2.8). For example, to find the frequency at which the thermal diffusion length is $0.05 \mathrm{~cm}$ for a $\mathrm{SrTiO}_{3}$ substrate, one can use equation (2.8) in the form of:

$\mathrm{f}_{\mathrm{L}}=\frac{\mathrm{D}}{\pi \mathrm{L}^{2}}$

For the $\mathrm{SrTiO}_{3}$ substrate from Table 2.2:

$\mathrm{D}=\frac{\mathrm{k}_{\mathrm{s}}}{\mathrm{c}_{\mathrm{s}}}=\frac{0.052}{0.43}=0.121 \mathrm{~cm}^{2} / \mathrm{s}$

Applying equation (2.18) and $\mathrm{L}=0.05 \mathrm{~cm}$ in equation (2.17), results in $\mathrm{f}_{\mathrm{L}}=15.4 \mathrm{~Hz}$ as given in Table 2.2. The measured responses versus frequency for $0.05 \mathrm{~cm}$ thick single crystal $\mathrm{LaAlO}_{3}$, $\mathrm{MgO}$, and $\mathrm{SrTiO}_{3}$ substrates are shown in Figures 2.2, 2.4, and 2.5 respectively. From the figures, the knee points in the response versus frequency are about $15 \mathrm{~Hz}, 60 \mathrm{~Hz}$, and $600 \mathrm{~Hz}$, for $0.05 \mathrm{~cm}$ thick $\mathrm{SrTiO}_{3}, \mathrm{LaAlO}_{3}$, and $\mathrm{MgO}$ substrates, respectively, in good agreement with the calculated values in the table.

To verify the above analysis and to investigate the effect of the thickness of the substrate on the frequency response, we consider two samples with identical meander line patterns on $0.25 \mathrm{~mm}$ and $0.5 \mathrm{~mm}$ thick $\mathrm{MgO}$ substrates. The response of the samples 064-02a and 06403a, are shown in Figure 2.4. As shown in the figure, the knee point is found to be dependent on the substrate thickness, and it is found not to be dependent on the pattern area. The knee point for the thinner substrate sample 64-02a occurs at higher frequencies, about $2.5 \mathrm{kHz}$ to 3 $\mathrm{kHz}$, whereas for the thicker substrate sample 64-03a, it occurs at about $700 \mathrm{~Hz}$ to $800 \mathrm{~Hz}$. Setting the thickness of the substrate equal to $\mathrm{L}$ in equation (2.17), and using the values of $\mathrm{c}_{\mathrm{S}}$ and $\mathrm{k}_{\mathrm{s}}$ from Table 2.2 for $\mathrm{MgO}$, knee points at frequencies of $720 \mathrm{~Hz}$ and $2.8 \mathrm{kHz}$ are calculated for samples $64-03 \mathrm{a}(0.05 \mathrm{~cm}$ thick) and $64-02 \mathrm{a}(0.025 \mathrm{~cm}$ thick $)$, respectively. The above calculated values of frequency agree with the break frequencies seen in Figure 2.4. 


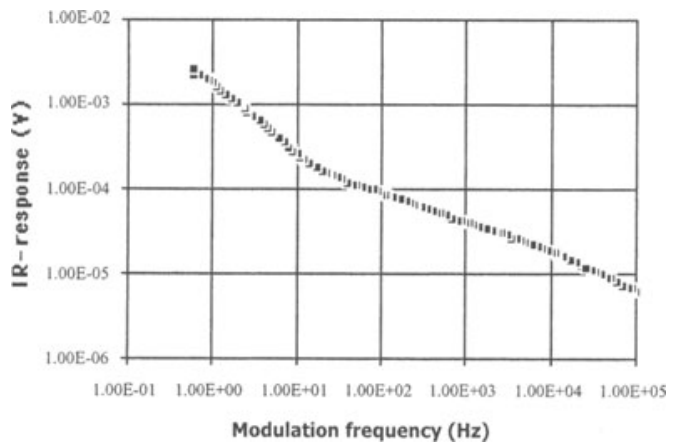

Figure 2.5. IR-response versus modulation frequency of $\mathrm{SrTiO}_{3}$ substrate sample 064-01a measured at $0.25 \mathrm{~mA}$ bias currents.

From equation (2.13), for frequencies higher than $f_{L}$, the phase should be frequency independent. Figure 2.6 (next page) shows the measured phase of the response for sample 064-01a, which confirms the position of the break frequency discussed above. The flat region in Figure 2.6 extends only to about $10 \mathrm{kHz}$, but it should continue up to the frequencies where the response and its phase starts to be governed by the effect of $\mathrm{R}_{\mathrm{bd}}$, according to our model. Since the YBCO film is just a few $100 \mathrm{~nm}$ thick, the heat capacity of the film, $\mathrm{C}_{\mathrm{f}}$, is very low, and the phase of the response should approach $0^{\circ}$ for the frequency range where the response is limited by the effect of $R_{b d}$, and continue until much higher frequencies. The frequency at which $\mathrm{R}_{\mathrm{bd}}$, the film-substrate thermal boundary resistance, becomes important varies with the thermal parameters of the substrate material. It is lower for substrate materials with higher $k_{S}$ (assuming $\mathrm{c}_{\mathrm{s}}$ of the substrates are within the same range) [7]. Using $\mathrm{c}_{\mathrm{s}}$ and $\mathrm{k}_{\mathrm{s}}$ from Table 2.2, and a typical value for $\mathrm{R}_{\mathrm{bd}}$ as $1.1 \times 10^{-3} \mathrm{~K}-\mathrm{cm}^{2} / \mathrm{W}$ [17], one can compare this with the frequency dependent thermal resistance of the substrate, as calculated from equation (2.10). The thermal resistance due to $\mathrm{R}_{\mathrm{bd}}$ is equal to the thermal resistance of $\mathrm{MgO}, \mathrm{LaAlO}_{3}$, and $\mathrm{SrTiO}_{3}$ substrates at modulation frequencies of $165.4 \mathrm{kHz}, 1.413 \mathrm{MHz}$, and $11.67 \mathrm{MHz}$, respectively. These frequencies are calculated for the case that the corresponding thermal diffusion lengths are small compared to the width of the meander lines of the superconducting pattern, which is the case in the samples of Table 2.1. For smaller patterns, the spacing between the lines should also be considered. This will result in frequencies lower than above [7].

One can estimate the frequencies at which $\mathrm{R}_{\mathrm{bd}}$ and $\mathrm{c}_{\mathrm{s}}$ would change the phase, and this is 10 to $50 \mathrm{MHz}$ range. Hence this cannot account for the drop in the phase seen in Figure 2.5. The drop in the phase at frequencies of about $10 \mathrm{kHz}$ is also observed for samples with $\mathrm{MgO}$ and $\mathrm{LaAlO}_{3}$ substrates. This departure from our model for the thermal response of the samples may be due to other effects, such as the system circuitry [7], or photo-induced conductivity changes in the YBCO film [34]. However, this analysis accurately accounts for the observed behavior below $10 \mathrm{kHz}$. To summarizes this section, the slope of the response changes at "knee" frequencies of about $15 \mathrm{~Hz}, 60 \mathrm{~Hz}$, and $600 \mathrm{~Hz}$ for $0.05 \mathrm{~cm}$ thick $\mathrm{SrTiO}_{3}, \mathrm{LaAlO}_{3}$, and $\mathrm{MgO}$ substrates, respectively, from a $\mathrm{f}^{-1}$ to a $\mathrm{f}^{0.5}$ dependence. From equation (2.1), one sees that a high $G$ value, $G » 2 \pi f c$, lowers the responsivity, and pins the phase of the response at 


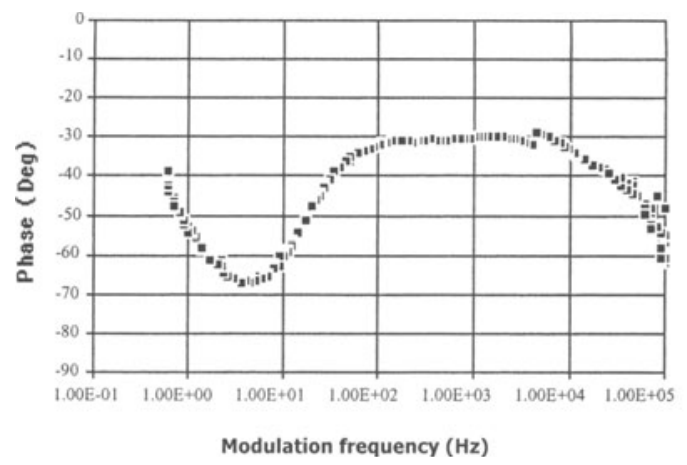

Figure 2.6. Phase of IR-response versus modulation frequency of sample 064-01a $\left(0.05 \mathrm{~cm}\right.$ thick $\mathrm{SrTiO}_{3}$ substrate).

$\theta=0^{\circ}$. A low $\mathrm{G}$ value, $\mathrm{G} \ll 2 \pi \mathrm{fc}$, gives $\theta=-90^{\circ}$, a $\mathrm{f}^{-1}$ dependence to the responsivity, and a magnitude inversely proportional to the heat capacitance of the bolometer. For this latter case a very thin substrate to reduce $\mathrm{C}$ is indicated. While $\mathrm{G}$ can be made very small by isolating the substrate (underneath the pattern) from the cold head, the minimum $G(0)$ is needed to keep the sample in the superconducting state, and within a $\Delta \mathrm{T}$ of its bias temperature. For the considered samples here with G's on the order of $10^{-2} \mathrm{~W} / \mathrm{K}$, Joule heating power on the order of $I^{2} R=10^{-2} \mathrm{~W}$ yields a shift temperature on the order of $1 \mathrm{~K}$. Hence as a practical matter, a $\mathrm{G}$ much smaller than the values in table 2.1 would present a problem in maintaining the bias temperature for currents on the order of $1 \mathrm{~mA}$.

\section{ANALYTIC THERMAL MODELING FOR DC TO MIDRANGE MODULATION FREQUENCY RESPONSE}

There has been different efforts on the response modeling of the edge-transition superconducting bolometers with and without the consideration of the effects of the thermal boundary resistance at the substrate interfaces and the self or Joule heating in the superconducting film [16], [35], [30]-[31]. Based on the above proposed effects of the introduced parameters, section 2 discussed successful analysis and prediction of the behavior of the response versus modulation frequency curves predicting the frequencies at which the slope of the curve changes (the knee frequency) [7], [16]. Though, one cannot predict and match the response in all the measured modulation frequency regimes using an analytic model. Using the analyses in section 2,one can predict and match the measured response of the samples at very low frequencies showing different considerable discrepancies at midrange and/or higher modulation frequencies [7], [16]. In many of the proposed models and the solutions, only the magnitude of the response have been considered and compared to the experimental values. This is while the phase of the response is a more sensitive characteristic of the response with respect to the model and the chosen or obtained thermal parameters of the devices [24]. In this section the results obtained from a recent closed form solution of a comprehensive one-dimensional thermal model is discussed. The results from the model are compared to the both measured values of the magnitude and phase of the response of the samples. 


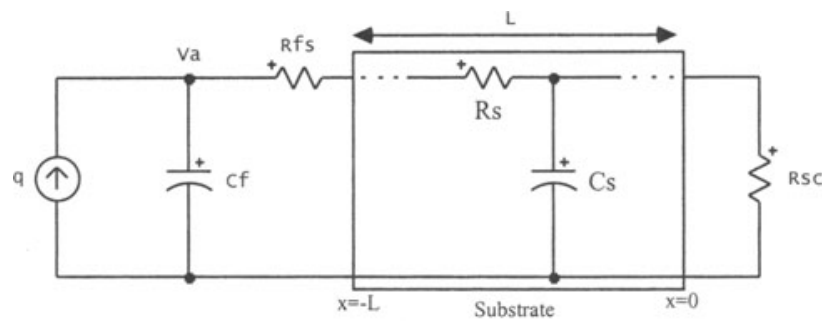

Figure 3.1. Equivalent thermophysical diagram of the bolometers in contact with the holder, Where $q$ is input radiation power, $C_{f}$ is lumped heat capacitance of the film, $R_{f s}$ and

$R_{s c}$ are the thermal boundary resistance values at the substrate interfaces.

\section{A. The Thermal Modeling}

From the given dimensions in Table 2.1, the area of the superconducting pattern of the samples is large with respect to the substrate thickness. Hence a one-dimensional model is considered and applied for the studied samples while considering all the parameters for per unit area. Because of the partial lateral heat diffusion of the coming heat wave from the film into the substrate (the spreading resistance effect), a correction factor, $\alpha$, is considered for applying to the measured $R_{\mathrm{sc}}$ values and is obtained and given for some of the samples in Table 3.1. The value of $\alpha$ increases with the increase of the thermal conductivity of the substrate material and being the highest for $\mathrm{MgO}$. It increases with decrease of the superconducting pattern area with respect to the substrate thickness as given in the table to be higher for the small pattern film on $\mathrm{SrTiO}_{3}$ compared to that of the $\mathrm{LaAlO}_{3}$ substrate sample. As given in the tables, the thickness of the superconducting films of the samples is also very small compared to that of the substrate. This allows the consideration of a lumped film assumption on top surface of the substrate in the model.

Considering the superconducting pattern as a lumped film and substrate as a continues uniform media, the thermal equivalent circuit of the samples in contact with the holder can be represented as in Figure 3.1, where $\mathrm{q}$ is the absorbed radiation intensity at the surface of the sample. In this equivalent diagram, two thermal boundary resistances are considered at the substrate interfaces. The heat capacitance of the superconducting film is also considered to be a value representing the total heat capacity of the film per unit area. The thermal conductance of the superconducting film is calculated to be negligible compared to that of the substrate and the interfaces. For samples with thicker superconducting films as for the screen-printed materials [4], the limited heat conductivity of the superconducting material same as for the substrate, should also be considered. Based on the above considerations in the model as

Table 3.1. Dimensions and the obtained thermal parameters of the samples used in the model.

\begin{tabular}{ccccccccccc}
\hline \hline $\begin{array}{l}\text { sample } \\
\text { number }\end{array}$ & $\begin{array}{c}\text { substrate } \\
\text { material }\end{array}$ & $\begin{array}{c}\mathrm{D}_{\mathrm{S}} \\
(\mathrm{cm})\end{array}$ & $\begin{array}{c}\mathrm{C}_{\mathrm{S}} \\
\left(\frac{\mathrm{J}}{\mathrm{k}-\mathrm{cm}^{3}}\right)\end{array}$ & $\begin{array}{c}\mathrm{K}_{\mathrm{s}} \\
\left(\frac{\mathrm{W}}{\mathrm{k}-\mathrm{cm}}\right)\end{array}$ & $\begin{array}{c}\mathrm{R}_{\mathrm{sc}} \\
\left(\frac{\mathrm{K}}{\mathrm{W}-\mathrm{cm}^{2}}\right)\end{array}$ & $\begin{array}{c}\alpha \\
(\mathrm{nm})\end{array}$ & $\begin{array}{c}\mathrm{A} \\
\left(\mathrm{cm}^{2}\right)\end{array}$ & $\begin{array}{c}\mathrm{R}_{\mathrm{fs}} \\
\left(\frac{\mathrm{mK}}{\mathrm{W}-\mathrm{cm}^{2}}\right)\end{array}$ & $\begin{array}{c}\mathrm{C}_{\mathrm{f}} \\
\left(\frac{\mathrm{kJ}}{\mathrm{k}-\mathrm{cm}^{2}}\right)\end{array}$ \\
\hline $064-03 \mathrm{a}$ & $\mathrm{MgO}$ & 0.05 & 0.53 & 3 & 6.82 & 2 & 170 & 0.075 & 4.4 & 642 \\
$064-01 \mathrm{a}$ & $\mathrm{SrTiO}_{3}$ & 0.05 & 0.43 & 0.052 & 5.6 & 1.7 & 220 & 0.017 & 44 & 655 \\
$061-02 \mathrm{a}$ & $\mathrm{LaAlO}_{3}$ & 0.05 & 0.59 & 0.16 & 8.82 & 1 & 550 & 0.075 & 27.5 & 131
\end{tabular}


shown in Figure 3.1, the derivation of the temperature variation and the corresponding thermal parameters for the samples under a constant bias current is presented in the following.

a) Temperature Variation in the Substrate

To approach the complete closed form solution to the model given in Figure 3.1, first one can consider the absorbed radiation power, $\mathrm{q}$, is applied directly to the surface of the substrate. This is ignoring the effects of the surface boundary conditions of $C_{f}$ and $R_{f s}$, which are representing the effect of the thermal parameters of the superconducting film at this stage. This particular assumption and the solution to it would be the case as for very low modulation frequencies. At low modulation frequencies, $\mathrm{C}_{\mathrm{f}}$ is much smaller than the contributing heat capacity of the substrate, which can be determined from equation (2.17) [7]. Also the filmsubstrate thermal boundary resistance, $\mathrm{R}_{\mathrm{fs}}$, is much smaller than that of the substrate-holder and/or the low frequency thermal resistance of the substrate, $R_{s}=1 / G_{s}$, determined from equation (2.10).

Based on the above assumptions and the steady state solution to general one dimension heat propagation equation, the temperature variation at the surface of the substrate, $\Delta \mathrm{T}$, can be obtained. Without the consideration of the effect of the superconducting film (i.e. $\mathrm{C}_{\mathrm{f}}$ and $\mathrm{R}_{\mathrm{fs}}$ ) $\Delta \mathrm{T}$ follows;

$$
\Delta \mathrm{T}=\mathrm{q} \frac{\exp (\gamma \mathrm{L})+\Gamma \exp (-\gamma \mathrm{L})}{\exp (\gamma \mathrm{L})-\Gamma \exp (-\gamma \mathrm{L})} \sqrt{\frac{1}{j \omega \mathrm{c}_{\mathrm{s}} \mathrm{k}_{\mathrm{s}}}}
$$

where $\gamma$ is the characteristic thermal impedance of the substrate material defined as;

$\gamma=\frac{1+j}{\sqrt{2}} \sqrt{\frac{\omega c_{s}}{k_{s}}}$

And;

$$
\Gamma=\frac{R_{s c}-\sqrt{\frac{1}{j \omega c_{s} k_{s}}}}{R_{s c}+\sqrt{\frac{1}{j \omega c_{s} k_{s}}}},
$$

Where $\omega$ is the angular modulation frequency, and $k_{s}$ and $c_{s}$ are the heat conductivity and the heat capacity of the substrate materials, respectively. The value of $\mathrm{R}_{\mathrm{sc}}$ can be obtained;

$\mathrm{R}_{\mathrm{sc}}=\frac{1}{\mathrm{G}(\mathrm{W} / \mathrm{K}) / \mathrm{A}}$,

Where $\mathrm{G}$ is in units of $\mathrm{W} / \mathrm{K}$ and is the total thermal conductance of the device at very low frequencies, $G(0)$, and $A$ is the film pattern area. This is based on the assumption of $G_{f s}$ " $G_{s}$ " $G_{s c}$ where $G_{f s}=1 / R_{f s}, G_{s}$ is the total heat conductance of the substrate, and $G_{s c}=1 / R_{s c}$. This assumption is verified for the samples with dimensions close to that of table 2.1 and 3.1 , which is also discussed in section 2 [7], [16], [22]-[24], [37].

\section{b) Temperature Variation at the Film}

To find the voltage response to the modulated radiation intensities, the temperature variation in the superconductive film of the samples can be obtained for all considered frequency ranges. This temperature variation corresponds to the temperature change across $\mathrm{C}_{\mathrm{f}}$ in the equivalent circuit of Figure 3.1. Hence the only temperature gradient from top of the superconducting film into the substrate would be that across the film-substrate interface, $R_{f s}$. 
Solving the one-dimensional thermal differential equation with the complete boundary conditions as shown in Figure 3.1 (i.e. with the consideration of $C_{f}$ and $R_{f s}$ ), the temperature variation caused by the radiation absorption in the film follows;

$\Delta \mathrm{T}=\mathrm{q}\left(\frac{\frac{\exp (\gamma \mathrm{L})+\Gamma \exp (-\gamma \mathrm{L})}{\exp (\gamma \mathrm{L})-\Gamma \exp (-\gamma \mathrm{L})} \sqrt{\frac{1}{j \omega \mathrm{c}_{\mathrm{s}} \mathrm{k}_{\mathrm{s}}}}+\mathrm{R}_{\mathrm{fs}}}{\frac{\exp (\gamma \mathrm{L})+\Gamma \exp (-\gamma \mathrm{L})}{\exp (\gamma \mathrm{L})-\Gamma \exp (-\gamma \mathrm{L})} \sqrt{\frac{j \omega}{\mathrm{c}_{\mathrm{s}} \mathrm{k}_{\mathrm{s}}}} \mathrm{C}_{\mathrm{f}}+1+j \omega \mathrm{C}_{\mathrm{f}} \mathrm{R}_{\mathrm{fs}}}\right)$.

Where, $\mathrm{q}$ is the absorbed radiation power in the film per unit area, and $\mathrm{C}_{\mathrm{f}}$ and $\mathrm{R}_{\mathrm{fs}}$ are the total heat capacity of the superconducting film and the thermal boundary resistance at the filmsubstrate interface per unit area, respectively.

The solution of equation (3.5) is up to frequencies where the thermal diffusion length into the superconducting film (for YBCO material) becomes smaller and/or comparable to the thickness of the film. This is while the penetration depth and/or the absorption coefficient length in the superconducting film is still considered to be much smaller than the film thickness. This frequency regime for the samples of II_Table 3.1 with a maximum film thickness of about $550 \mathrm{~nm}$ much higher than that considered here. As given in equation (3.5), at very low frequencies the denominator will approach one and the $\mathrm{R}_{\mathrm{fs}}$ will become negligible compared to the first term of the numerator leading to the results of the one boundary condition problem as given in equation (3.1). This is because at very low modulation frequencies the temperature variation (heat wave) reaches the bottom of the substrate and hence the heat capacity of the film with respect to that of the whole substrate material under the pattern, and also the thermal boundary resistance at the film-substrate with respect to $R_{S}$ and $\mathrm{R}_{\mathrm{sc}}$ become negligible [7], [37].

c) The Voltage Response and Bias Current Dependence

Considering a uniform temperature variation in the film, caused by the absorbed radiation, $\Delta \mathrm{T}$, the voltage response in a dc current bias configuration can be obtained from;

$\Delta \mathrm{V}=\mathrm{I}_{\mathrm{b}} \Delta \mathrm{T} \frac{\mathrm{dR}}{\mathrm{dT}}$,

Where $I_{b}$ is the dc bias current, $d R / d T$ is the slope of the $R$ versus $T$ curve at the bias temperature as given in II_Table 3.1 , and $\Delta \mathrm{T}$ is the temperature variation in the superconducting film as given in equation (3.5). Equation (3.6) is valid within the temperature variation range where $\mathrm{dR} / \mathrm{dT}$ can be considered to be constant. The maximum temperature variations of the films caused by the radiation is typically below about a few $\mathrm{mK}$. The transition widths of the samples are typically of a few degrees of Kelvin and hence the variation of $\mathrm{dR} / \mathrm{dT}$ and/or the non-linearity of equation (3.6) are negligible in the measured and calculated response. Though this would not be the case for devices exposed to high radiation intensities, which are normally voltage biased for further stability's [12]-[13]. The effect of the bias configuration on the response and the feedback effect of the Joule heating for each case of the voltage-biased and current-biased configurations are discussed elsewhere [25].

There is also a consecutive temperature variation due to ac Joule heating in the film caused by the resistance variation in the superconducting film due to the input radiation power. This effect on the response is in the form of a positive feedback in the used currentbiased configuration. This ac Joule heating in a first order approximation can be considered either as a dependent radiation (or heating) source in parallel to the external radiation source, 
q, shown Figure 3.1 [40], or simply as an additional term in the overall responsivity of the sample discussed in section 2. The effect of ac Joule heating in the response of the samples studied in this work is negligible for the used biased currents.

\section{B. Measured Frequency Response and the Results From the Model}

The results from the model were compared to the experimental values for the samples given in II_Table 1. The frequency response behavior of the samples was found to be strongly dependent on the film and substrate dimensions as well as on the thermal parameters, while the observed dependencies are in good agreement with the thermal model. To calculate the response using equation (3.5), the dc thermal parameters of $R_{f s}, R_{s c}, C_{f}, C_{s}$, and $K_{s}$ of the samples were determined. The measured values of the above parameters are given in II_Table 1. The values of $R_{s c}$, which are much higher than $R_{s}$ and $R_{f s}$, are measured using the method of the resistive or the dc Joule heating in the film close to the transition temperature, $T_{c}[5]$, [24], [39]. In this method a high bias current is passed through the film and the temperature rise due to the Joule heating is measured by measuring the resistance of the film. Then knowing the temperature rise and the Joule heating power the values of $\mathrm{R}_{\mathrm{sc}}$ are obtained. Also the parameters such as the film-substrate thermal boundary resistance and the thermal parameters of the substrate or the heat capacity of the samples could be obtained and verified using the fit of the results from the model to the measured values. The above values in II_Table 1 are given per unit area as used in the one-dimensional solution of the model.

The results from the model were investigated for thin film samples on crystalline $\mathrm{SrTiO}_{3}$, $\mathrm{LaAlO}_{3}$, and $\mathrm{MgO}$ substrate materials. The measured and calculated magnitude and phase of the response of the meander line patterned YBCO films on the 0.05 thick $\mathrm{SrTiO}_{3}, \mathrm{LaAlO}_{3}$, and $\mathrm{MgO}$ substrate samples are shown in figures 3.2, 3.3, and 3.4 respectively. Calculated response using the basic $\mathrm{R}-\mathrm{C}$ model in section 2 (equation (2.1)) is also plotted for samples 064-01a and 061-02Aa.

As discussed in section 2, the basic R-C model is as useful as the complete model in the very low frequency ranges where the thermal diffusion length into the substrate is much larger than the substrate thickness. This basic $\mathrm{R}-\mathrm{C}$ model is appropriate for micromachined and/or freestanding film bolometers. The $\mathrm{G}$ and $\mathrm{C}$ values of such bolometers would be constant up to
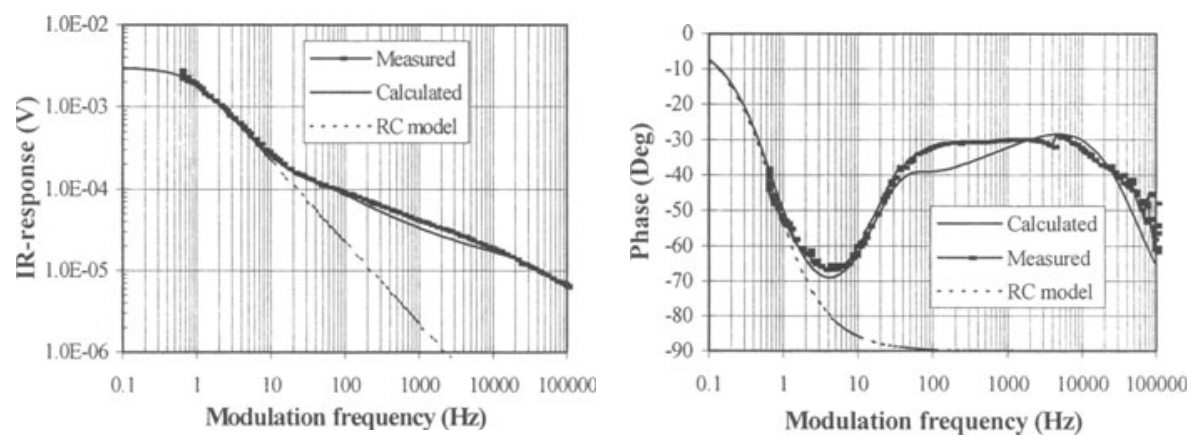

Figure 3.2. Measured and calculated magnitude (left) and phase (right) of response versus modulation frequency of the $\mathrm{SrTiO}_{3}$ substrate sample 064-01a at $80 \mathrm{~K}$ and $250 \mathrm{~mA}$ dc bias current.

The response is calculated from both the analytic model and that from the basic RC-model. 

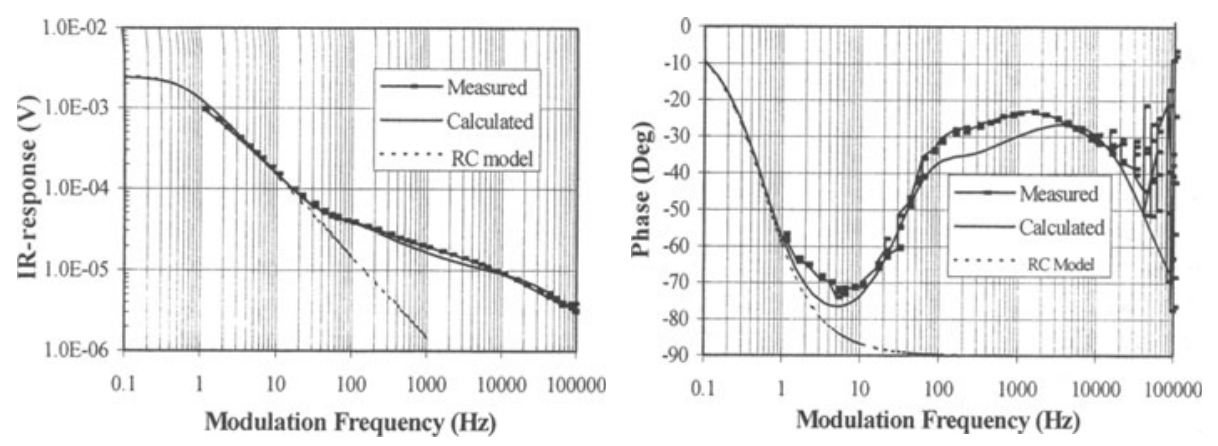

Figure 3.3. Measured and calculated magnitude (left) and phase (right) of response versus modulation frequency of the $\mathrm{LaAlO}_{3}$ substrate sample $061.02 \mathrm{a}$ at $80 \mathrm{~K}$ and $500 \mu \mathrm{A}$ bias current. The response is calculated from both the analytic model and that from the basic RC-model.

very high frequencies where the thermal diffusion length into the substrate or into the micromachined free standing base element of the bolometer becomes smaller or comparable to the thickness of the substrate or the base element [38]-[39]. In general the use of the basic $\mathrm{R}-\mathrm{C}$ model is appropriate in the frequency regimes where one period of the modulation frequency is longer than the low frequency time response of the samples, $\tau_{L}$. The $\tau_{L}$ is defined as $\tau_{L}=C(0) / G(0)$, where $G(0)$ and $C(0)$ are the total low or so called the dc thermal conductance and the heat capacity of the samples. In the following the results from the model to the measured values are compared and the effects of different thermal and dimensional parameters on the fit of the model to the experimental values are investigated. The comparison is done for both the magnitude and phase of the response in three regimes of low, mid, and high ranges of frequencies.

a) The Magnitude of Response at Low Frequencies and the Dependence on the Substrate Material and Dimensions

As given in Table 3.1, the substrate-holder thermal boundary resistance of the samples, $\mathrm{R}_{\mathrm{sc}}$, are given in per unit area and found using the measured dc thermal conductance of the samples using the shift in the temperature caused by Joule heating at elevated bias currents
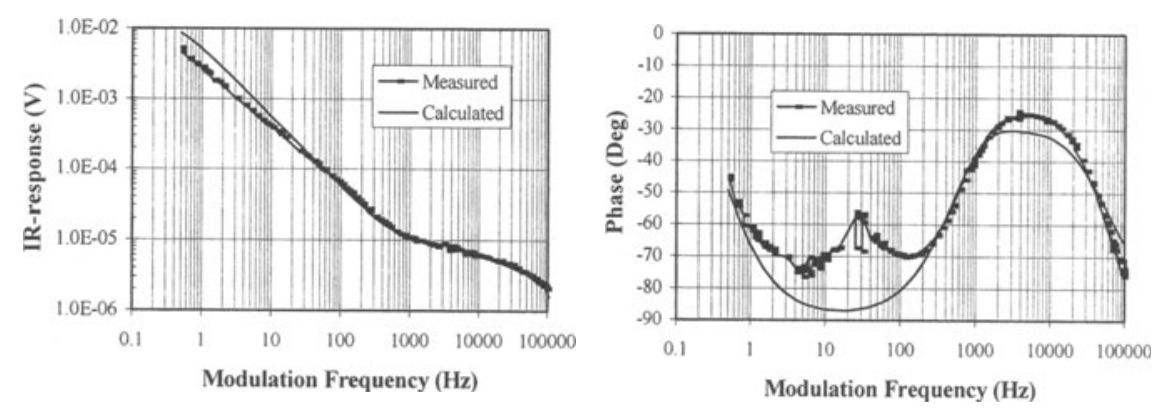

Figure 3.4. Measured and calculated magnitude (left) and phase (right) of response versus frequency of the $\mathrm{MgO}$ substrate sample number $064-03 \mathrm{a}$ at $79 \mathrm{~K}$ and $1 \mathrm{~mA}$ bias current. The response is calculated from both the analytic model and that from the basic RC-model. 
[24]. As introduced before the factor $\alpha$ is defined for the correction of $R_{s c}$ value and depends on the area of the pattern, A, the substrate thickness, $d_{s}$, and the thermal conductivity of the substrate materials, $k_{s}$. It increases by the increase of $k_{s}$ and the increase of the ratio of $d_{s} / A$ as expected. The requirement of the factor $\alpha$ for a better fit of the calculated response curves to the measured ones, is due to the spreading thermal resistance effect in the substrate material neglected by the one dimensionality of the model discussed here. Hence, for a relatively low thermal conductive substrate sample with a small $d_{s} / A$ ratio, this factor is expected to approach one as observed in table 3.1. Using the known parameters for the films and the substrates and the obtained values for the $\alpha$ and the boundary resistance', the phase and magnitude of the response for the samples are calculated and plotted in figures 3.2 to 3.4.

As shown in the figures, at low modulation frequencies there is a discrepancy between the results from the model and the measured values. This discrepancy increases by the increase of the thermal conductivity of the substrate materials showing its maximum for the most conductive substrate material. This is due to the one dimensionality of the model ignoring the lateral heat diffusion in the substrate materials underneath the pattern. By comparing the magnitude of the response curves in Figures 3.2, 3.3 and 3.4 for $\mathrm{SrTiO}_{3}, \mathrm{LaAlO}_{3}$, and $\mathrm{MgO}$ respectively, the discrepancy is the most for the most thermal conductive $\mathrm{MgO}$ substrate sample. This is also consistent with the variation of $\alpha$, which is the highest for the same sample. This discrepancy can be avoided by use of a three dimensional solution for the model, or use of an approximate cylindrical solution for a truncated cone structure with a linearly increasing base from the film to the bottom of the substrate. Such approximate solutions can also be applied using the simulation programs such as SPICE where the parameters can further be considered frequency dependent [7].

b) Phase of the Response and the High and low End Frequency Behavior

The phase of the response of the samples are much more sensitive to the values of the characteristic parameters of the samples. Particularly the drop of the phase at high-end frequency response has been the determining factor in finding the equivalent values of $\mathrm{C}_{\mathrm{f}}$ and $\mathrm{R}_{\mathrm{fs}}$ given in Table 3.1. While the low end calculated phase of the response is in a very good agreement with the experimental results for $\mathrm{SrTiO}_{3}$ and $\mathrm{LaAlO}_{3}$ substrate samples, there is a peak at the frequency range of 10 to $100 \mathrm{~Hz}$ in the $\mathrm{MgO}$ substrate sample, which cannot be explained by this model. This behavior has also been observed for all other $\mathrm{MgO}$ substrate samples. This occurs at lower frequencies in thinner substrate samples e.g. at about 10 to 20 $\mathrm{Hz}$ frequency for $0.025 \mathrm{~cm}$ thick $\mathrm{MgO}$ substrate samples compared to that of a $0.5 \mathrm{~cm}$ thick $\mathrm{MgO}$ substrate sample 064-03a shown in Figure 3.4b. One speculated mechanism for this is possibly due to the reflection and interference effect of the acoustical phonons within the boundaries of the substrate underneath the superconducting pattern [24]. The possible effect of the superconducting material on the spectral phonon density in the substrate and its consequent effects is discussed in next sections.

c) Mid-Range Frequency Behavior of the Phase and Magnitude of the Response

There is a slight discrepancy in the midrange frequency response curves compared to that of the model. This is clearly seen in the plateau of the phase versus frequency curves for all the samples shown in Figures3.2 to 3.4. The discrepancy is also seen partly in the magnitude of the response curves in the same frequency regime. The measured response in this range is slightly higher in magnitude, and more in phase with the radiation signal than the expected values from the model. 


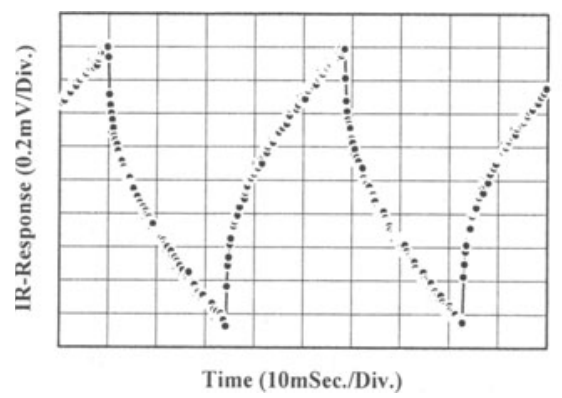

Figure 3.5. The steady state response versus time of the $\mathrm{SrTiO}_{3}$ substrate sample $064-01$ a at $560 \mu \mathrm{A}$ bias current, radiated by a $20 \mathrm{~Hz}$ square radiation signal with $2.13 \mathrm{~mW} / \mathrm{cm}^{2}$ maximum intensity.

The possible effect of the radiation absorption by the open areas of the substrate within the meander lines of the patterns has also been investigated as a possible source for the discrepancy. This consideration in the model Leeds to lower phase of the response increasing the observed discrepancy in the plateau region of the phase curves particularly for the $\mathrm{SrTiO}_{3}$ substrate sample. The spectral absorption of the crystalline substrate materials from $600 \mathrm{~nm}$ to $50 \mu \mathrm{m}$ are about $12 \%, 20 \%$, and $8 \%$ for the $0.05 \mathrm{~cm}$ thick $\mathrm{SrTiO}_{3}, \mathrm{LaAlO}_{3}$, and $\mathrm{MgO}$ substrates at $850 \mathrm{~nm}$ radiation wavelength respectively. While the effect of the substrate absorption is negligible in the response of the samples measured at $850 \mathrm{~nm}$ radiation wavelength, it has a major effect in the measured spectral response of the samples at longer wavelengths [7], [9], [11], [37]. The effect of the substrate absorption in the spectral response of the samples is studied in next sections.

A small and relatively fast component in the time response of the samples also is reported which relatively increases compared to the total response as the modulation frequency increases. This cannot be explained by the thermal response as modeled in this work. The steady state time response of $\mathrm{SrTiO}_{3}$ substrate sample 064-01a in the above mentioned frequency regime, is shown in Figure 3.5. As observed from the figure there exist an abrupt jump and drop at the edge of the on and off switching times of the radiation source. The source is a fast near IR source, which is derived by a square wave voltage signal. The same type of the steady state time response is observed for all other samples in the same frequency regime. The effect of this fast component of the response is in the favor of the discrepancy (i.e. lowering the phase value and increasing the magnitude of the response from those calculated values by the model). This is possibly the non-thermal component of the response and being a possible source for the discrepancy that needs to be further investigated.

\section{Response of thick film ETBs}

Thick film IR detectors are typically fabricated by screen-printing technology using pastes manufactured from high- $\mathrm{T}_{\mathrm{c}}$ superconducting powders on polycrystalline substrates such as $\mathrm{MgO}$ and $\mathrm{Al}_{2} \mathrm{O}_{3}$ [4]. The thickness of the superconducting films obtained by this method is in the range of a few 10 s of micrometers, being about $30 \mu \mathrm{m}$ for the studied samples here. The $\mathrm{Y}$-Ba-Cu-O compound with 123 and 124 stoichiometries can be used for fabrication of thick film superconductive infrared detectors using the hybrid technology. The fabricated films are then post annealed in various ambient such as Oxygen at high temperatures resulting granular 


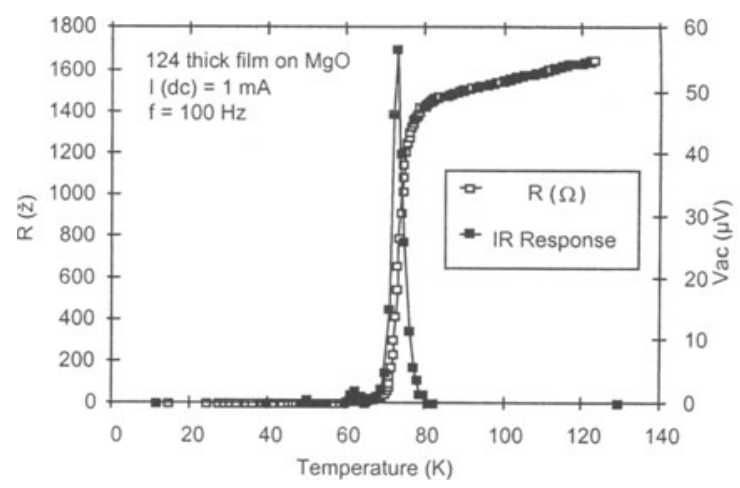

Figure 3.6. Resistance and magnitude of the response versus temperature of a $100 \mu \mathrm{m}$ wide and $4 \mathrm{~mm} \times 6 \mathrm{~mm}$ total area thick film YBCO on $\mathrm{MgO}$ substrate sample.

superconductive films with a 123 phase [29]. The thick film samples here are screen printed on polycrystalline $\mathrm{MgO}$ substrates. The film geometry are a serpentine pattern, with a line of $250 \mu \mathrm{m}$ width and $82.6 \mathrm{~mm}$ length, $0.89 \mathrm{~mm}$ pitch and $30-35 \mu \mathrm{m}$ in thickness [4].

At sufficiently high annealing temperatures, about $850^{\circ} \mathrm{C}$ in air, the detectors made of material with 124 phase of YBCO converts to the 123 phase plus a $\mathrm{CuO}$ species. The conversion of 124 material to $123+\mathrm{CuO}$ at high temperatures can give 123 material with higher critical currents [29], permitting a higher bias current for the bolometer. The $\mathrm{CuO}$ species, considered to be uniformly distributed in the bulk, can behave as pinning centers for fluxons and prevent flux flow in the material [24].

The IR-response versus temperature measurement on a 124-based sample (converted to 123 phase) is shown in Figure 3.6. The results of the IR-response vs. temperature measurement are superimposed on its $\mathrm{R}$ vs. $\mathrm{T}$ transition curve in the figure. It should be noted that there is a small peak at $60 \mathrm{~K}$ to $65 \mathrm{~K}$ in the response, which is below the region of its transition-temperature curve $\left(\mathrm{T}<\mathrm{T}_{\mathrm{c}-\text { zero }}\right)$. A similar feature is not reported in the IR-response of the sample at lower dc-bias current. This response, which shows a dependence on the dc-bias current through the sample, is attributed to a Kosterlitz-Thouless phase transition and the associated noise signal below $\sim T_{c}$ as discussed in section 7 .

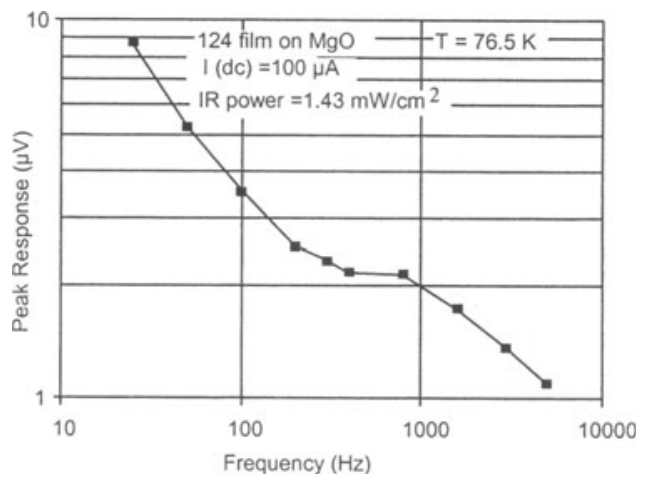

Figure 3.7. Magnitude of the response versus frequency of a $100 \mu \mathrm{m}$ wide and $4 \mathrm{~mm} \times 6 \mathrm{~mm}$ total area thick film YBCO on polycrystalline $\mathrm{MgO}$ substrate sample. 
An IR-response vs. frequency measurement on a thick film is shown in Figure 3.7. As shown in the figure, there are two breakpoints in the response. The first breakpoint, near 400 $\mathrm{Hz}$, is attributed to the thermal time response, $\tau(\mathrm{C} / \mathrm{K})$, of the substrate and matches the preceding analysis with respect to the effect of the substrate-holder thermal boundary resistance. The second breakpoint, near $800 \mathrm{~Hz}$, is attributed to the thermal time response of the thick superconducting film with the consideration of the film-substrate boundary resistance. Considering this assumption, the second breakpoint is expected at much higher chopping frequency for thinner films as discussed earlier.

\section{THE TEMPERATURE DEPENDENCE OF RESPONSIVITY AND DEVIATIONS FROM THE DR/DT CURVE}

The phase and amplitude of the response to infrared signals of edge transition bolometers versus temperature is studied in this section. The measured amplitude and the phase of the response versus temperature of the devices deviate from that expected based on the discussed models in the preceding sections. In this regard a model is discussed here that can explain the observed discrepancy in the response versus temperature from the $\mathrm{dR} / \mathrm{dT}$ curve in the studies samples in this chapter and some of other reported discrepancies from the bolometric response in the literature [22]-[23], [45]. Based on this model, the thermal constants, G, and C, change as the film goes into the superconducting state; $G$ becomes strongly temperature dependent, and decreases with decreasing temperature. A probable cause for the change in $\mathrm{G}$ is a shift in the nature of the phonons emitted by the superconductor as it goes through $\mathrm{T}_{\mathrm{C}}$.

There is a dependence of the measured total dc thermal conductance, $G(0)$, on the thickness of the substrate of the samples. Based on the preceding analysis, $G(0)$ is always limited by the substrate/cold-head thermal boundary resistance at low modulation frequencies [7], [18]. This conclusion is confirmed by comparing the results to a model of the device's frequency response as discussed earlier. Also using the low frequency response of the bolometers, the specific heat and thermal conductance of the substrate material can be verified to confirm the a above approach [7], [18]. The study of this part has been focused on the characteristics of $\mathrm{YBCO}$ films on $\mathrm{MgO}$ substrates samples in response to infrared radiation with a wavelength of $\lambda=0.85 \mathrm{~nm}$ emitted from a near IR-LED source.

\section{A. Thermal conductance characterization}

As discussed earlier, the thermal conductance of a bolometer is defined as the total heat conduction from the bolometer to its environment per degree of temperature difference. The amount of input power (heat), $P_{\text {in }}$, to a bolometer can be determined, and used to measure $G$ from:

$\mathrm{G}=\frac{\mathrm{P}_{\text {in }}}{\Delta \mathrm{T}}$,

where $\Delta \mathrm{T}$ is the difference between the temperature of the bolometer and its environment. The thermal conductance of a superconductor bolometer can be measured in different ways, which are discussed here in more details. In each technique there are different kinds of possible error associated with the nature of the characterization [7], [24]. Heat loss by radiation from the superconducting bolometer (superconducting film and the substrate) in typical systems is normally negligible compared to the phonon heat conduction [7], [30]. 
One of the effects of the finite thermal conductance in a sample can be observed in the $\mathrm{R}$ versus $T$ curves at different bias currents. The thermal conductance of the sample puts a limit on the maximum bias current that can be used in the measurement. The bias current at any temperature higher than $\mathrm{T}_{\mathrm{c} \text {-zero }}$, is limited by thermal runaway, characterized by the factor $\alpha$ defined as [1]:

$\alpha=\frac{\mathrm{I}^{2} \mathrm{dR}}{\mathrm{G}(0) \mathrm{dT}} ; \quad$ with $\alpha<1$ required for stability,

where $I$ is dc bias current and $G(0)$ is the dc thermal conductance. The effect of instability in the temperature of the film, due to high bias currents, will appear as an unrealistically sharp transition in the $R$ versus $T$ curve [25], [42]. The instability and the shift in the $T_{c \text {-onset }}$ can be used to measure $\mathrm{G}(0)$.

At low bias currents the temperature of the superconducting film is very close to the temperature of the cold-head. As the bias current increases, a temperature gradient develops across the substrate and the interfaces due to Joule heating in the film. Since it is the temperature of the holder which is commonly controlled, this results in a shift of the bias point in the superconducting film towards $\mathrm{T}_{\mathrm{c} \text {-onset }}$ where $\mathrm{dR} / \mathrm{dT}$ has lower values. The decrease of $\mathrm{dR} / \mathrm{dT}$ lowers the detector response [25], which has been interpreted as the effect of the bias current on the intrinsic part of the response by some groups [22]. The drop of the response at high currents can be used as a different method to measure $\mathrm{G}$. Knowing the $\mathrm{dR} / \mathrm{dT}$ versus $\mathrm{T}$ curve, and the change in the response, the shift in the temperature of the superconducting film with respect to the holder can be found. Using the $\mathrm{R}$ and the bias current, the power dissipation in the film can be obtained. Then by use of the temperature shift and the dissipated Joule power in equation (4.1), the value of $\mathrm{G}(0)$ can be calculated [24].

The most convenient way to obtain $\mathrm{G}(0)$ is to make use of the slope of the R versus $T$ of the films in the normal state near $T_{c}$ as shown for sample 064-02b in Figure 4.1. At high currents, $\mathrm{R}$ increases due to the $\mathrm{dc}$ power dissipation heating the film. The relationship between the increase in the resistance of the film, $\mathrm{R}$, and the bias current due to Joule heating is:

$\Delta \mathrm{R}=\mathrm{I}^{2} \mathrm{R} \frac{\mathrm{dR}}{\mathrm{GdT}}$.

Using the measured values for the $\mathrm{dR} / \mathrm{dT}$ at the bias temperature of the holder, and the bias currents, values of $\mathrm{G}$ can be obtained for each data point. There is a slight error in the above

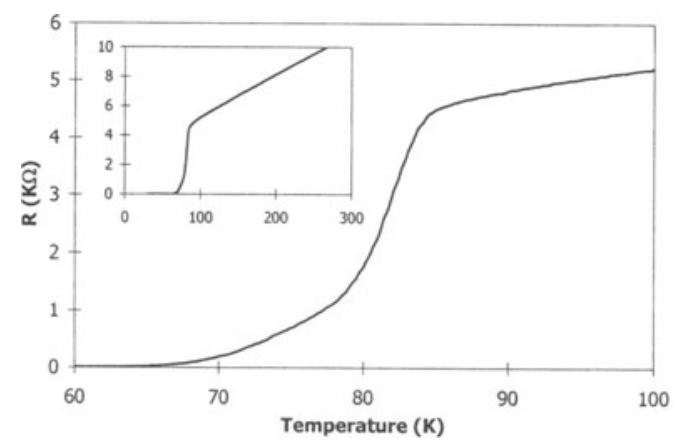

Figure 4.1. $\mathrm{R}$ versus temperature of sample $064-02 \mathrm{~b}$ at $100 \mu \mathrm{A}$ bias current and 2 $\mathrm{K} / \mathrm{min}$ heating rate. Insert shows full temperature range. 
method due to the change of the $\mathrm{dR} / \mathrm{dT}$ at different temperatures.

Here one can use a method, which eliminates the above error. Considering Joule heating by the bias currents as the source for the shift of the film temperature, $T_{f}$, with respect to the temperature of the holder, $\mathrm{G}(0)$ can be found from:

$\mathrm{G}(0)=\frac{I^{2} \mathrm{R}}{\Delta \mathrm{T}}$

where $\Delta \mathrm{T}$ is the difference between $\mathrm{T}_{\mathrm{f}}$ and the temperature of the cold-head. The resistance of the film, $\mathrm{R}$, can be measured from $\mathrm{V} / \mathrm{I}$ at each point. Using the $\mathrm{R}$ versus $\mathrm{T}$ curve for the region of interest $(90<T<120 \mathrm{~K})$, and the measured value of $R$ at different bias currents $T_{f}$ can be obtained and used to determine $\Delta \mathrm{T}$. Then using $\Delta \mathrm{T}$ and $\mathrm{P}_{\mathrm{in}}=\mathrm{I}^{2} \mathrm{R}$ in equation (4.4), values of $\mathrm{G}(0)$ can be obtained for each data point. This is the method used to find the averaged values given in III_Table 2.1. To measure $\mathrm{G}(0)$ relatively high bias currents should be used for ease in monitoring the temperature change in the film. However, high currents through the material can affect the electrical properties of the films shifting $T_{c}$ to lower temperatures [25], [48].

\section{B. Dependence of $\mathrm{G}$ on the YBCO film pattern and the substrate thickness}

The values obtained for the dc or low frequency thermal conductance of the studied bolometers are about 2-3 orders of magnitudes lower than the values calculated from the properties of the substrate material, which can be obtained from:

$\mathrm{G}=\mathrm{k} \frac{\mathrm{A}}{\mathrm{L}}$.

Where $\mathrm{k}$ is the thermal conductivity of the substrate material and L and A are thickness of the substrate and area of the superconducting pattern. The substrate/cold-head thermal boundary resistance determines the measured G values in III_Table 2.1 [7], [25]. This can be found from the frequency response of the devices. At low frequencies, the thermal diffusion length into the substrate, $L_{f}[2],[7],[18],[60]$, is larger than the substrate thickness, and $G$ is constant with respect to $f$, and much lower than the values calculated for the substrate from equation (4.5). As the frequency increases, $L_{f}$ becomes comparable or smaller than the substrate thickness changing $\mathrm{G}$, which changes the slope of the response versus modulation frequency from a $f^{-1}$ to $\mathrm{a} \mathrm{f}^{0.5}$ dependence [2], [7], [16]. Then $\mathrm{G}$ is limited by the thermal conductance of the substrate material, and can be obtained from equation (4.5), where $L$ is proportional to the thermal diffusion length, $\mathrm{L}_{\mathrm{f}}[7]$, [18], [24].

The measured thermal boundary resistance at the substrate/cold-head interface can be explained by the thermal acoustic mismatch theory or Kapitza resistance [16], [30]. A thermal boundary resistance defined as the Kapitza resistance, is:

$\mathrm{R}_{\mathrm{k}}=\frac{\Delta \mathrm{T}}{\mathrm{Q}^{\prime}}$

where $Q^{\prime}$ is the heat flux per unit area through the interface, and $\Delta \mathrm{T}$ is the temperature difference across the boundary. This phenomenon is related to the reflection and refraction of the incident thermal phonons at the interface [30]. Based on the thermal acoustic mismatch model, $R_{k}$ is temperature dependent with a dependence of $T^{-3}$ [24], [30]. In the model, it is assumed that the heat is carried only by thermal phonons (only acoustic phonons are important at low temperatures), the two solids are perfect materials, and finally the interface is perfect or ideal [94], [95]. Based on the above assumptions the heat transfer through the interface is 
determined mainly by the acoustic impedance $\rho_{\mathrm{m} 1} S_{1}$ and $\rho_{\mathrm{m} 2} S_{2}$ of the two attached materials at the boundary, where $\rho$ is the mass density and $S$ is the velocity of sound in the material.

As shown in Figure 2.1, there are two interfaces to be considered in our experimental configuration. The film/substrate interface and the substrate/cold-head interface. The film/substrate interface and its corresponding thermal resistance, $R_{b d}$, have been investigated by different groups [15], [17], [30]. The effect of the $R_{b d}$ on the response in the samples of table 2.1 is only at high frequencies, at which the thermal resistance of the substrate becomes comparable to the film/substrate thermal boundary resistance. This frequency is $53 \mathrm{KHz}$ for $0.05 \mathrm{~cm}$ thick $\mathrm{MgO}$ substrate sample [4], [7], which is obtained from a typical value of $\mathrm{R}_{\mathrm{bd}}=1.1 * 10^{-3} \mathrm{~K}-\mathrm{cm}^{2} / \mathrm{W}$ [4], [15], [17]. Though $\mathrm{G}$ is limited by the substrate/cold-head thermal boundary resistance, $\mathrm{R}_{\mathrm{s}-\mathrm{c}}$, at modulation frequencies less than $720 \mathrm{~Hz}$, and $2.8 \mathrm{KHz}$, for 0.05 $\mathrm{cm}$ and $0.025 \mathrm{~cm}$ thick $\mathrm{MgO}$ substrate samples, respectively [4], [7].

However, a dependence of $\mathrm{G}(0)$ on the substrate thickness and the pattern area exists as given in Table 2.1. It should also be noted that the back of same substrates cannot be thoroughly cleaned due to the adhesion of silver epoxy applied to the back of the substrate for the in-situ deposition of the YBCO and this can cause a slight difference in the measured $\mathrm{G}$ compared to the $\mathrm{G}$ of a sample with a perfectly clean and smooth contact to the cold-head.

As given in Table 2.1, G(0) increases for thinner substrates, while it is still limited by the substrate/cold-head thermal boundary resistance. The dependence of the value of $G(0)$ on substrate thickness can be attributed to scattering of phonons within the substrate which changes the angular distribution of the phonons at the substrate/cold-head interface. Phonons with a larger angle of incidence to the normal at the interface will be internally reflected at the boundary, lowering the value of $\mathrm{G}(0)$. This is also consistent with the observed dependence of the $\mathrm{G}(0)$ on the area of the superconducting pattern, it increases with an increase in the area of the pattern, suggesting that the conduction is mainly through the contact area below the pattern with direct on-line flow to the cold-head.

\section{Effect of bias current on the response and $d R / d T$ versus temperature}

The shift of the temperature of the film with respect to the temperature of the cold-head at high bias currents, used to measure $G(0)$ ), can also be observed in the bolometric response versus $T$ of the samples. The temperature of the peak in the response versus $T$ curve shifts to

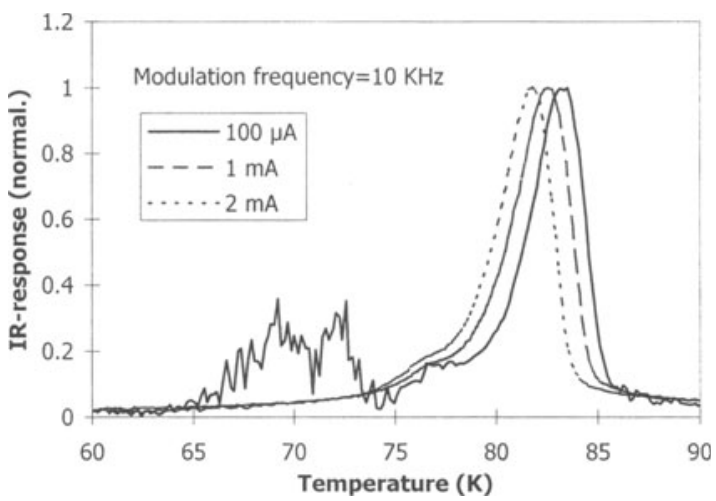

Figure 4.2. Normalized response of sample $064-02 \mathrm{~b}$, at $100 \mu \mathrm{A}, 1 \mathrm{~mA}$, and $2 \mathrm{~mA}$ bias currents with peak response of $386 \mathrm{nV}, 3.28 \mu \mathrm{V}$, and $5.87 \mu \mathrm{V}$, respectively. 
lower temperatures as higher bias currents are used. Though the response versus temperature will follow the measured $R$ versus $T$ curve (or the corresponding $\mathrm{dR} / \mathrm{dT}$ curve), the magnitude of the response follows the real slope of the transition, the $R$ versus temperature of the film (the slope at low bias currents) [7]. This effect for sample 64-02b is shown in Figure 4.2. The values of thermal conductance obtained by the shift in the peak of the response versus $T$ of the samples, confirm the range of values given in Table 2.1. A slight change in $G$ is expected, due to the difference of the temperature at the peak of the IR-response and the temperature at which the $G$ values in Table 2.1 are measured. Also there is an error associated with the above method due to the continuous change of the temperature during the measurements [4].

As shown in Figure 4.2, there is a small peak at temperatures below the $\mathrm{T}_{\text {c-zero }}$ for the response at $100 \mu \mathrm{A}$ bias current, which is attributed to be the intrinsic noise of the sample. This excessive noise is in all samples that have weak links, or are granular and show a long tail in their $R$ versus $T$ curves. The peak noise in this region decreases with increasing bias current, while it widens on the lower temperature end. A detailed study of the different types of noise in the samples is given in later section.

\section{Response versus temperature of the ETBs and deviations from the thermal model}

Assuming the thermal parameters $\mathrm{G}$ and $\mathrm{C}$ to be independent of temperature as given in equation (2.1), the bolometric response is expected to follow the $\mathrm{dR} / \mathrm{dT}$ versus $\mathrm{T}$ curve. Figure 4.3 shows the magnitude of the IR-response of sample $064-02 \mathrm{~b}$ to a $20 \mathrm{~Hz}$ modulation frequency, and the corresponding $\mathrm{dR} / \mathrm{dT}$ versus $\mathrm{T}$ curve. Both curves are normalized to their values at the $\mathrm{T}_{\mathrm{c} \text {-onset }}$. The $\mathrm{dR} / \mathrm{dT}$ of the sample was obtained by use of the simultaneously measured $R$ versus $T$. As observed in Figure 4.3, there is a slight discrepancy between the response and the $\mathrm{dR} / \mathrm{dT}$ curve at the lower part of the transition region closer to $\mathrm{T}_{\mathrm{c} \text {-zero. }}$. There have been several reports on such discrepancies [45]. The discrepancy in these samples is dependent on the modulation frequency of the signals, decreasing as the frequency increases and is smaller for samples with sharp transitions. This is verified for the frequency range of 20 $\mathrm{Hz}$ to $10 \mathrm{KHz}$ [4].

The phase of the response of the samples also varies as a function of temperature. This variation indicates a change in the thermal constants of the bolometer. The measured phase of the response versus temperature of sample $064-02 \mathrm{~b}$ at $20 \mathrm{~Hz}$ frequency, is given in Figure 4.4.

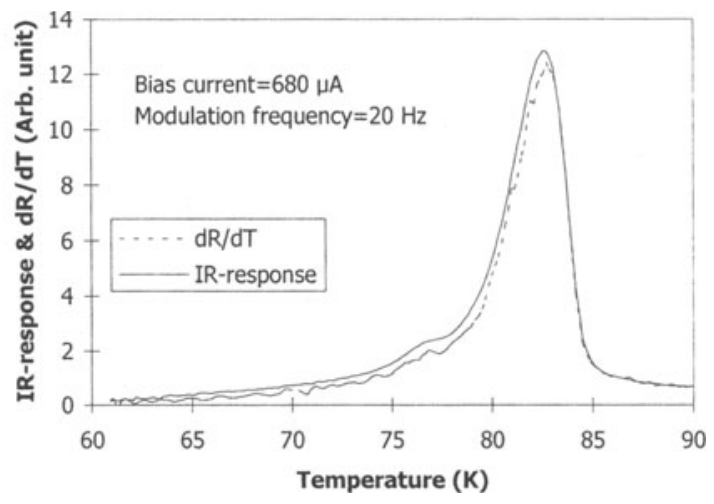

Figure 4.3. Normalized $I R$ response and $d R / d T$ vs. temperature of the sample $064-02 \mathrm{~b}$ at $20 \mathrm{~Hz}$ and $680 \mu \mathrm{A}$ bias current. 


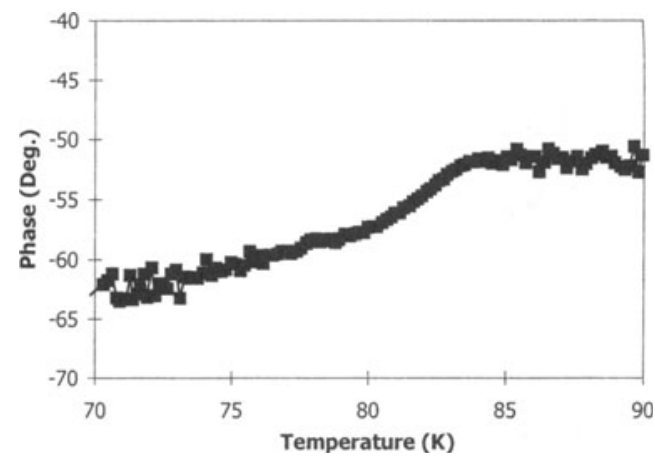

Figure 4.4. The phase of the IR-response vs. temperature of sample $064.02 \mathrm{~b}$ at $20 \mathrm{~Hz}$ modulation frequency and $680 \mu \mathrm{A} \mathrm{dc}$ bias current.

The response at this frequency is mainly bolometric [7], in agreement with the response versus time of the sample [4]. The variation of the phase versus temperature in Figure 4.4 shows a dependence on the superconducting transition of the film. The magnitude of the phase of the response of the samples increases at the lower part of the transition. This variation of the phase versus temperature, which follows the superconducting transition, suggests a correlation between the thermal characteristics of the superconducting film and the response at low frequencies. It is already discussed that the response at low modulation frequencies is mainly governed by the thermal constants of the substrate material and substrate/cold-head interface i.e. $\tau=\mathrm{C} / \mathrm{G}$ with $\mathrm{C}$ determined from the heat capacity of the substrate and $\mathrm{G}$ the substrate/coldhead interface conductance. The same type of behavior of the phase of the response is observed for the other samples as well, and for samples with sharper transitions, the change is smaller [4]. One explanation for the change in the response is that the character of the phonons changes as the film goes into the superconducting state. In the normal state one expects the thermal phonons to be from the acoustic branch. In the superconducting state the IR-photons can break pairs, which upon recombination emit phonons that have energy of $2 \Delta(T)$. These phonons have a different distribution than the thermal ones in the acoustic branches between longitudinal and transverse phonons, and may also include optical phonons. This is discussed in detail in the following section.

The increase of the amplitude of the response at the lower part of the superconducting transition at low frequencies, suggests a decrease in $G$, assuming that the heat capacity contributing to the response is a constant. From equation (2.1), a decrease in G should give an increase of the phase (lag), which is consistent with the measured increase of the magnitude of the phase given in Figure 4.4. The discrepancy in the magnitude of the response versus temperature of sample No. 064-02b is given in Figure 4.5. This shows that the deviation increases as the temperature drops. The variations in the ratio are a measure of the noise present. The measurements at lower temperatures are limited by the small signal, which decreases the signal to noise ratio.

\section{E. Effect of variation of $G$ on the response and its frequency dependence}

Considering the discrepancy at $20 \mathrm{~Hz}$ in Figure 4.5, the response at $70 \mathrm{~K}$ is about 1.4 times higher than the expected values. This temperature is close to $\mathrm{T}_{\mathrm{c}-\mathrm{zero}}$ and will be noted as 


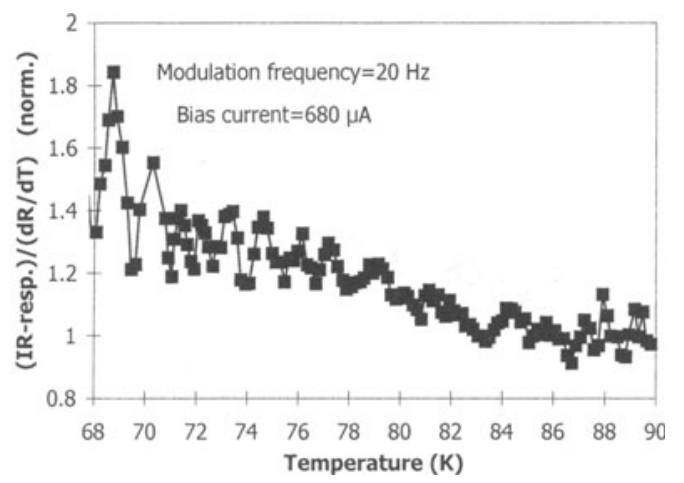

Figure 4.5. Deviation of IR-response vs. temperature from the $\mathrm{dR} / \mathrm{dT}$ vs. temperature in Figure 4.4.

$\mathrm{T}_{\mathrm{c}-\mathrm{low}}$. From Figure 4.4 , the phase of the response, $\theta$, at the onset temperature of $\sim 85 \mathrm{~K}$ is about $-51.5^{\circ}$ and at the $\mathrm{T}_{\text {c-low }}(\sim 70 \mathrm{~K})$ is about $-62.5^{\circ}$. Then equation $(2.1)$ results in:

$-\tan \theta_{\mathrm{i}}=\omega \tau_{\mathrm{i}}$

where $\theta_{\mathrm{i}}$ is the temperature dependent phase of the response. Then:

$\omega \tau_{\text {c-onset }}=1.26=\omega \mathrm{C}_{\mathrm{c} \text {-onset }} / \mathrm{G}_{\mathrm{c} \text {-onset }}$

Similarly for the phase at $\mathrm{T}_{\mathrm{c}-\text { low }}$ we have:

$\omega \tau_{\text {c-low }}=1.92=\omega \mathrm{C}_{\mathrm{c} \text {-low }} / \mathrm{G}_{\mathrm{c} \text {-low }}$

Now, based on the above analysis, from the ratio of equation (4.8) and (4.9) one gets:

$\left(\tau_{\text {c-onset }} / \tau_{\mathrm{c}-\text { low }}\right)=0.65$

or:

$\frac{\mathrm{G}_{\text {c-onset }} \mathrm{C}_{\text {c-low }}}{\mathrm{G}_{\text {c-low }} \mathrm{C}_{\text {c-onset }}}=1.53$

Assuming the heat capacity of the bolometer, which is mainly due to the substrate material at this frequency, drops from $\mathrm{T}_{\mathrm{c} \text {-onset }}$ to $\mathrm{T}_{\mathrm{c}-\mathrm{zero}}$ according to its experimental results [61], inquires that the thermal resistance of the bolometer increases ( $G$ decreases) at lower temperatures, and consequently there would be an increase in the response which is consistent with the result in Figure 4.5. The variation in the responsivity, taking the changes in the $\mathrm{C}$ into account, can be obtained by use of equation (2.1) from:

$\frac{r_{v}(c-l o w)}{r_{v}(c \text {-onset })}=\frac{G_{c-o n s e t} \sqrt{1+\omega^{2} \tau_{c-\text { onset }}^{2}}}{G_{\text {c-low }} \sqrt{1+\omega^{2} \tau^{2}{ }_{c-l o w}}}$

By use of the results from Figure 4.3, and values in equation (4.8) and (4.9) in equation (4.12), a value of 1.89 can be obtained for $\mathrm{G}_{\mathrm{c}-\text { onset }} / \mathrm{G}_{\mathrm{c}-\text { low }}$. By applying this to equation (4.11), a value of 0.81 is obtained for $\mathrm{C}_{\mathrm{c} \text {-low }} / \mathrm{C}_{\mathrm{c} \text {-onset }}$ ratio. The change in the $\mathrm{C}$ can be attributed to the change of the effective heat capacity due to the change in the characteristics of the phonons and the their propagation and also the temperature dependence of the heat capacity of the substrate material as expected for a crystalline solid.

The thermal conductance at low frequencies is determined by the substrate/cold-head thermal boundary resistance, which can be strongly temperature dependent [24]. Based on 
above analysis, as the temperature decreases the substrate/cold-head thermal boundary resistance increases as expected for a solid/solid interface [24]. However, the phase of the response shows its strongest variation as the sample goes through the superconducting transition, indicating that these large changes are associated with differences between the normal and superconducting state, rather than the temperature dependence of the interface boundary resistance as modeled in the next section. At higher modulation frequencies, the thermal diffusion length into the substrate material becomes comparable to or smaller than the substrate thickness. Then the thermal conductance becomes independent of the substrate/coldhead interface, and is expected to be mainly governed by the thermal conductivity of the substrate material, $\mathrm{k}_{\mathbf{s}}$. The frequency at which the thermal diffusion length becomes equal to the substrate thickness of sample $064-02 \mathrm{~b}$ is $2.8 \mathrm{kHz}$ [4], [7]. For frequencies higher than this the $\mathrm{G}$ is governed by the $\mathrm{k}_{\mathrm{s}}$ [4], [7]. The thermal conductivity of the substrate material can increase as the temperature decreases. This can compensate for the decrease in the $\mathrm{C}$, reducing the discrepancy, and at higher frequencies it may even cause a decrease in the response at the $\mathrm{T}_{\mathrm{c}-\text { low }}$ compared to the response at the $\mathrm{T}_{\mathrm{c} \text {-onset. }}$. Hence the response at higher frequencies can be closer to the $\mathrm{dR} / \mathrm{dT}$ curve discussed in the following section.

\section{EFFECTS OF SUPERCONDUCTIVITY TRANSITION AND THERMAL PARAMETERS ON THE PHASES OF THE RESPONSE}

According to equation (2.1) the temperature-dependent phase of the response can be calculated by the formula, $\tan \theta=-\omega C / G$.

It is important to understand the factors that determine $G$ and $C$ of the bolometers as a function of the temperature, frequency, and other parameters of the bolometer configuration. The phase of the response to infrared signal of the edge-transition bolometers versus temperature at high and low modulation frequencies is studied in this section. A model is discussed that can explain the observed discrepancy in the response versus temperature discussed in previous section. According to the model, the thermal constants, $G$ and $C$, change as a film goes into the superconducting state. This might also partly be accounted as for some observed non-bolometric component of the response in this type of bolometers.

As shown in Figure 5.1, there are two thermal boundary resistances at the substrate interfaces, one at the film interface and the other at the holder interface. There are also two major bulk areas, which constitute the overall heat capacity of the samples. One is due to the superconducting film, and one is due to the substrate material. In analyzing the different operation regimes of the edge-transition bolometer parameters, the frequency dependence of the thermal diffusion length also plays an important role as discussed earlier.

The phase of the response of the samples is more sensitive to the values of the characteristic parameters of the bolometers than the magnitude of the response. The phase of the response shows a strong variation as the sample goes through the normal-superconducting transition both at low and high modulation frequencies showing also modulation frequency dependence. At low frequencies, the phase of the response starts to decrease as the temperature decreases below about the onset temperature of the normal to superconducting transition (i.e. at $\mathrm{T}<\mathrm{T}_{\mathrm{c} \text {-onset }}$ ). Opposite behavior exists at high modulation frequencies, i.e. the phase of the response starts to decrease at $\mathrm{T}<\mathrm{T}_{\mathrm{c}-\mathrm{onset}}$. This clearly indicates that, large changes in the phase and magnitude of response are mainly associated with differences in the conduction mechanisms in the normal and superconducting states, rather than the well known 


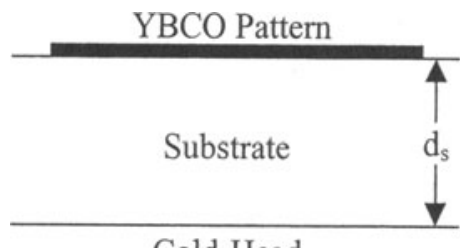

Figure 5.1. Lumped film sample configuration in contact with a cold-head. $d_{s}$ is the substrate thickness.

temperature dependence of the interface boundary resistance. The mechanisms causing the above behaviors are discussed in the followings.

\section{A. Low frequency regime}

At low modulation frequencies the phase of the response versus temperature increases by decrease of the temperature starting at $\mathrm{T}<\mathrm{T}_{\mathrm{c}-\text { onset }}$ as shown in the Figure 4.4. The respective amplitude of the response normalized to the measured $\mathrm{dR} / \mathrm{dT}$ of the superconducting film at $\mathrm{T}<\mathrm{T}_{\mathrm{c} \text {-onset }}$ for low modulation frequencies is shown in Figure 4.3. The amplitude of the response in Figure 4.3 increases above the expected value determined by the $\mathrm{dR} / \mathrm{dT}$ of the film as the temperature is lowered toward the $\mathrm{T}_{\text {c-zero }}$. Such behavior would be consistent with a decrease of the thermal conductance of the bolometer. The variation of the heat capacity at low temperatures, reveals typical $T^{3}$ dependence behavior [61]. At low modulation frequencies $(\omega=20 \mathrm{~Hz})$, the thermal conductance in the studied samples is determined by the substrate/cold-head interface conductance, or so called Kapitza boundary resistance, caused by the acoustic mismatch impedances of the interfaced materials [9], [18]. According to equation (2.17), by lowering the frequency the thermal diffusivity length into the substrate becomes longer than the substrate thickness and phonons emitted by the superconducting film into the substrate will reach substrate/cold-heat boundary.

The thermal boundary conductance at the substrate/cold-head interface can be explained by the theory of Kapitza conductance. It is well known [62], that Kapitza conductance is the derivative of the net heat flux transmitted across an interface with respect to the temperature difference between the two materials. Therefore, if one considers an interface between materials $\mathrm{A}$ and $\mathrm{B}$, we can write $G_{K}$ as follows:

$$
\begin{aligned}
\mathrm{G}_{\mathrm{K}} & =\frac{1}{\mathrm{~V}} \frac{\partial}{\partial \mathrm{T}} \sum_{\mathrm{k}} \sum_{\mathrm{j}}^{\mathrm{A}} \hbar \omega_{\mathrm{kj}} \mathrm{n}\left(\omega_{\mathrm{kj}}, \mathrm{T}\right) \mathrm{v}_{\mathrm{kjz}} \mathrm{t}_{\mathrm{kj}} \\
& =\frac{1}{\mathrm{~V}} \frac{\partial}{\partial \mathrm{T}} \sum_{\mathrm{k}} \sum_{\mathrm{j}}^{\mathrm{B}} \hbar \omega_{\mathrm{kj}} \mathrm{n}\left(\omega_{\mathrm{kj}}, \mathrm{T}\right) \mathrm{v}_{\mathrm{kjz}} \mathrm{t}_{\mathrm{kj}}
\end{aligned}
$$

Where the labels $\mathrm{A}$ and $\mathrm{B}$ on the sums indicate that all quantities in each sum correspond to phonons incident on the interface from the materials $A$ and $B$ sides, respectively. In equations (5.2) and (5.3), $\omega_{k j}$ is the phonon frequency, $\mathrm{v}_{\mathrm{kjz}}$ is the component of the phonon group velocity normal to the interface, $n\left(\omega_{k j}, T\right)$ is the Bose-Einstein distribution function, $\mathrm{t}_{\mathrm{Kj}}$ is the probability that a phonon of wave vector $\mathbf{k}$ and polarization $\mathrm{j}$ will be transmitted across the interface between materials $\mathrm{A}$ and $\mathrm{B}$, and $V$ is the volume. The Kapitza conductance can be 
calculated at low temperatures $\left(\hbar \omega_{D} / k T \gg>1\right)$ analytically, taking into account Debye phonon density of states, which can be obtained from;

$D(\omega)=V^{-1} \sum_{k j} \delta\left(\omega-\omega_{k j}\right)=\omega^{2} / 2 \pi^{2} c^{3}$,

where $c$ is the velocity of sound in the material. As a result from calculations, the thermal conductance has $T^{3}$ characteristics at low temperatures, and remains constant at high temperatures. The phonon system in each material in above calculations is considered to be at the equilibrium nature. However, in the calculation of the boundary conductance of the substrate/cold-head interface, one should take into account the phonon density of state spectrum of the non-equilibrium phonons, which propagates into the $M g O$ substrate and reach boundary substrate/cold-head (diffusion regime of propagation of phonons). Hence should also be determined the differences in the photo-absorption process in normal and superconducting states should also be determinded. As shown by the analysis of thermalization and photo-absorption in $\mathrm{YBaCuO}$ superconductors [63], interaction between hot quasi-particles and Cooper pairs condensate continues to divide the excess energy by forming three quasi-particles. The hot quasi-particles continue to break additional pairs by the electron-electron interaction process as they thermalize toward the energy gap by discrete energy steps. As a result there exists a characteristic feature- a jump at frequency $\omega=2 \Delta(T)$ due to activation of the recombination mechanism of phonon generation [64] (for $\omega \leq 2 \Delta(T)$ phonons are generated as a result of the energy relaxation of high energy quasi-particles). The phonon distribution function (product of $D(\omega) n(\omega, T)$ ) has fairly narrow maximum near $\omega=2 \Delta(T)$ [50], i.e. in the above calculation one should replace Debye phonon density of states by the Einstein phonon density function;

$D(\omega)=\omega^{2} \delta(\omega-2 \Delta(T))$.

Assuming that transmission probability and normal component of the group velocity to the interface to be independent of temperature, equation (5.2) can be rewritten as;

$\mathrm{G}_{\mathrm{K}}=\frac{1}{\mathrm{~V}} \sum_{\mathrm{j}} \int_{0}^{\omega_{\text {Debye }}} \mathrm{d} \omega \hbar \omega_{\mathrm{kj}} \mathrm{D}(\omega) \frac{\mathrm{dn}\left(\omega_{\mathrm{kj}}, \mathrm{T}\right)}{\mathrm{dT}} \mathrm{v}_{\mathrm{kjz}} \mathrm{t}_{\mathrm{kj}}$,

where for the $\operatorname{dn}(\omega, T) / d T$ we have the following expressions:

$\frac{\mathrm{dn}(\omega, \mathrm{T})}{\mathrm{dT}}=\left\{\begin{array}{l}\frac{\mathrm{k}}{\hbar \omega}, \frac{\hbar \omega}{\mathrm{kT}}<<1 \\ \frac{\mathrm{k}}{\hbar \omega} \mathrm{x}^{2} \exp (-\mathrm{x}), \mathrm{x}=\frac{\hbar \omega}{\mathrm{kT}}>>1\end{array}\right.$

It is clear that at high phonon frequencies, $2 \Delta(T)$ is about equilibrium temperature of the substrate $T$, and the contribution of the non-equilibrium phonons to the heat transfer has exponential behavior. As shown by the above calculations, at temperatures close to critical temperature $2 \Delta(T)<<T$, where $\Delta(T)=\Delta_{0}\left(1-T / T_{c}\right)^{1 / 2}$ (standard BCS theory for example [65], low frequency phonons do not influence heat transfer process and boundary conductance at substrate/cold-head. Hence calculated normalized conductance based on the equation (5.5), (5.6), and (5.7) are in good agreement with experimental data for the thermal conductance versus temperature at low modulation frequency. The discrepancy between experimental data and calculations at temperatures close to $T_{c}$ shown in_Figure 5.2, is associated with the tendency of the emitting phonon frequency to zero. Here we also consider superconducting 


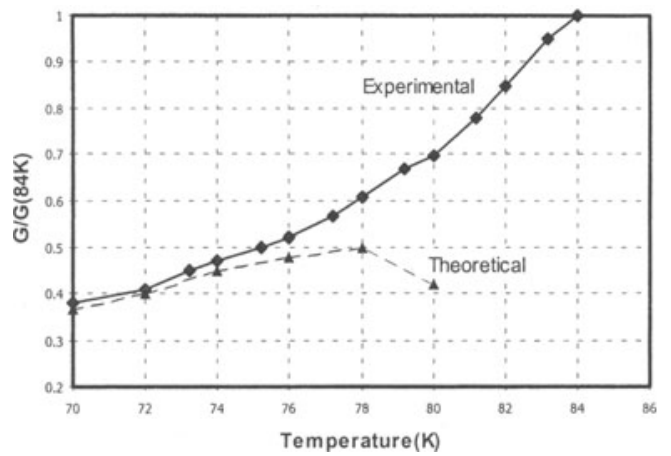

Figure 5.2. Calculated and measured normalized conductance of $200 \mathrm{~nm}$ thick $\mathrm{YBCO}$ film on $\mathrm{MgO}$ substrate versus temperature at low modulation frequencies.

materials without fluxons. It is clear that the temperatures close to the $T_{c}$ can easily lead to Abrikosov vortexes in the films. In the equilibrium, fluxons are present in the film and move due to the thermal activations. The optical photons also create additional fluxons and the flux motion (creep and flow). This factor can lead to the no bolometric component in the optical response, not considered in the above calculations. The discrepancy between theory and experiment can also be related with flux dynamics near $T_{c}$. This argument is suggested by the experimental data from [9]. As mentioned before, for the samples with sharper transition, the change in the phase of the response and the discrepancies at low frequencies are smaller. One of the possible reasons for broadening of the transition into superconducting state is the penetration of the fluxons into the samples. Another possible improvement of the model is connected with using realistic behavior of the order parameter close to $T_{c}$ and $d$-wave characteristics of the pairing in $\mathrm{YBaCuO}$ compounds [66]. On the other hand, influence of the order parameter relaxation near $T_{c}$ on the bolometer characteristic should also be taken into account for further accurate calculations near critical temperature.

\section{B. High frequency regime}

The variation of the phase of the response versus temperature at high modulation frequency (e.g. $\omega=10 \mathrm{KHz}$ ) is shown in Figure 5.3. At frequencies above the knee frequency, $\mathrm{f}_{\mathrm{L}}$, the phase of the response decreases as the temperature is lowered. To describe the heat transfer in this regime, one can imply the phonon radiation limit [62] (ballistic regime of propagation of phonons). In the high frequency regime all phonons from the superconducting film are assumed to transmit across the film-substrate boundary and absorbed in the substrate media. That is in equation (5.2) transmission probability $t_{k j}$ can be considered one. Such approximation is valid due to low Kapitza resistance of the film-substrate boundary [18]. As shown by calculations [67], the thermal conductivity in high- $T_{c}$ superconducting films has different behavior in contrast to conventional low temperature superconductors. In cuprate compounds thermal conductivity below $T_{c}$ versus temperature has a peak near the $T_{c}$ which is lowered by decreasing the film thickness [67]. The thermal conductance of the studied devices at high modulation frequencies is previously discussed to be mainly governed by thermal conductivity of the substrate material taking into account the processes in superconducting film. The thermal conductivity of the substrate can also increase as the temperature decreases 


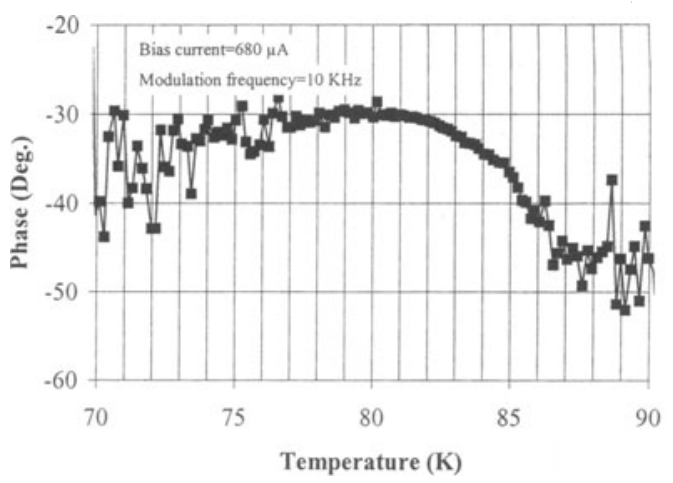

Figure 5.3. The phase of the response versus temperature at high modulation frequency of $10 \mathrm{KHz}$ and $680 \mathrm{~mA}$ dc bias current.

below the $T_{c-o n s e t}$. This is due to enhancement of the mean free path of phonons emitted from the superconducting film into the substrate. As a result the phase of the response in the temperature interval $75 \mathrm{~K}<\mathrm{T}<85 \mathrm{~K}$ decreases and at lower temperatures $\mathrm{T}<75 \mathrm{~K}$ increases. The corresponding amplitude of the response at high frequencies is shown in Figure 5.4. Response at temperatures $\mathrm{T}<75 \mathrm{~K}$ can be closer to the $\mathrm{dR} / \mathrm{dT}$ curve. The discrepancy in amplitude of response in the temperature interval $75 \mathrm{~K}<\mathrm{T}<85 \mathrm{~K}$ can also be related to the variation of the thermal conductivity in the substrate material.

In brief, at low modulation frequencies, a shift of the position of the recombination phonons in the spectrum by changing the temperature leads to decreasing of the boundary conductance of the substrate/cold-head interface for $T<T_{c}$. This with variation of the heat capacitance versus temperature leads to the agreement between the theoretical and experimental results of temperature dependence of the response of the bolometers. However, such model is not adequate at temperatures close to the critical temperature at low modulation frequencies. One reason for such behavior is related with creating fluxons and their dynamics near $\mathrm{T}_{\mathrm{c}}$.

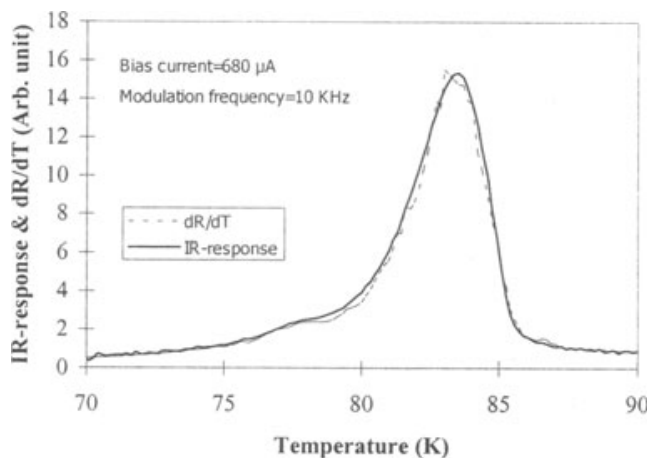

Figure 5.4. Amplitude of the response versus temperature at $10 \mathrm{KHz}$ modulation frequency and $680 \mathrm{~mA}$ dc bias current. 


\section{DC CHARACTERISTICS OF THE BOLOMETERS AND ARTIFACTS RELATED TO JOULE HEATING AND THE FILM MICROSTRUCTURE}

In most of the dc characterizations of the patterned superconducting films there can be considerable Joule heating associated with the resistance of the patterns at high bias currents. This power dissipation can cause a temperature gradient across the thermal conducting path. Hence there can be an error in the dc characterizations versus temperature caused by the above temperature gradient. This is because in most of the setups the temperature of the cold-finger (holder) or the underneath of the substrate is monitored [6]-[11], [19]-[24], [42]-[46] where the temperature of the film may be higher [11], [23]. At high bias currents this can cause a shift of $\mathrm{T}_{\mathrm{C} \text {-onset }}$ toward the lower temperatures in the measured $\mathrm{R}$ versus $\mathrm{T}$ curve as observed in many different works [21], [23]-[24], [42], [45], to be discussed later in this section. This can be either due to the temperature gradient across the film-substrate or the substrate-cold finger interfaces, depending on the film and the substrate dimensions. This effect has been a misleading parameter in the analysis of the $\mathrm{dc}$ characteristics and the $\mathrm{T}_{\mathrm{C}}$ stability of the films [19], [20], [23], [42], [46].

Also effects of the mechanical stresses on the film under different atmospheres caused by the temperature gradients associated with Joule heating, has been a degrading factor for the quality evaluations of the samples [7], [47]. These effects are found to depend strongly on the dissipation of the Joule heating powers in the samples, hence making the dc thermal conductance, $G(0)$, a determining factor for even dc characterizations.

One measure of the quality of the high- $\mathrm{T}_{\mathrm{C}}$ superconducting films has been the presence of precipitation. The effect of the microstructure and the amount of precipitation in the film on the dc characteristics of the samples have been studied and reported by many different groups. Here we also discuss present a possible effect of the precipitation on the normal resistance of the samples due to their possible contributions in the electrical conduction at relatively high bias currents. The response of the samples in this study and their potential improvement are linked to the dc effects indicated.

\section{A. Joule heating effects}

The effect of Joule heating in the superconductive films, which is equal to $I^{2} R$, depends on the heat conduction path determining the temperature variations in the sample. Since the heat is generated in the film, the film-substrate contact area is one determining factor. Also the heat conduction through the substrate into the temperature reservoir is another factor to be considered. This also takes the thermal conductivity and heat capacity of the substrate materials into consideration. For the dc characterizations under stable conditions, the consideration of the heat capacitance of neither the film nor the substrate is necessary [7]. To study the effect of Joule heating in the samples, one can divide them into two main categories, with respect to the amount of the generated heat and mechanism of the heat conduction from the film to the temperature reservoir. Then the samples can be considered as small or large area patterns compared to the dimensions of the substrate. The large area patterns are normally made of a meander lines where the small area patterns can be considered for most of the micro bridges consisted of a strait narrow path of the superconducting film.

a) Small area patterns

For microbridges with dimensions small compared to the substrate dimensions, the generated temperature gradient due to the Joule heating is mostly expected to be at the film- 
substrate interface or across a thin layer of the substrate at the vicinity of the superconducting path [23], [30]-[50]. The reasons for this are because only a small area of the substrate underneath the pattern conducts the heat, where the substrate would behave like a heat bath for the generated heat in the pattern, because of its large dimensions compared to that of the pattern. In samples with above configurations, the heat conductivity of the substrates is the determining factor in temperature gradient. For $\mathrm{R}$ versus $\mathrm{T}$ measurements of such microbridges, the temperature rise in the film can cause a thermal runaway over heating the whole film if a constant current source is used [1], [5], [24], [44]. If the measurement is monitored by a voltage controlled source then the overall heating will be limited by a local thermal runaway creating a hot spot in the bridge [24], [51]-[52]. Based on thermal stability, the hot spot would be at a point of the bridge where it can have the maximum thermal conductance, G. Hence, despite of some expectations [24] it won't happen in the middle of the bridge. Taking the thermal conduction through the film into the consideration as well, the hot spot would be located at either end of the microbridge where it has the maximum thermal conductance as reported [24] The samples studied here are not among the above category.

\section{b) Large area patterns}

For the samples with dimensions of patterned superconducting film comparable to that of the substrates, the temperature gradient is mainly across the substrate-cold finger interface [7], [16], [24]. Hence the whole pattern of the above samples is at the same temperature under any stable circumstances [7], [24]. The thermal boundary resistance at this interface (substratecold finger) is the determining factor in the overall thermal conductance of our samples [7], [24]. In such samples, there won't be a hot spot. The heat conduction is blocked mainly by the substrate-cold finger interface, giving an almost uniform heat distribution over the superconducting pattern. This is while the total thermal resistance of the samples is a few orders of magnitudes higher than the values just across the substrates [16], [24]. So, the whole superconducting film can be considered at the same temperature to a very good approximation.

At high bias currents there can be a stable heating by the complete path of the pattern creating a higher temperature in the film where the monitored temperature of the cold-finger may show lower values. This temperature rise in the film is proportional to the resistance of the film given as [7], [24];

$\Delta \mathrm{T}=\frac{\mathrm{RI}^{2}}{\mathrm{G}(0)}$.

In $\mathrm{R}$ versus $\mathrm{T}$ characterizations using a constant current source, this can cause a false sharper transition at high currents. At values close to $\mathrm{T}_{\mathrm{C} \text {-onset, }}$ as resistance of the film increases, the Joule heating increases and creates higher $\Delta \mathrm{T}$ with respect to the temperature of the cold-finger. Figure 6.1 shows this effect for one of the samples.

This false sharp transition by shifting the measured $\mathrm{T}_{\mathrm{c} \text {-onset }}$ to lower temperatures is interpreted to be an intrinsic shift by some groups [19], [23], [42], [46]. This shift increases the $\mathrm{dR} / \mathrm{dT}$ value of the film obtained from the measured $\mathrm{R}$ versus $\mathrm{T}$ curves which is measured to be different from the real $R$ versus $T$ of the film at high bias currents. One way to investigate this effect is by use of the responsivity of these samples to radiation intensities (i.e. measuring $\Delta \mathrm{R}$ due to known values of absorbed radiation) [7]. The responsivity of such patterns used as bolometers, calculated based on this unreal high $\mathrm{dR} / \mathrm{dT}$, will lead to misleading values much higher than the possible values for such configurations [22]-[23]. In granular samples with wide normal to superconducting transition, the widening of the 


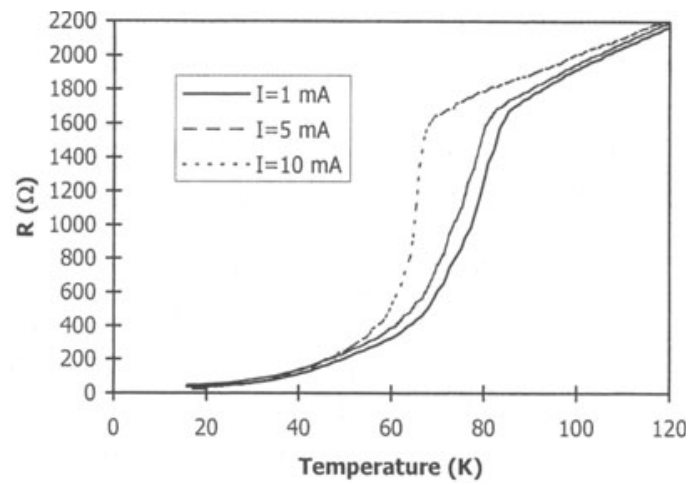

Figure 6.1. $\mathrm{R}$ versus temperature of $\mathrm{LaAlO}_{3}$ substrate sample $057-04 \mathrm{~b}$ at $1 \mathrm{~mA}, 5$ $\mathrm{mA}$, and $10 \mathrm{~mA} \mathrm{dc}$ bias currents, and $2 \mathrm{~K} / \mathrm{min}$ heating rate.

transition due to high bias currents dominates the shift of the $\mathrm{T}_{\mathrm{c}-\mathrm{onset}}$ to the lower temperatures, lowering the maximum magnitude of the $\mathrm{dR} / \mathrm{dT}$ curve showing the expected changes of the $\mathrm{dR} / \mathrm{dT}$ at higher bias currents.

\section{B. Effect of ambient atmosphere and the Joule heating}

Some samples show $\mathrm{T}_{\mathrm{C}} \mathrm{S}$ sensitive to bias currents shifting to lower temperatures, after being exposed to high currents. Such a shift would dependent on the maximum applied current determining the maximum temperature gradient in the device due to the Joule heating. This extreme change of $T_{c}$ has been mostly observed in samples with low and relatively sharp transition temperatures [24], [68]. The transition region at the $\mathrm{T}_{\mathrm{C}}$ of $\mathrm{YBa}_{2} \mathrm{Cu}_{3} \mathrm{O}_{7-\mathrm{x}}$ films on $\mathrm{LaAlO}_{3}$, and $\mathrm{SrTiO}_{3}$ substrates are typically sharper than the films on $\mathrm{MgO}$ substrates where the $T_{c}$ of the later one is reported to be more stable [7]. When these type of samples with extreme low $T_{c}$ due to high bias currents are exposed to room temperature at atmosphere pressures, the $T_{c}$ of them reaches higher values close to the initial values measured in first cycles under low bias currents. This is interpreted partly to be caused by the strain under vacuum where the strain is due to the temperature gradient in the film and the substrate. This temperature gradient is caused by Joule heating at relatively high bias currents in the normal state and in the transition region close to the $\mathrm{T}_{\mathrm{c}-\mathrm{onset}}$.

The strains under vacuum can cause release of oxygen (outgasing) in imperfect $\mathrm{YBa}_{2} \mathrm{Cu}_{3} \mathrm{O}_{7-\mathrm{x}}$ crystalline structures, decreasing the $\mathrm{T}_{\mathrm{c}}$ by decrease in the over all oxygen content. This effect can be observed when the sample is biased at a constant temperature of the cold-finger, under variable applied bias currents. This configuration is used for the measurements of the dc thermal conductance of the samples [5], [7], [24]. The result of such a measurement for sample 064-01a is shown in Figure 6.2, showing both changes due to the temperature rise in the film with respect to the cold-finger and the changes of $T_{C}$ due changes of the oxygen stoichiametry (shift of $\mathrm{T}_{\mathrm{C}}$ ) of the sample. As observed in the figure the later one that happens at bias currents over about $5 \mathrm{~mA}$ in this sample is not recovered when the temperature rise due to Joule heating is not present anymore (i.e. $I \approx 0$ ). Considering the measured $3 \mathrm{~mW} / \mathrm{K}$ for the $\mathrm{G}(0)$ of this sample the temperature rise caused by the Joule heating at a bias current of $5 \mathrm{~mA}$ is about $37 \mathrm{~K}$. Some samples with initially unstable $\mathrm{T}_{\mathrm{C}}$ are reported 


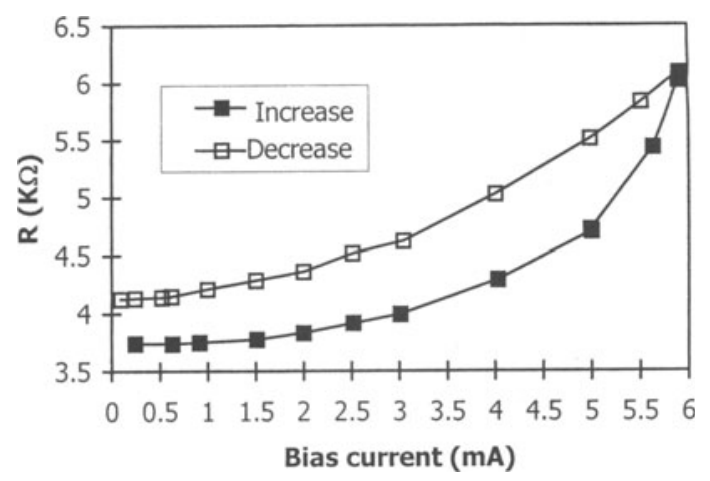

Figure 6.2. R vs. $\mathrm{I}_{\text {bias }}$ of sample 064-01a at a bias temperature of $97 \mathrm{~K}$, measured in two directions of increasing (filled squares), and decreasing (empty squares) $\mathrm{I}_{\text {bias }}$.

to reveal a stable and higher $T_{C}$ after being exposed to high mechanical stresses under ambient atmosphere [25]. This is interpreted to be due to the release of undesired strains existing in the film from the growth of the superconducting crystalline film on the substrate.

\section{Effect of microstructure and bias current}

There are also repeatable and stable changes in the magnitude of the normal resistivity of some samples in the $\mathrm{R}$ versus $\mathrm{T}$ measurements, which happens by changing the bias current as shown in Figure 6.3. This change of the magnitude of resistance versus bias current is apart from that due to the shift of $\mathrm{T}_{\mathrm{C}}$ and the changes discussed before. Two major effects are the cause for this type of dependence of the normal resistance, $\mathrm{R}$, on the bias current that is observed even for the samples with stable $T_{c}$. Some of these changes are due to the instrumentation in the $R$ versus $T$ characterization systems using constant current bias. This is partly caused by non-ideality of the current sources specifically in the systems where the current sources are made of a resistor and a battery. This configuration is essential for ultra low noise measurements of the signal, which is discussed in next section.

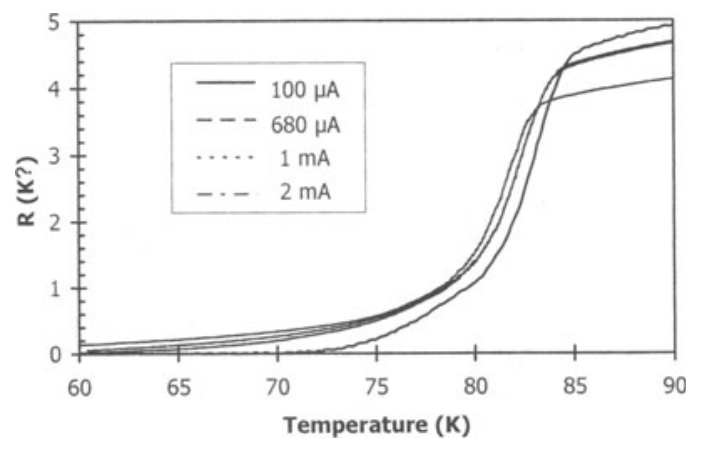

Figure 6.3. R versus temperature of sample $064-02 \mathrm{~b}$ at $100 \mu \mathrm{A}, 680 \mu \mathrm{A}, 1 \mathrm{~mA}$, and $2 \mathrm{~mA}$ dc bias currents with $2 \mathrm{~K} / \mathrm{min}$ heating rate. 


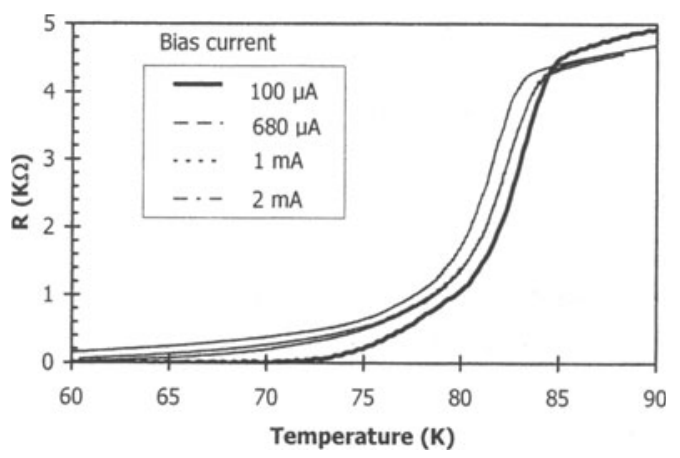

Figure 6.4. $\mathrm{R}$ vs. temperature curves of sample $064-02 \mathrm{~b}$ (in Figure 5) at $100 \mu \mathrm{A}$ to 2 $\mathrm{mA} \mathrm{I}_{\text {bias }}$, corrected for errors caused by the impedances of the source and the device.

Knowing the output impedance of the current source and the relative changes of $\mathrm{R}$ versus $\mathrm{T}$ at the transition, this error can be corrected. The $\mathrm{R}$ versus $\mathrm{T}$ of sample $064-02 \mathrm{~b}$ in Figure 6.3 corrected for this effect is shown in Figure 6.4. As shown in Figure 6.4 there still exist a dependence of $\mathrm{R}$ on bias current showing a nonlinear decrease of the normal resistance when the current is increased. This decrease of the $R$ at higher bias currents (at the same $T$ ) is reverse of the expected change due to shift of $T_{C}$ caused by Joule heating.

As shown in Figure 6.4, there is a change in the normal state $\mathrm{R}$ much deviating from the predicted values due to the shift in the $R$ versus $T$ curve. This lowering of the normal state resistance at higher bias currents is observed in highly granular samples. There can be at least two possible mechanisms for this phenomenon. One can to be due to high voltage drops across the precipitation that may have comparable resistance with the shunt superconducting paths in the normal state. Then the nonlinear dependency is due to possible minimum threshold voltage values required at the boundaries for passing the current through the potential barriers at the boundary (some kind of metal-semiconductor tunneling mechanism and or metal-semiconductor junction). Another can be due to a possible tunneling mechanism at the week links mainly created during the patterning process. Due to high precipitation in the film there can be some undesired etching around the large particles of the precipitation. The further increase of current $(\mathrm{I}>100 \mu \mathrm{A})$ in Figure 6.4 which is not showing further considerable changes in the normal state resistance, is consistent with the above analysis. After the above considerations there still is a shift in the $\mathrm{T}_{\mathrm{C} \text {-onset }}$ to lower temperature at higher bias currents, which, as discussed before, is due to the Joule, heating in the film and the limited G.

As observed in Figure 6.4, there is also an excessive decrease in $T_{\text {c-zero }}$ at high bias currents, which increases the width of the transition region. This excessive widening of the transition which occurs in highly granular samples can dominate the sharpening of the

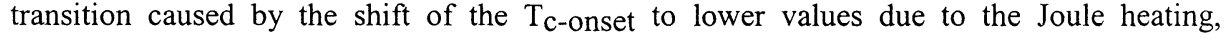
increasing the over all width of the measured transition. The $\mathrm{dR} / \mathrm{dT}$ of the $\mathrm{R}$ versus $\mathrm{T}$ curves in Figure 6.4 are shown in Figure 6.5. As is observed in the figure, there is a shift of $\mathrm{T}_{\mathrm{C} \text {-onset }}$ of about 2 degrees at $2 \mathrm{~mA}$ bias current toward the lower temperature. The sharpening of the transition region due to the shift of $T_{c-o n s e t}$ to lower values in the measured $R$ versus $T$ curves does not have observable effect on the real magnitude of the $\mathrm{dR} / \mathrm{dT}$ at any point, which is investigated using the measured bolometric response of the samples. Though the measured 


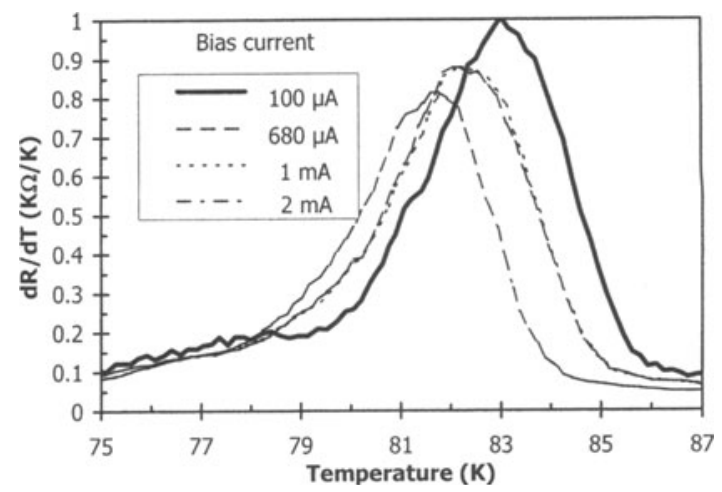

Figure 6.5. $\mathrm{dR} / \mathrm{dT}$ versus temperature of sample $064-02 \mathrm{~b}$ at $100 \mathrm{~mA}$ to $2 \mathrm{~mA} \mathrm{dc}$ bias currents, derived using the $R$ versus $T$ curves with a $2 \mathrm{~K} / \mathrm{min}$ heating rate.

shift at high bias currents due to the granularity of the film lowering the $T_{c-z e r o}$, is real and intrinsic lowering the real peak magnitude of the $\mathrm{dR} / \mathrm{dT}$ of the samples that was also investigated using the bolometric response of the samples, discussed in following section.

\section{Determining dc characteristics using current normalized response}

For the measurement of the bolometric response versus temperature of the cold-finger at different bias currents (i.e. the temperature of the film can be different due to Joule heating),

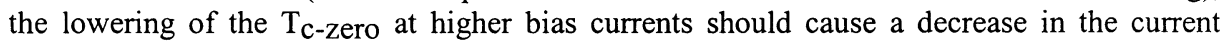
normalized response of the bolometers [24]. Where the shift due to the Joule heating only shifts the measured temperature of the onset of the transition and the peak temperature of the response (and also the $\mathrm{dR} / \mathrm{dT}$ of the film) with respect to the temperature of the cold-finger (i.e. the monitored temperature). This temperature shift due to the Joule heating is toward lower temperatures and is higher at higher bias currents and higher temperatures. This shift can be found and corrected for, using equation (6.1). The current normalized response of sample No. 064-02b with the above characteristics is shown in Figure 6.6. This shows the real

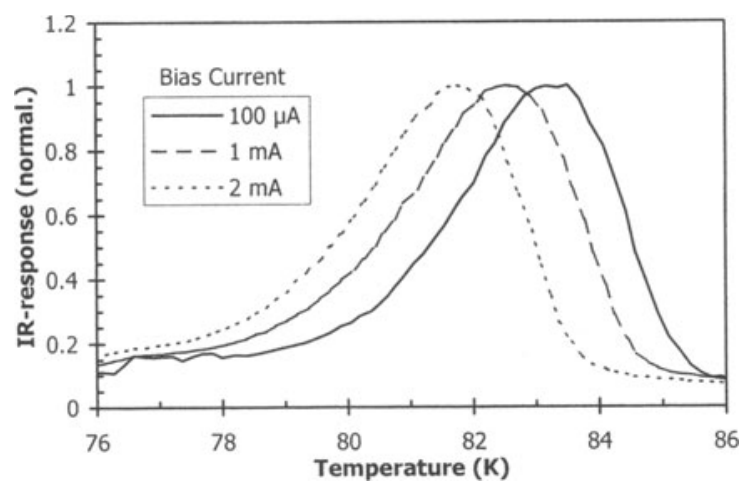

Figure 6.6. Normalized response of sample $064-02 \mathrm{~b}$, at $100 \mu \mathrm{A}, 1 \mathrm{~mA}$, and $2 \mathrm{~mA}$, and $10 \mathrm{KHz}$ modulation frequency and $2 \mathrm{~K} / \mathrm{min}$ heating rate. 


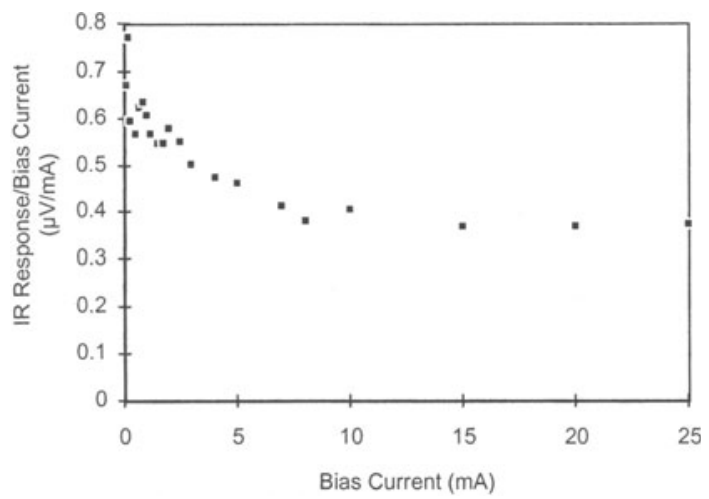

Figure 6.7. Current-normalized responsivity of a highly granular-film bolometer vs. dc bias currents at $50 \mathrm{~Hz}$ modulation frequency $=50 \mathrm{~Hz}$.

$\mathrm{dR} / \mathrm{dT}$ curve at different bias currents of Figure 6.4, since for the bolometric response we have:

$r_{v}=\eta I \frac{d R}{d T}$

As shown in Figure 6.6, the peak of the response is shifted to lower temperatures due to Joule

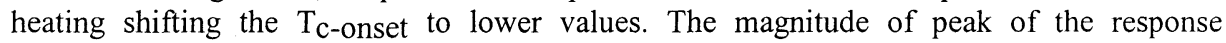
measured as $386 \mathrm{nV}, 3.28 \mu \mathrm{V}$, and $5.87 \mu \mathrm{V}$ for $100 \mu \mathrm{A}, 1 \mathrm{~mA}$, and $2 \mathrm{~mA}$ bias currents respectively, shows a slight lowering due to the shift of $\mathrm{T}_{\mathrm{c}-\mathrm{z} \text { ero }}$ to lower temperatures following the changes in the maximum $\mathrm{dR} / \mathrm{dT}$ curves of Figure 6.5 . These changes in the magnitude of the response due to real changes of $\mathrm{dR} / \mathrm{dT}$ and the peak temperature caused by the temperature rise of the film with respect to the substrate or the cold finger is interpreted as an effect of intrinsic response of the high- $\mathrm{T}_{\mathrm{C}}$ superconducting bolometers by some groups [22]-[23], [46]. Based upon the above analysis, such an interpretation is not warranted. The misinterpretation of the shift of $\mathrm{T}_{\mathrm{c}-\mathrm{onset}}$ and the dc characteristic of the samples can also mislead the responsivity calculations by two orders of magnitude higher, as obtained by some groups [23]. It can also cause the equilibrium response of such a devices to be mistaken by no equilibrium responses [22].

The dc characteristics of the thick films are more sensitive to both the environments and the bias currents. This is mainly to high granularity of this type of films since they are fabricated by post-annealed screen-printed pastes made of powders on polycrystalline substrates. [29], [4] As shown in Figure 6.7, the current-normalized responsivity of such detectors drops with increase of the bias current up to current densities even near $800 \mathrm{~A} / \mathrm{cm}^{2}$. This effect is attributed to the granular structure of the films. The superconducting material at the grain boundaries can be partially driven to the normal state, resulting in a decrease in the sensitivity of the film [25].

\section{NOISE CHARACTERISTICS AND SPECTRAL DETECTIVITY OF YBCO SUPERCONDUCTOR BOLOMETERS}

The ultimate sensitivity of a superconductor infrared bolometer is determined by its noise characteristics, which is dependent on the operating temperature, bias current, and modulation 
frequency [7]. A parameter such as responsivity is important in that one wishes to detect the smallest possible signals, limited by the noise from the detector called intrinsic noise. One criterion for the responsivity is to be large enough to increase the signal and the intrinsic noise from the detector, above that from the system, such as the noise from preamplifier, circuit elements, etc. Then the parameter noise equivalent power (NEP) together with the responsivity determine the directivity, $\mathrm{D}^{*}$ as given in equation (7.1). The NEP is defined as the input signal power that would give the noise observed in the output. Ultimate performance in a detection system is achieved when the noise generated by the background photon flux, incident on the IR-detector is larger than any thermally generated noise within the detector [1], [9], [11].

The detectivity of bolometers is basically a function of the bias current, the absorbing area (and its absorption coefficient), and the bias temperature through the slope of the resistance versus temperature curve, $\mathrm{dR} / \mathrm{dT}$. The detectivity versus temperature can be written as a function of the responsivity and the noise as:

$D^{*}=\frac{\sqrt{A \cdot \Delta f} \cdot r_{v}}{V_{n}}$

where $A$ is the radiation absorbing area of the detector, $r_{v}$ is the responsivity, $V_{n}$ is the voltage noise, and $\Delta \mathrm{f}$ is the frequency range used to obtain the noise measurement.

\section{A. Noise in high $\mathbf{T}_{\mathbf{c}}$ superconducting materials}

There has been considerable work on the noise characteristics of the superconducting materials, most of which has been focused on the low frequency or $1 / \mathrm{f}$ flicker noise. The study of noise in the high $\mathrm{T}_{c}$ materials may give important information about the physics of the transition [69]-[70]. This includes the onset of the several stages of the transition, the intergrain critical currents, the effect of a magnetic field, the role of inhomogeneities, and other kinds of disorder, and the dynamics of the vortex motion in the material [71]. Noise measurements due to conductivity fluctuations (or resistance fluctuations) reported by different groups [42], [49] have given the following main results; (a) no noise is found in the superconducting state well below $\mathrm{T}_{\mathrm{C}}$, (b) in the normal state near $\mathrm{T}_{\mathrm{c}}$, the noise is large compared to metal-insulator composites, and (c) the noise was reported to increase in the transition region [71].

The effect of a defect can be modeled as a two-level fluctuator, (TLF), moving between two energy states separated by an energy barrier [72]. Then the system undergoes transitions between the two states generating a random telegraph signal (RTS). Among the various mechanisms that can be responsible for such a signal in a current biased high $T_{c}$ film, are charge trapping and flux hopping mechanisms, which can have different origins. The noise voltage generated by two-level fluctuators is frequently observed at temperatures right below $T_{c}$, and seems to be one of the universal features of these materials [73]-[74].

An anomalous increase of low frequency, 1/f, noise for epitaxial, and granular high temperature superconductors has been reported for temperatures close to the superconducting transition temperature [75]-[79]. There is general agreement on the observation of excess noise in granular, or a and c axes mixed orientation YBCO superconducting films, compared to high quality epitaxial c-axis oriented films [1], [45], [76], [77]. Proposed interpretations of the above phenomenon have been based on two ideas, a percolating network made of superconductor and normal elements [80]-[82], and the random motion of magnetic flux tubes [81]-[85]. 
Above $T_{c}$, the Cooper pairs have a finite lifetime and the conductivity is the sum of the conductivity of the normal electrons and the conductivity of Cooper pairs. Hence the conductivity fluctuations in this region can be interpreted to be due to the fluctuations in mobility and/or density of the carriers. Following the above discussion, in the lower part of the transition, another type of conductivity fluctuation is reported to be dominant in comparison to the fluctuations in number or type of carriers, and is interpreted as fluctuations of the volume fraction of the superconducting phase [86].

One of the major sources of noise in these material is the motion of flux tubes or current vortices, which can be generated by an external magnetic field, by the self field produced by current in the material, or spontaneously as pairs of opposite oriented flux tubes. The noise study in the low field, or isolated vortex regime, in high $T_{c}$ materials has shown the importance of vortex motion in low frequency noise, which is proposed to be due to thermally activated two-level or diffusive vortex motions [87]-[88].

Other mechanisms such as phase slip, a strong Lorentz-force due to an applied current, and localized weak pinning centers, have also been reported to be strongly related to the above noise characteristics [89]-[90]. It can be argued that the corresponding voltage noise at the transition temperature could be generated by the rearrangement of vortices collectively pinned by the film microstructure, which has been shown to be affected, and partly controlled by the external field [91].

In this part, results from the measurements of the noise with different frequency, temperature, and bias current dependences are discussed. Noise measurements versus temperature and bias currents are studied, and two major possible governing mechanisms for the noise behavior of the samples are discussed. For a $\mathrm{SrTiO}_{3}$ substrate sample both the spectral response and noise is given and from these the spectral detectivity of the sample is obtained. From the above results and the frequency response of the sample one can project a maximum detectivity for an optimally designed bolometer [7]. In addition, the noise seen in all the regions can serve as a sensitive characterization tool for superconducting thin films [11].

\section{B. Noise characteristics of the detectors}

Simultaneous measurement of the IR-response and the resistance versus temperature, show that the bolometric response closely follows the $\mathrm{dR} / \mathrm{dT}$ curve of the samples. The results for sample 064-02b are given in Figure 7.1. The response in this range of bias currents,

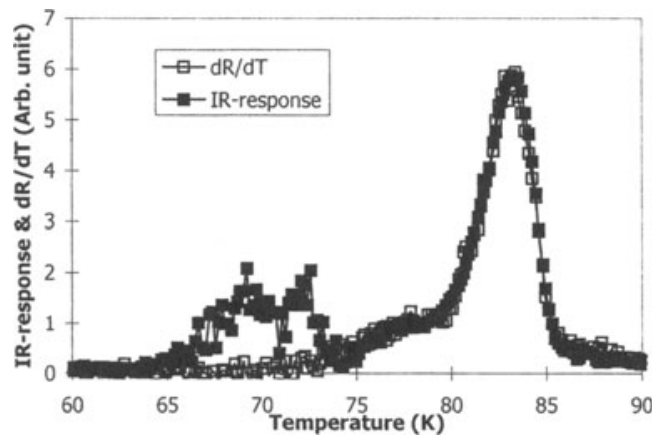

Figure 7.1. IR-response and $\mathrm{dR} / \mathrm{dT}$ vs. temperature of sample $064-02 \mathrm{~b}$, at $100 \mu \mathrm{A}$ bias currents, and $10 \mathrm{KHz}$ modulation frequency. 


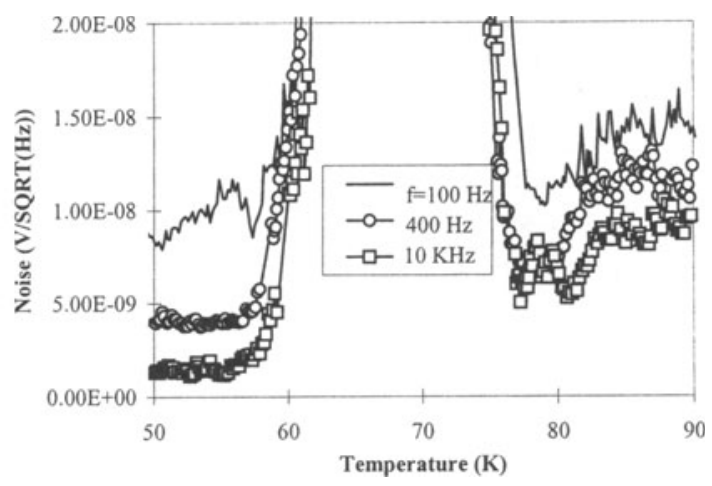

Figure 7.2. Noise voltage vs. temperature of sample $064-02 \mathrm{~b}$ at $100 \mu \mathrm{A}$ bias current.

frequency, and radiation intensity is expected to follow the $\mathrm{dR} / \mathrm{dT}$ curve [24]. As observed in Figure 7.1, a noise voltage signal, which is comparable to the bolometric response signal, occurs at temperatures close to and below $\mathrm{T}_{\mathrm{c} \text {-zero }}$, and for low bias currents is larger than the bolometric response. This signal exists even in the absence of the optical radiation, and its magnitude is also significantly affected by the intensity of the radiation. Measurements of the noise voltage versus temperature of the samples, in the four probe configuration, reveals that this voltage signal is an excess noise voltage in the material that occurs at and below $\mathrm{T}_{\text {c-zero. }}$.

The results for above noise at different frequencies and temperatures, is given in Figure 7.2. From the noise characteristics versus temperature shown in the figures, the noise in the material can be divided into two major kinds. One is the excess noise at and below $\mathrm{T}_{\mathrm{c}-\mathrm{zero}}$, and the other is the noise that follows the resistance versus temperature transition at and above

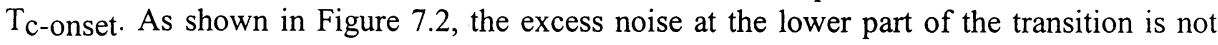
significantly affected by the frequency and has the characteristics of a white noise. The magnitude of this noise is a few orders of magnitudes higher than the calculated Johnson noise, and phonon noise [7]. The Johnson noise is calculated for $1 \mathrm{~Hz}$ bandwidth from

$\mathrm{V}_{\text {John }}=\sqrt{4 \mathrm{~K}_{\mathrm{B}} \mathrm{TR}}$

Where, $\mathrm{K}_{\mathrm{B}}$ is the Boltsman constant $\left(1.38 \times 10^{-23}\right), \mathrm{T}$ is the temperature, and $\mathrm{R}$ is the resistance of the sample at the temperature indicated. The resistance and thermal conductance of the samples are given in VI_Table 2.1. The phonon noise was calculated from:

$\mathrm{V}_{\text {phonon }}=\mathrm{r}_{\mathrm{v}} \times \sqrt{4 \mathrm{GK}_{\mathrm{B}} \mathrm{T}^{2}}$

where, $\mathrm{G}$ is the thermal conductance of the samples.

The dependence of this excess noise on the bias currents at $10 \mathrm{kHz}$, is given in Figure 7.3. As shown in the Figure 7.3, the width of the temperature region of the noise increases with an increase in the bias current and goes to lower temperatures at higher bias currents. This is can be due to the weak links and the corresponding SNS junctions. The broadening of the noise with an increase in the current can be interpreted as due to the effect of the bias current on the weak links in the film. In a granular film, or a film with weak links, additional regions at the grain boundaries or the weak links, can be driven into the normal state at lower temperatures by the increase of the current through the film. Based on the properties of the superconducting material, and the relations between $\mathrm{J}_{\mathrm{C}}, \mathrm{T}_{\mathrm{C}}$, and $\mathrm{H}_{\mathrm{C}}$, the broadening of the noise versus temperature, or in other words the lowering of the onset temperature of the noise, should also 


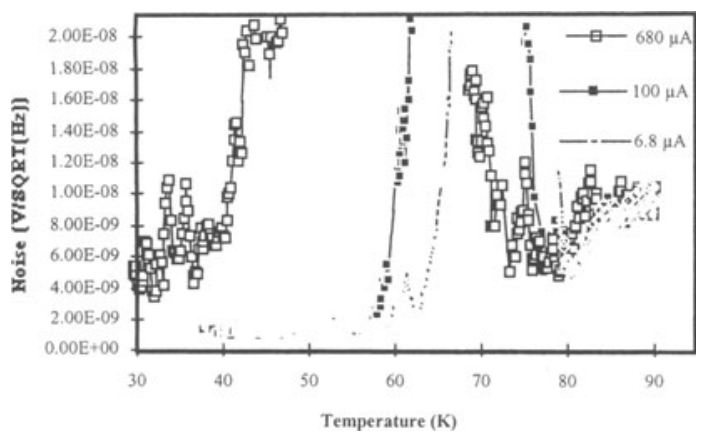

Figure 7.3. Noise voltage vs. temperature of sample $064-02 \mathrm{~b}$ at $6.8 \mu \mathrm{A}$ to $680 \mu \mathrm{A}$ and $10 \mathrm{KHz}$ modulation frequency.

occur by applying a magnetic field. The temperature onset of the noise can be considered as the temperature at which the first normal links appear in the path of the current as the temperature is increased from low temperatures. The effect of the magnetic field on this type of noise has been studied by other groups for high $\mathrm{T}_{\mathrm{c}}$ superconducting films at low frequencies [91].

The onset of the excess noise for sample No. 064-02b at different bias currents is obtained from the noise versus temperature of the sample and given in Figure 7.4 The values of $\mathrm{T}_{\mathrm{C} \text {-zero }}$ of the sample obtained from the $\mathrm{R}$ versus $\mathrm{T}$ curves, are also plotted for different bias currents. Based on this assumption the onset temperature of the noise in the above sample versus bias current should have a behavior similar to the critical current density, $\mathrm{J}_{\mathrm{c}}$, versus temperature, and Figure 7.4 shows such a correlation. Based on the above, this noise should exist even at zero bias currents, and not to be changed considerably by currents lower than some value, where the normal regions are mainly determined by the temperature. This is observed in the noise measurements versus temperature at the very low bias current close to $6.8 \mu \mathrm{A}$, which is the lowest bias current at which the reported measurement is done.

One explanation for the existence of the excess noise at very low bias currents or zero bias

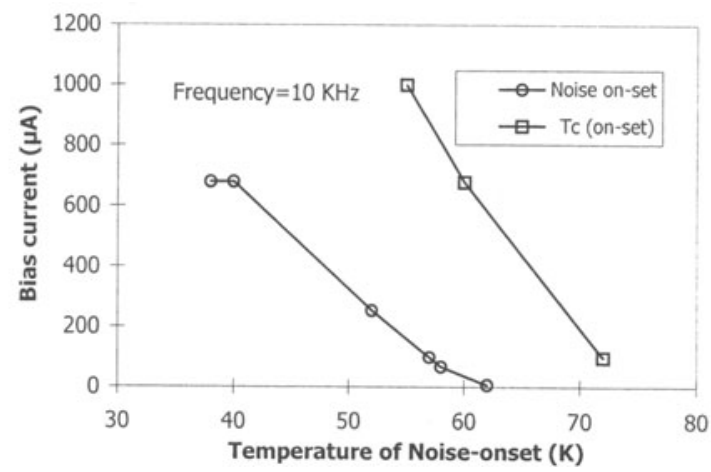

Figure 7.4. The $T_{c-o n s e t}$ and on-set temperature of the excess noise vs. bias current of sample 64-02b. 
current is as follows. Any two neighboring superconducting grains, with a normal region inbetween or a gap, can be considered as a Josephson junction (SNS junction). Then there can be a supercurrent associated with the junction as a result of the tunneling mechanism between the two superconducting grains. A granular film or material can be considered as a many junction media. Near $\mathrm{T}_{\mathrm{C}}$ these junctions can be considered in series and parallel combinations, which may not be shunted by any complete superconducting path. In such a configuration, there can be voltage fluctuations due to the fluctuating super current, even with zero bias current through the material. The onset of this noise can also be shifted toward lower temperatures by applying a bias current through the material, since only the normal regions between the grains that have high Josephson currents will carry super currents. There have also been reports on the effect of the patterning process, and the dimensions of the patterns of a design on the noise performance of the materials, which is in agreement with the above analysis. [1], [45], [91].

The effect of the bias current on the noise in the normal region above $\mathrm{T}_{\text {c-onset }}$ of the material has also been extensively studied. This noise is reported to be higher than the Johnson noise, and is current dependent at low frequencies as reported by different groups [1], [11], [45], [91]. The dependence of the noise on the bias current suggests that the noise can be due to the resistance fluctuations in the material. This noise is linearly proportional to the bias current. This suggests that the noise in the normal state is caused by resistance or conductivity fluctuation in the film and also suggests that the resistance (or conductivity) fluctuation is not dependent on the bias current. As discussed previously, above the transition temperature, the Cooper pairs have a finite lifetime, and the conductivity is the sum of the conductivity of the normal electrons and the conductivity of Cooper pairs, which can fluctuate randomly. Hence, the conductivity fluctuations in this region can be interpreted to be due to the fluctuations in the density of the different type of the carriers [92].

There has been another noise characteristic, observed at temperatures right below $T_{c-z e r o}$, in sample No. 064-03b, which is less granular than sample 064-02b [24]. This kind of noise peaks right below the onset temperature of the excess noise. Figure 7.5 shows the noise versus temperature of this sample. As observed in the figure the sample has excess noise the same as sample 064-02b, which is associated with the long tail of the $R$ versus $T$ curve due to the granularity of the sample. As shown in Figure 7.5 the noise-peak below $\mathrm{T}_{\mathrm{c}-z e r o}$, can increase with an increase in the bias current, and approaches zero for zero bias current. For very large

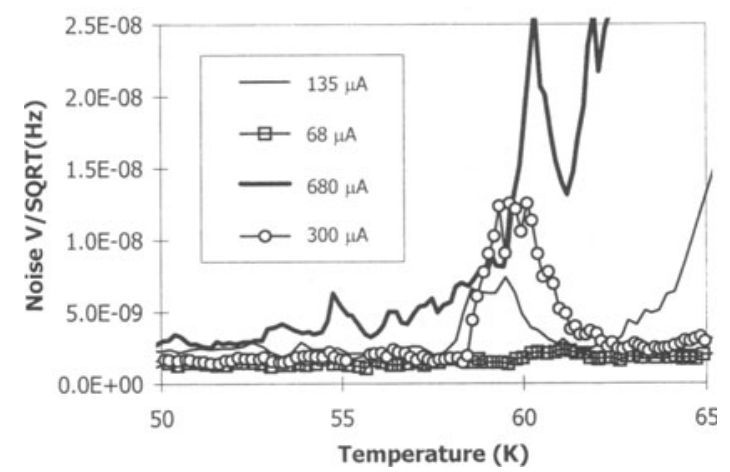

Figure 7.5. Noise voltage vs. temperature of sample $064-03 \mathrm{~b}$ at $10 \mathrm{KHz}$. 
bias currents the excess noise extends over the whole region creating other small peaks, at even lower temperatures. In the temperature region below $\mathrm{T}_{\text {c-zero, }}$, and low bias currents, a continuous superconducting path is expected to exist between the current and voltage contacts of the superconductor film in the four-probe configuration. Consideration of this, and the strong dependence of the noise on the bias current, suggests vortex motion as the source for noise in this region, which can be due to thermally activated two-level or diffusive vortex motion [87]-[88] that may also be enhanced by a strong Lorentz-force due to an applied current [89]-[90].

The noise versus temperature, for sample 064-01a, is shown in Figure 7.6. This sample has a relatively sharp transition and is less granular compared to the $\mathrm{MgO}$ substrate samples. Noise in this sample at high frequencies $(10 \mathrm{kHz}$ and $14 \mathrm{kHz})$ has shown a very small peak at $\mathrm{T}_{\mathrm{c} \text {-zero, }}$ and follows the $\mathrm{R}$ versus $\mathrm{T}$ curve in the upper part of the transition toward the normal state. The noise behavior in the normal state of this sample is the same as observed in $\mathrm{MgO}$ substrate samples. The magnitude of the peak in the noise of sample 064-01a at $T_{\text {c-zero }}$ is very small, a few nanovolts, and its maximum for the maximum used bias current of $680 \mu \mathrm{A}$, is less than 10 nanovolts $-\mathrm{Hz}^{-0.5}$. The noise in this sample is strongly dependent on the frequency, and increases rapidly for the frequencies below about $400 \mathrm{~Hz}$, as shown in Figure 7.6. This type of noise is not observed in $\mathrm{MgO}$ substrate samples. As can be observed in the Figure 7.6 the peak of the noise increases as the frequency decreases, and it is also found to be current dependent. The frequency and current dependence of the above noise, suggests that conductance fluctuations are the source of the noise in the transition region of this sample. This type of fluctuation in the lower part of the transition is interpreted to be dominated by the conductivity fluctuations, due to the variations in the volume fraction of the superconducting phase, in the current path through the material [86].

So four major types of noise can be identified according to where they occur in temperature relative to the $\mathrm{T}_{\mathrm{c} \text {-onset }}$ and the zero resistance temperature, $\mathrm{T}_{\mathrm{c} \text {-zero, }}$, and their dependence on the frequency and bias current. They are also associated to with the granularity of the superconducting films, which is partly related to the substrate material used. From the above discussion a specific cause for each type of noise is associated and the results can be

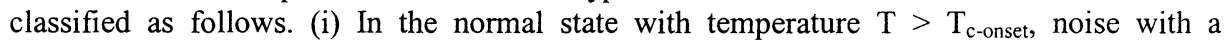
magnitude that is consistent with thermal (Johnson) noise exits, and depends linearly on bias

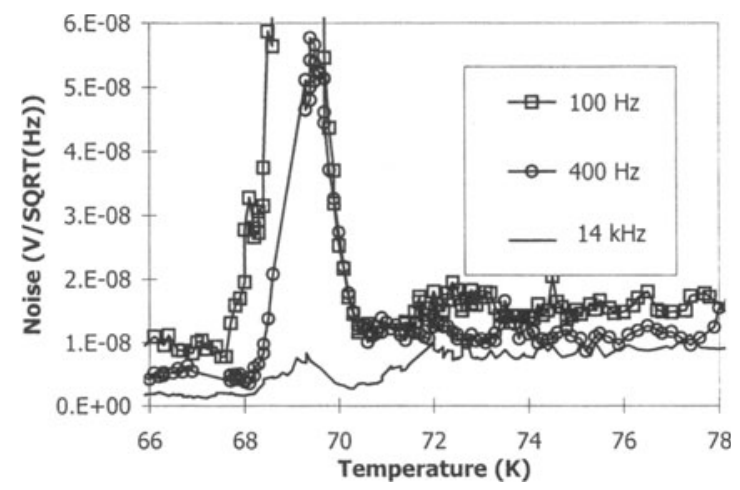

Figure 7.6. Noise voltage vs. temperature of sample $064-01 \mathrm{a}$ at $100 \mu \mathrm{A}$ bias current. 
current above a threshold value at low frequencies. The suggested noise source is conductivity fluctuations due to the Cooper pairs. (ii) A second type of noise exists above $\mathrm{T}_{\text {c-zero }}$ in granular films. With increasing the bias current, its magnitude increases, and it shifts to lower temperatures; however the noise magnitude becomes constant as the current goes to zero while is weakly dependent on the frequency for above $400 \mathrm{~Hz}$. This is suggested to be caused by voltage fluctuations in the superconductor-normal-superconductor junctions at grain boundary. (iii) One type of noise occurs below $\mathrm{T}_{\mathrm{c} \text {-zero }}$ with peaks at various temperatures. With increasing the bias current the peaked noise spreads to lower temperatures, but the noise goes to zero as the current goes to zero. This can be caused by the magnetic flux tube motions. (iv) A noise signal occurs between $\mathrm{T}_{\mathrm{c} \text {-zero }}$ and $\mathrm{T}_{\mathrm{c} \text {-onset }}$ and is present in al bolometers. Its suggested cause is fluctuations in the volume fraction of the superconducting phase along the current path. This type of noise is the limiting factor in the detectivity of the edge transition bolometers as it occurs in the range of interest for the bias temperature of the devices.

\section{CONTROL OF RESPONSIVITY AND DETECTIVITY THROUGH SUBSTRATE PROPERTIES}

The thermal resistances at the substrate-cold finger and the superconductor-substrate interfaces need to be used in the design considerations to improve the detectivity, $\mathrm{D}^{*}$. For an optimal design, both electrical and thermal properties of the detectors should be considered. The optimal design parameters are also strongly dependent on the application and the operating circumstances, mainly the modulation frequency. In this section we discuss the analysis for the response and $\mathrm{D}^{*}$ of samples with large area patterns on crystalline $\mathrm{MgO}$, $\mathrm{LaAlO}_{3}$, and $\mathrm{SrTiO}_{3}$, under dc bias current in the four probe configuration.

\section{A. Effects of substrate properties and biasing on the responsivity and detectivity limits}

The responsivity of a bolometer is defined as the ratio of the voltage response (in current biased detectors) to the radiation power received by the detector [7]-[24]. The equilibrium responsivity of thin film superconductive edge transition bolometers is mainly a function of the substrate properties, since the mass of the substrate material is usually much larger than the other masses in this type of the detectors. The thermal and structural characteristics of the substrate also affect the electrical properties of the superconducting film, which is considered later in this section as well [95]-[98]. The electrical biasing is also a major determining factor in the response, and it needs to be considered before further discussion of the effects of substrate properties on the response.

a) Responsivity and effects of biasing configurations

Self or Joule heating is a major factor in the bolometric response, since the bolometer operates within the resistive transition region of the superconductor near the maximum in $\mathrm{dR} / \mathrm{dT}$. The ac component is caused by the change in the operating point resistance due to the input radiation power, and produces positive feedback for current biased (dc) detectors in the four-probe measurement configuration. Taking the above effect into account in the equation for the responsivity of bolometers [7], the overall frequency dependent responsivity, given in equation (2.6) can also be obtained directly from [9];

$r_{v}=\frac{h_{b}}{G_{t}+j 2 \pi f C_{t}-I_{b}^{2}(d R / d T)} \frac{d R}{d T}$,

Where $\mathrm{Ib}$ is the dc bias current, $\eta$, is the fraction of the incident power absorbed by the bolometer (the absorption coefficient), $\mathrm{dR} / \mathrm{dT}$ is the slope of the resistance versus temperature 
( $R$ vs. T) curve at the bias point $\mathrm{G}_{t}$ and $\mathrm{C}_{t}$ are the frequency dependent total thermal conductance and heat capacity of the bolometer, respectively, which are mainly governed by the substrate properties in most of the cases [7]. As shown in equation (8.1), there is a stability criterion in the voltage response, which has the same form as obtained for dc biasing at zero frequency [7].

For dc voltage biased detectors (constant voltage across the samples), the response is in the form of current variations, and the Joule heating in the film produces negative feedback [12]. This decreases the responsivity, changing the sign of the Joule heating term $\left(\mathrm{I}_{b}{ }^{2}(\mathrm{dR} / \mathrm{dT})\right)$ in equation (8.1). This negative feedback can improve the dynamic range of voltage-biased detectors by stabilizing the bias temperature at high radiation intensities. This is when the sample is biased such that the Joule heating is comparable to the total absorbed radiation power.

\section{b) Effects of substrate dependent film properties on the detectivity, $D^{*}$}

The detectivity, $\mathrm{D}^{*}$, of the bolometers is a function of responsivity, the absorbing area (and its absorption coefficient), and the intrinsic noise in the device. The $\mathrm{D}^{*}$ of a edge transition superconductive bolometer versus temperature is a function of the voltage responsivity and the noise as defined in equation (7.1). Using equation (8.1), $\mathrm{D}^{*}$ might be increased by an increase in the responsivity by using higher dc bias currents. This depends on the dependence of the ultimate responsivity and the noise voltage on the bias current, as discussed in the following.

When ac Joule heating is negligible, as is the case for the currents in our samples, the responsivity increases linearly with $\mathrm{Ib}_{b}$ as given in (8.1). Hence $\mathrm{D}^{*}$ can be increased by increasing $\mathrm{Ib}$ if the signal to noise ratio also increases. This requires the noise increases with $\mathrm{Ib}$ at a rate less than that of the responsivity. Noise in $\mathrm{YBa}_{2} \mathrm{Cu}_{3} \mathrm{O}_{7-\mathrm{x}}$ samples has a dependence on the bias current and the modulation frequency, and also is strongly temperature dependent as discussed in the preceding section. The $\mathrm{T}_{\mathrm{c} \text {-onset }}$ is considered here to be the point above which the resistance starts to show linear behavior versus temperature, and $\mathrm{T}_{\text {c-zero }}$ the point

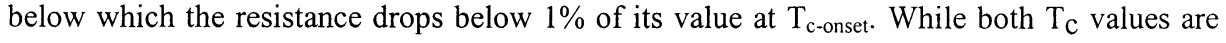
dependent on the bias current, $\mathrm{T}_{\text {c-zero }}$ of the studied is decreased versus bias current depending on the granularity of the films [7]. The measured voltage noise versus temperature at different Ib values in the region of interest for one of the samples (064-02b) made of 120-130 nm thick patterned granular $\mathrm{YBa}_{2} \mathrm{Cu}_{3} \mathrm{O}_{7-\mathrm{x}}$ film on crystalline $\mathrm{MgO}$ substrate is shown in Figure 8.1.

The noise from about the middle of the transition to $\mathrm{T}_{\mathrm{c} \text {-onset }}$ is reported to be dependent on the bias current, $\mathrm{I}_{\mathrm{b}}$, scaling as [9];

$\mathrm{V}_{\mathrm{n}}=\mathrm{C}_{1}+\mathrm{C}_{2} \mathrm{I}_{\mathrm{b}}$

Where $C_{1}$ and $C_{2}$ are constants and differ from one sample to another, with the $C_{1}>C_{2} I_{b}$ relation just below $T_{\text {c-onset }}[11]$. The noise in the $\mathrm{LaAlO}_{3}$ samples is reported to be typically higher in this region. The high level of noise in films on $\mathrm{LaAlO}_{3}$ substrates can be due to the high granularity (twined grains) typical of this type of the substrate material. Considering the current dependence of the noise, substrate samples having sharper transitions (higher $\mathrm{dR} / \mathrm{dT}$ ), which lead to lower $I_{b}$ values for the maximum responsivity, are more favorable in this respect. The highest $\mathrm{dR} / \mathrm{dT}$ is also found to be at the lower end of the above noise temperature region [11]. Hence, since the responsivity is proportional to $I_{b}$, the $D^{*}$ of samples increases by increasing $I_{b}$ if the sample is biased in the above temperature region. This increase is limited 


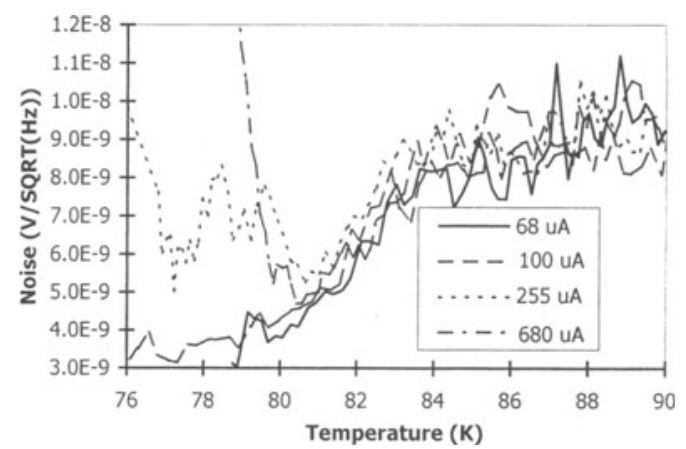

Figure 8.1. Noise vs. Temperature of sample $\mathrm{MgO}$ substrate $064-02 \mathrm{~b}$ at 10

$\mathrm{KHz}$ for $68,100,255$, and $680 \mu \mathrm{A}$ bias currents.

by the thermal runaway due to dc Joule heating in the device, and the limits to the responsivity which are discussed later in this section.

c) Effects of substrate properties and bias current on responsivity limits

Due to the limited thermal conductance of the detectors, which is mainly determined by the substrate properties, dc Joule or self heating is a major limiting factor to the use of high bias currents to obtain higher responsivity values, and hence higher values of $\mathrm{D}^{*}$. Self-heating can cause an excessive temperature rise, $\Delta \mathrm{T}_{\mathrm{dc}}$, in the film with respect to the temperature of the sample holder where the temperature sensor is normally placed. The resulting $\Delta \mathrm{T}_{\mathrm{dc}}$ may be mainly across the substrate material, or across the substrate-cold finger thermal boundary resistance. This is determined by the relative dimensions of the superconducting film pattern compared to the thickness of the substrate. The relative dimensions of the film and the substrate determine the dominating factor limiting the dc thermal conductance, $G(0)$, as discussed in details in sections 4 and 5.

For patterns with dimensions much smaller than the substrate thickness, $d_{S}, G(0)$ is limited by the lateral heat flow through the superconductive film and heat diffusion into the substrate material [5]-[6], [13], [14], [54]. This is highest for the MgO substrate samples since they have the highest thermal conductivity. In samples with pattern dimensions comparable to, or larger than their substrate thickness, $G(0)$ is limited by the thermal resistance at the substrate boundaries, and is not strongly dependent on the substrate material [24],[16]. In the studied samples, $\Delta \mathrm{T}_{\mathrm{dc}}$ is mainly at the substrate-cold finger interface, since the dimensions of the meander line patterns are comparable to that of the substrate [7]-[24].

Regardless of the dominating factor determining the total thermal conductance, $G_{t}$, the dc bias current at any temperature higher than $T_{c \text {-zero }}$ is limited by the thermal runaway characterized by the factor $\alpha$ defined in equation (4.2). As given in equation (8.3), for substrates yielding films with sharper transitions, the ultimate allowed Ib would be lower. The effect of an instability in the temperature of the film due to high bias currents, will appear as an unrealistically sharp transition in the $R$ versus $T$ curve [23], [7], [42]. The increase of the response due to the sharper transition, $\mathrm{dR} / \mathrm{dT}$, dominates the effect of this decrease in the maximum allowed $\mathrm{I}_{b}$ in (4.2), giving an overall increase in the responsivity for films with sharper transitions. The maximum bias currents, $I_{b-\max }$, of the characterized samples obtained 
Table 8.1. Thermal and electrical parameters, and dimensions of the superconducting film and substrates of the samples. D*s are obtained for $100 \mathrm{~Hz}$ modulation frequency and $20 \mu \mathrm{m}$ radiation wavelength. $r_{\max }$ is the maximum zero modulation frequency response, and $\mathrm{I}_{\mathrm{b}-\max }$ is the maximum possible bias current limited by the thermal runaway obtained for an stability factor of $\alpha=0.4$.

\begin{tabular}{lccccccccccc}
$\begin{array}{l}\text { sample } \\
\text { number }\end{array}$ & $\begin{array}{c}\text { substrate } \\
\text { material }\end{array}$ & $\begin{array}{c}\mathrm{C}_{\mathrm{S}} \\
\left(\frac{\mathrm{J}}{\mathrm{k}-\mathrm{cm}^{3}}\right)\end{array}$ & $\begin{array}{c}\mathrm{K}_{\mathrm{S}} \\
\left(\frac{\mathrm{W}}{\mathrm{k}-\mathrm{cm}}\right)\end{array}$ & $\begin{array}{c}\mathrm{d}_{\mathrm{S}} \\
(\mathrm{cm})\end{array}$ & $\begin{array}{c}\mathrm{G}(0) \\
\left(\frac{\mathrm{mW}}{\mathrm{K}}\right)\end{array}$ & $\begin{array}{c}\mathrm{dR} / \mathrm{dT} \\
\left(\frac{\Omega}{\mathrm{K}}\right)\end{array}$ & $\begin{array}{c}\mathrm{d}_{\mathrm{f}} \\
\mathrm{nm})\end{array}$ & $\begin{array}{c}\mathrm{A} \\
\left(\mathrm{cm}^{2}\right)\end{array}$ & $\begin{array}{c}\mathrm{I}_{\mathrm{b}-\max } \\
(\mathrm{mA})\end{array}$ & $\begin{array}{c}\mathrm{r}_{\max } \\
\left(\frac{\mathrm{V}}{\mathrm{W}}\right)\end{array}$ & $\begin{array}{c}\mathrm{D}^{*} \\
\left(\frac{\mathrm{cm}-\sqrt{\mathrm{Hz}}}{\mathrm{W}}\right)\end{array}$ \\
\hline $064-01$ & $\mathrm{SrTiO}_{3}$ & 0.43 & 0.052 & 0.05 & 3 & 1800 & 220 & 0.017 & 0.66 & $400 \eta$ & $3.6^{*} 10^{7}$ \\
$064-02 \mathrm{~b}$ & $\mathrm{MgO}$ & 0.53 & 3 & 0.025 & 15.5 & 950 & 120 & 0.075 & 2.08 & $127 \eta$ & $0.3^{*} 10^{6}$ \\
$064-03 \mathrm{a}$ & $\mathrm{MgO}$ & 0.53 & 3 & 0.05 & 11 & 1000 & 170 & 0.075 & 1.66 & $151 \eta$ & - \\
$064-04$ & $\mathrm{LaAlO}_{3}$ & 0.59 & 0.16 & 0.05 & 7.8 & 2200 & 190 & 0.075 & 1.0 & $274 \eta$ & $1.8^{*} 10^{6}$ \\
\hline
\end{tabular}

for $\alpha=0.4$ are given in Table 8.1. Considering the maximum $\mathrm{Ib}_{\mathrm{b}}$ determined by equation (8.3), the magnitude of maximum responsivity follows;

$\left|r_{v}\right|_{\max }=\frac{h}{G_{t}} \sqrt{\frac{\alpha G(0)}{1+(2 \pi f t)^{2}} \frac{d R}{d T}}$,

Where $\tau=C_{t} / G_{t}$ [7]. Hence from equation (8.4), devices on substrates with lower thermal conductivities and lower heat capacities, and allowing higher $\mathrm{dR} / \mathrm{dT}$ in the transition region will yield the highest responsivities. This favors $\mathrm{SrTiO}_{3}$ substrate devices compared to $\mathrm{MgO}$ and $\mathrm{LaAlO}_{3}$ ones, for the same superconducting film and substrate dimensions. This is confirmed by the measurements in the modulation frequency range of the responsivity governed by equation (8.4). The $r_{\max }$ obtained for the studied samples, and their superconducting pattern dimensions, are given in Table 8.1.

As given in equation (8.4), $\left|r_{f}\right|_{\max }$ can be increased by increasing $G(0)$, scaling as $G(0){ }^{0.5}$. This is when the value of $G_{t}$ at the operating frequency is different from that of $G(0)$ which occurs at midrange or higher frequencies [7]. At very low frequencies, lower than a critical value, $f_{l}, G_{t}$ might becomes equal to or comparable to $G(0)$. The value of $f_{1}$ depends on the substrate properties, and is the frequency at which the thermal diffusion length into the substrate approaches the substrate thickness. Then $\left|r_{f}\right|_{\max }$ is decreased by increasing $G(0)$, scaling as $G(0)^{-0.5}$. Variation of the determining factor for $G_{t}$ affects the slope of the responsemagnitude versus modulation frequency. For $f<f_{1}$ the response scales as $\mathrm{f}^{-1}$, and for $\mathrm{f}>\mathrm{f}_{1}$ scales as $\mathrm{f}^{-.5}$ [2], [7]. This is a function of substrate properties and is discussed in the following.

d) Substrate material dependent substrate-thickness effects on the responsivity limits

Considering the thermal resistance at the film-substrate interface to be negligible compared to that of the substrate material and the substrate cold-finger interface in the low and midrange frequency [7]-[24], the overall thermal conductance, $G_{t}$, is then determined by the thermal diffusion length of the absorbed energy (by the superconducting film) into the substrate, $\mathrm{L}_{\mathrm{f}}$, as defined by equation (2.8). Considering $\mathrm{L}_{\mathrm{f}}$ as the effective length for the ac heat flow into the substrate, in the modulation frequency range where $\mathrm{L}_{\mathrm{f}}$ is less than the substrate thickness (i.e. $f>f_{1}$ ), $G_{t}$ will be determined by the substrate material, and increases with the frequency, scaling as $f^{0.5}$ [7]. Hence, for a detector being operated above $f_{1}$, the substrate thickness doesn't affect the responsivity, and $\left|r_{f}\right|_{\max }$ is governed by equation (8.4), with the substrate thermal parameters the determining factors. This is valid for frequencies at 


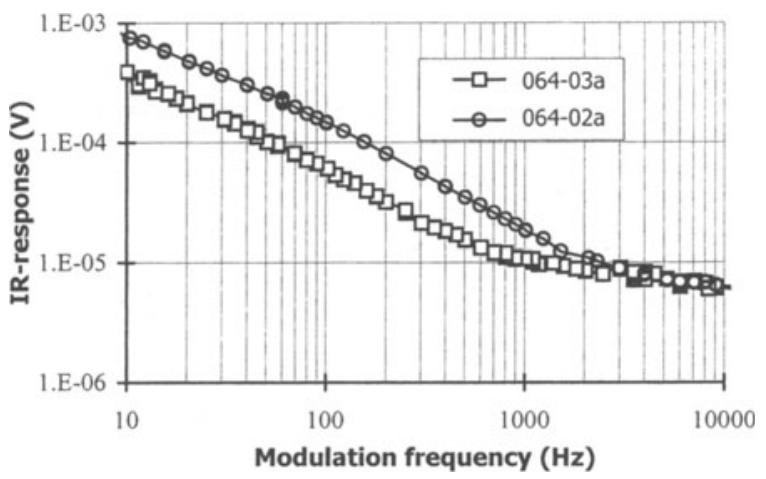

Figure 8.2. IR-response vs. modulation frequency for $0.05-\mathrm{cm}$ thick $\mathrm{MgO}$ substrate sample 064-03a and 0.025-cm thick $\mathrm{MgO}$ substrate sample 064-02a at $1 \mathrm{~mA}$ bias current.

which the thermal conductance of the superconductive film and the film-substrate interface are negligible [7].

When the frequency decreases below the values where $\mathrm{L}_{\mathrm{f}}$ becomes comparable to or larger than the substrate thickness (i.e. $f<f_{l}$ ), $G_{t}$ approaches $G(0)$ and the substrate-cold finger boundary resistance, $R_{s-c}=1 / G_{s-c}$, will be the determining factor in the total thermal conductance. In the studied samples $\mathrm{R}_{\mathrm{S}-\mathrm{c}}$ values are found to be much larger than the thermal resistance of the substrates, giving $G(0) \cong G_{s-c}$. For $(2 \pi f \tau)^{2} \gg>1$, and still valid in the above frequency range, and $G_{t} \cong G(0)$ in equation (8.4), the maximum responsivity follows;

$\left|\mathrm{r}_{\mathrm{v}}\right|_{\max }=\frac{\mathrm{h}}{2 \pi \mathrm{fC}} \mathrm{t} \sqrt{\alpha \mathrm{G}(0) \frac{\mathrm{dR}}{\mathrm{dT}}}$.

Hence, under the above conditions and within this frequency regime $\left(f<f_{1}\right)$, thinning the substrate will increase $r_{v}$ by decreasing $C_{t}$, as long as the bias current does not exceed the critical current value of the superconducting film, $I_{c}$, assuming $G(0)$ constant. The thermal boundary resistance at the substrate-cold-finger interface, $1 / \mathrm{G}(0)$, also decreases by thinning the substrate regardless of the substrate materials. [24]. For large area patterns the decrease in $G(0)$ is approximately proportional to the substrate thickness, $d_{s}$. Hence, by considering $C_{t}$ to be also proportional to the substrate material's thickness, thinning the substrate is expected to increase $\left|r_{v}\right|_{\max }$ by about $\left(d_{\mathrm{si}} / \mathrm{d}_{\mathrm{sf}}\right)^{3 / 2}$ where $\mathrm{d}_{\mathrm{si}}$ and $\mathrm{d}_{\mathrm{sf}}$ are the initial and final substrate thick nesses, respectively.

The effect of the modulation frequency on the response in different frequency regimes for two samples with identical meander line patterns on $0.025 \mathrm{~cm}$ and $0.05 \mathrm{~cm}$ thick crystalline $\mathrm{MgO}$ substrates are shown in Figure8.2. At high frequencies where $\mathrm{L}_{\mathrm{f}}$ is less than the substrate thickness for both samples, the responsivities are equal and the response is determined by the characteristics of the superconducting pattern and the substrate material. For lower frequencies where $\mathrm{L}_{\mathrm{f}}$ is larger than the thickness of the substrates, the response of the sample with thinner substrate (064-02a) is higher. This is mainly due to differences in $C_{t}$ value, being approximately proportional to the thickness of the substrates since the bias current is same. The maximum responsivity of the $0.025 \mathrm{~cm}$ thick substrate sample (064-02a) is about 2.8 times than the $0.05 \mathrm{~cm}$ thick substrate sample (064-03a) due to also the differences in $\mathrm{G}(0)$ values as well as the $C_{t}$ values. 


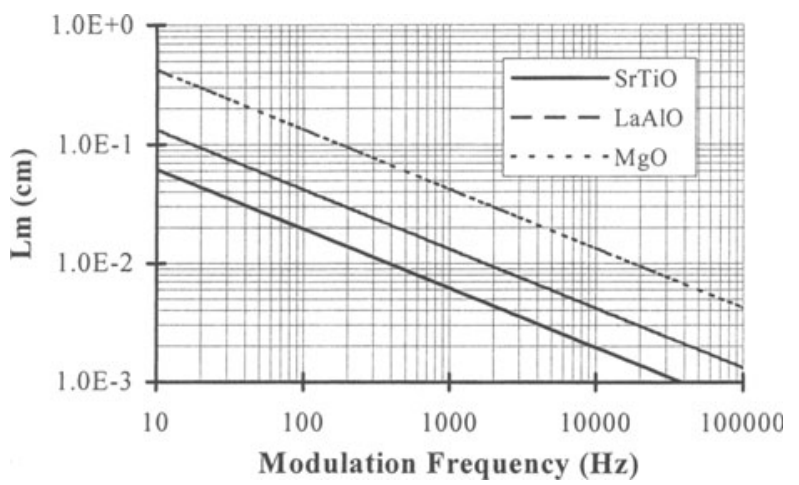

Figure 8.3. Calculated $\mathrm{L}_{\mathrm{m}}$, versus modulation frequency for bolometers on crystalline $\mathrm{SrTiO}_{3}, \mathrm{LaAlO}_{3}$, and $\mathrm{MgO}$ substrate materials.

If a detector is used in a bridge (micro-bolometer) configuration [99], $\mathrm{G}_{\mathrm{t}}$ will be primarily determined by the lateral heat flow through the substrate. Since $d_{S}$ is still much larger than the film thickness, the resulting heat flow through the film is negligible. In this configuration, thinning the substrate will also increase $r_{v}$, mainly by lowering $C_{t}$, [9], [13], [99]. Thinning the substrate in this case will cause a decrease in the $\mathrm{G}(0)$ of the detector by reducing the area of the lateral thermal conducting path through the substrate material. This gives an increase in $\mid r_{v}$ $I_{\max }$ lower than the expected value, and scaling as $\left(\mathrm{d}_{\mathrm{si}} / \mathrm{d}_{\mathrm{sf}}\right)^{0.5}$. The minimum required thickness of the substrate, $\mathrm{L}_{\mathrm{m}}$, versus modulation frequency beyond which the responsivity increases by further thinning is given for the three substrate materials in Figure 8.3.

\section{B. Spectral responsivity and substrate material dependence}

The spectral responsivity of sample 064-01a measured up to $20 \mu \mathrm{m}$, at two bias currents, is shown in Figure 8.4. The sample is made of a $220-230 \mathrm{~nm}$ YBCO superconducting film on a $0.05 \mathrm{~cm}$ thick, crystalline, $\mathrm{SrTiO}_{3}$ substrate. The superconductive pattern is a $50 \mu \mathrm{m}$ wide

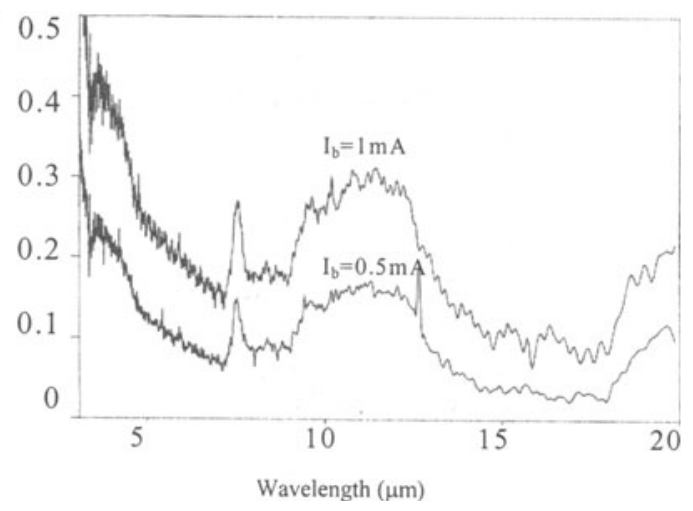

Figure 8.4. Spectral response of $\mathrm{SrTiO}_{3}$ sample 064-01a at $0.5 \mathrm{~mA}$ and $1 \mathrm{~mA}$ bias current. 
and $1.9 \mathrm{~cm}$ long meander line with a total area of $0.0168 \mathrm{~cm}^{2}$ [11]. The magnitude of the response in Figure 8.4 is proportional to $\mathrm{Ib}$, while the spectral response follows the spectral absorption of the substrate material as shown in Figure 8.5. This is due to the absorption of radiation in the open areas of the substrate between the meander lines, with the flow of heat to the superconductor. The measured spectral absorption of the $\mathrm{SrTiO}_{3}$ substrate is slightly different from that obtained using the classical dispersion theory [41], [100]-[101] particularly showing a strong peak at about $7.5 \mu \mathrm{m}$ radiation wavelength, as observed in both Figure 8.4 and Figure 8.5.

As shown in Figure 8.4, the response of the $\mathrm{SrTiO}_{3}$ substrate sample also follows the trend for the absorption in thin film and single crystal $\mathrm{YBa}_{2} \mathrm{Cu}_{3} \mathrm{O}_{7-\mathrm{x}}$ material, increasing at shorter wavelengths [10]-[33]. Using the ratio of the change in the response due to the substrate absorption with respect to that of the film, the fraction of the absorbed power by the substrate can be obtained at any wavelength. As derived for sample 064-01a $\left(\mathrm{SrTiO}_{3}\right.$ substrate), about $50 \%$ of the response at $10 \mu \mathrm{m}$ and over about $75 \%$ of the response at $20 \mu \mathrm{m}$ is due to the absorption of the incident radiation onto the open areas of the substrate. The effect of the substrate absorption on the response dominates at some longer wavelengths (i.e. $20 \mu \mathrm{m}$ ) where the absorption by the superconducting film is even further reduced [10]-[33]. The absolute value of the responsivity for the above sample is reported at $7.39 \mu \mathrm{m}$ with a 500 $\mathrm{K}$ blackbody source. A response of $1.582 \mu \mathrm{V}$ is measured for a radiation intensity of $5.64 \times 10^{-}$ ${ }^{4} \mathrm{~W} / \mathrm{cm}^{2}$ at $400 \mathrm{~Hz}$ modulation frequency, and $300 \mu \mathrm{A}$ dc bias current. A responsivity of 0.5 $\mathrm{V} / \mathrm{W}$ and a $\mathrm{D}^{*}$ of $0.72 \times 10^{6} \mathrm{~cm}-\mathrm{Hz} \mathrm{z}^{0.5} / \mathrm{W}$ are obtained under above conditions.

\section{Engineering changes for higher $D^{*}$}

As an example of engineering changes required to increase $D^{*}$ using the analysis presented in this work, an analysis for the sample on $\mathrm{SrTiO}_{3}$ substrate is discussed. From the spectral response in Figure 8.4 and by use of a maximum bias current of $0.66 \mathrm{~mA}$ from Table 8.1 , a D* of about $3.6 \times 10^{7} \mathrm{~cm}-\mathrm{Hz}^{0.5} / \mathrm{W}$ is obtained for the $\mathrm{SrTiO}_{3}$ substrate sample (064-01a), at $20 \mu \mathrm{m}$ wavelength, and $100 \mathrm{~Hz}$ modulation frequency. The spectral detectivity, at a constant bias current and temperature, follows the spectral response of the detector for a constant radiation intensity. The $\mathrm{D}^{*}$ of some samples at $20 \mu \mathrm{m}$ radiation wavelength is given

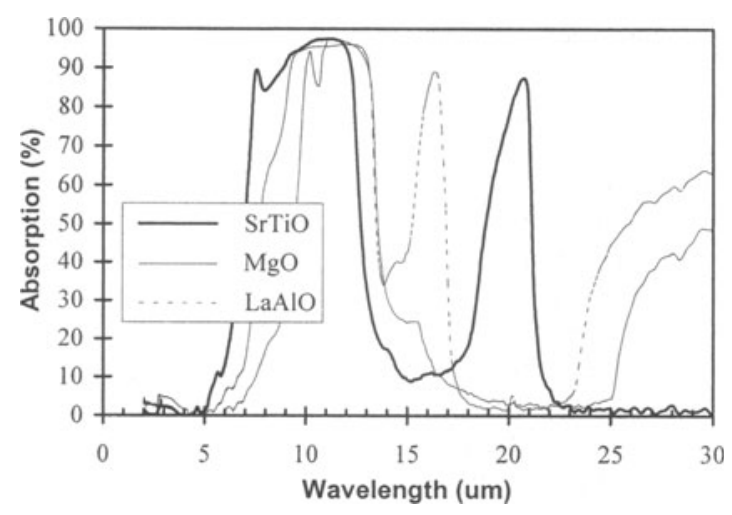

Figure 8.5. Measured spectral absorption of 0.05 -cm thick crystalline $\mathrm{SrTiO}_{3}$, $\mathrm{MgO}$, and $\mathrm{LaAlO}_{3}$ substrates. 
in Table 8.1.

To increase $\mathrm{D}^{*}$, considering the $400 \mathrm{~Hz}$ and $100 \mathrm{~Hz}$ modulation frequency points in Figure 8.3 as an example, the substrate of sample 064-01 a should be thinned to below $0.01 \mathrm{~cm}$ and $0.02 \mathrm{~cm}$ respectively. The substrate can be thinned using wet etching after the patterning process for the superconductive film. Thinning the substrate will also increase $G(0)$, while it is still limited by $R_{s-c}$. Based on above analysis, by thinning the substrate down to $0.005 \mathrm{~cm}$, with an increase of the total area of superconductive pattern up to $0.09 \mathrm{~cm}^{2}$, a D* on the order of $1 \times 10^{9} \mathrm{~cm}-\mathrm{Hz}^{0.5} / \mathrm{W}$ at $20 \mu \mathrm{m}$ for $200 \mathrm{~nm}$ thick $\mathrm{YBa}_{2} \mathrm{Cu}_{3} \mathrm{O}_{7-\mathrm{x}}$ superconducting films on crystalline $\mathrm{SrTiO}_{3}$ substrates will be obtained. Also as observed from equation (8.2) and Table 8.1 , the detectivity of samples can be enhanced by a sharper transition (favored by the substrate lattice structure), since it allows lower $\mathrm{I}_{\mathrm{b} \text {-max }}$ values, and hence lower current dependent noise in the region.

\section{SUMMARY AND CONCLUSIONS}

To explain the behaviors of edge transition bolometers, it is necessary to include in the modeling, the effects of thermal boundary resistances, temperature dependence of the thermal parameters of the devices, the frequency dependence of the thermal diffusion length into the materials, noise, and artifacts due to the bias configurations and ambient atmosphere. Such models are studied that explain the modulation frequency dependence in the low and midrange frequencies within the range of interest for contemplated applications of bolometers.

For the samples with large area superconducting film patterns compared to the substrate thickness, the thermal boundary resistance at the substrate-holder of the samples is considerably higher than that of the other sources as film-substrate boundary resistance, the substrate material resistance, and the bulk film thermal resistance. This is the case only at very low modulation frequencies. In such a configuration the response of the samples follow a basic two-lumped element R-C model. Then, $\mathrm{G}$ is mainly determined by the thermal boundary resistance at the substrate-holder interface, and $\mathrm{C}$ is mainly due to the heat capacity of the substrate materials. But this is up to frequencies where the thermal diffusion length, $\mathrm{L}$, into the substrate material is more than the substrate thickness, $\mathrm{L}_{\mathrm{s}}$. Several methods for measuring the thermal conductance $\mathrm{G}$, has been introduced.

The increase of the modulation frequency beyond the values where $\mathrm{L}<\mathrm{L}_{\mathrm{S}}$, causes a change in the slope of the response magnitude versus modulation frequency compared to that of the basic R-C model. This happens at a "knee" frequency, $\mathrm{f}_{\mathrm{L}}$, which is dependent on the substrate material and its thickness. This leads to different regime of frequency response behavior. For frequencies less than $f_{L}$, the response scales as $\mathrm{f}^{1}$ following the response for a basic $\mathrm{R}-\mathrm{C}$ model. For frequencies higher than $\mathrm{f}_{\mathrm{L}}$, the response scales as $\mathrm{f}^{0.5}$ due to the variation of the effective penetration depth of the temperature variation into the substrate. This is up to the frequencies where the thermal resistance of the superconducting film-substrate boundary, $\mathrm{R}_{\mathrm{fs}}$, is negligible compared to the frequency dependent effective thermal conductance of the substrate materials, $R_{s}$. In the regime of the $\mathrm{f}^{0.5}$ dependence for the responsivity, thinning the substrate underneath the pattern to increase the responsivity is dependent on the thermal diffusion length.

Thinning the substrate of the samples to increase the maximum responsivity, $\left|r_{v}\right|_{\max }$, depends on the variations of the total thermal conductance, $G_{t}$, and the total heat capacitance of the device, $\mathrm{C}_{\mathrm{t}}$. The critical required thinning of the substrates below which the responsivity increases is dependent on the substrate materials and the modulation frequency, $f$, scaling as $f$ 
0.5. In low enough frequency regime, where the thermal diffusion length into the substrate is higher than the substrate thickness, $L_{\mathrm{f}}>\mathrm{d}_{\mathrm{s}}$, thinning substrate of the detector increases $\left|r_{v}\right|_{\max }$ by a factor of $\left(\mathrm{d}_{\mathrm{si}} / \mathrm{d}_{\mathrm{sf}}\right)^{3 / 2}$, where $\mathrm{d}_{\mathrm{si}}$ and $\mathrm{d}_{\mathrm{sf}}$ are the initial and final substrate thicknesses, respectively. In the higher frequency regime where $\mathrm{L}_{\mathrm{f}}<\mathrm{d}_{s}$, thinning the substrate of the detector increases $\left|\mathrm{r}_{\mathrm{v}}\right|_{\max }$ by about $\left(\mathrm{d}_{\mathrm{si}} / \mathrm{d}_{\mathrm{sf}}\right)^{0.5}$.

The film-substrate thermal boundary resistance, $R_{b d}$, is not effective on the IR-response in the thin film samples with in the studied modulation frequencies here. Considering a film thickness of $200 \mathrm{~nm}$, the $\mathrm{R}_{\mathrm{bd}}$ would be effective above about $100 \mathrm{kHz}, 1 \mathrm{MHz}$, and $10 \mathrm{MHz}$, in $\mathrm{MgO}, \mathrm{LaAlO}_{3}$, and $\mathrm{SrTiO}_{3}$, respectively which is mainly determined by the thermal parameters of the substrate material. The $\mathrm{R}_{b d}$ is effective at much lower frequency range of $\mathrm{KHz}$ for the screen-printed thick film samples, which have values of thickness typically in the range of $10 \mathrm{~s}$ of micrometers. To improve the responsivity of the bolometer in the various regimes of frequency, for a fixed transition region width, thinner films with narrow line width and a long meander line are favored (to increase $\mathrm{dR} / \mathrm{dT}$ ). For high absorption in the film, a minimum thickness of about $150 \mathrm{~nm}$ is also needed [I_7]. The response of the thick film Y$\mathrm{Ba}-\mathrm{Cu}-\mathrm{O}$ bolometers mostly exhibits a bolometric response at their transition temperature. This is while small response, relative to the bolometric response at the transition of the films, is reported at temperatures right below the $\mathrm{T}_{\mathrm{c}(\mathrm{zero})}$ under certain conditions. This response is attributed to the noise signal due to the Kosterlitz-Thouless effect, which is strongly dependent on the bias current. The thick film samples are typically made of post-annealed screen-printed films and hence are highly granular.

A one-dimensional model based on the above facts, which is for bolometers with superconducting film-patterns larger or comparable to the substrate thickness, is described. The model while being a useful tool for design optimizations for such devices could also be used to monitor the thermal parameters of the detectors and the used materials. A very good fit between the both measured magnitude and phase of the response to that obtained from the model is obtained for the samples from very low modulation frequencies of $0.5 \mathrm{~Hz}$ up to about $100 \mathrm{kHz}$, the studied range in this chapter. The phase of response versus modulation frequency of the bolometers is a much more sensitive characteristic to the thermal parameters compared to the magnitude of the response. Using the model and from the low and midrange frequency of the response, the heat capacity and thermal conductivity of the used substrate materials and the substrate-holder thermal boundary resistance can also be obtained.

Simultaneous measurements of the IR-response and $\mathrm{dR} / \mathrm{dT}$ versus temperature reveal a discrepancy between the magnitude of the response and the measured $\mathrm{dR} / \mathrm{dT}$ of the bolometers, which is frequency dependent. This is verified to be apart from the artifacts caused by the Joule heating due to the bias current and the microstructure of the films. Experimental data on the phase of the response variation suggest a change of the thermal conductivity of the substrate material as a source for the reported discrepancy. This is through the radiation limit in phonon propagation. Due to enhancement of the mean free path of phonons emitted from the superconducting film, the limit thermal conductance at $\mathrm{T}<\mathrm{T}_{\mathrm{c} \text {-onset }}$ is increased. The influence of the superconducting state on the thermal parameters of the bolometers is also conducted. Different modulation frequency regimes is analyzed and good agreement between the theoretical calculations with experimental data is obtained in particular for low modulation frequencies at temperatures closer to $T_{\text {c-zero. }}$.

The noise characteristics of one sample can't be fully predicted, and may differ strongly from one sample to another. Four different noise characteristics are identified at the transition temperature of the superconductor. Since a superconducting bolometer is operated at the 
maximum $\mathrm{dR} / \mathrm{dT}$, the noise characteristics in the transition region are of main interest in the design of devices. Two types of the four identified noise signals are identified to be in the transition region of interest. One is excess noise over a region at $T_{\text {c-zero }}$, which has the characteristics of a white noise. It peaks at $\mathrm{T}_{\mathrm{c} \text {-zero }}$ and widens with an increase in the bias current, extending to lower temperatures. This noise is attributed to the weak links and the corresponding Josephson junctions (SNS) at the grain boundaries. The onset temperature of this type of noise versus bias current has a behavior similar to the critical current density, $\mathrm{J}_{\mathrm{c}}$, versus temperature. This is observed in samples, which are highly granular and have a wide transition.

The second type of noise peaks at the middle of the transition and is strongly dependent on the bias current and frequency. Hence, this is attributed to conductivity fluctuations in the region of the superconducting transition. This type of fluctuations is interpreted to be dominated by the conductivity fluctuations, due to the variations in the volume fraction of the superconducting phase, in the current path through the material.

The substrate absorption is also a determining factor in the spectral responsivity, and hence in the detectivity of the detectors. This is in particular when the open areas of the substrate are comparable or larger than the absorbing surface area of the superconducting film. For an YBCO edge-transition bolometer operating at wavelengths from the near infrared to the far infrared, the spectral absorption of the substrate material is a major factor since the absorption of the superconducting films is strongly reduced compared to its values at shorter radiation wavelengths. This property of the substrates can be used for selective wavelength sensitivity for an edge-transition superconductor infrared detector.

In conclusion, the studied models and the analysis in this chapter can be used to monitor the thermal parameters of the superconducting film, substrate, and the thermal boundary resistance values of an edge-transition superconductive bolometer. Also the mechanism of heat flow caused by the absorbed radiation in the samples can be investigated by use of the results from the analysis in this kind of devices. The response versus modulation frequency of the bolometers is the most informative data in this respect. The response of the bolometers with high quality large superconducting pattern areas compared to the substrate thickness is concluded to be mainly bolometric at low and midrange modulation frequencies. Consideration of a three dimensional solution to the model would be required for further accurate response analysis of samples with small superconducting pattern areas compared to the substrate thickness.

\section{REFERENCES}

[1] P. L. Richards, J. Clark, R. Leoni, Ph. Lerch, S. Verghese, M. R. Beasley, T. H. Geballe, R. H. Hammond, P. Rosenthal, and S. R. Spielman, "Feasibility of the high $\mathrm{T}_{\mathrm{C}}$ superconducting bolometer", Appl. Phys. Lett., vol. 54, no. 3, pp. 283-285, 1989.

[2] Qing $\mathrm{Hu}$ and P. L. Richards, "Design analysis of a high $\mathrm{T}_{\mathrm{c}}$ superconducting microbolometer", Appl. Phys. Lett., vol. 55, no. 23, pp. 2444-2446, 1989.

[3] J. Schutzmann, W. Ose, J. Keller, K. F. Renk, B. Roas, L. Schultz, and G. SaemannIschenko, "Far-infrared reflectivity and dynamical conductivity of an epitaxial $\mathrm{YBa}_{2} \mathrm{Cu}_{3} \mathrm{O}_{7-\delta}$ thin film", Europhys. Lett., vol. 8, pp. 679-684, 1989. 
[4] M. Fardmanesh, M. Ihsan, A. Rothwarf, K. Scoles, and K. Pourrezaei, " Thick and thin film Y-Ba-Cu-O infrared detectors", American Institute of Physics Proceeding, no. 251, pp. 681-691, 1991.

[5] M. Nahum, Qing Hu, P. L. Richards, S. A. Sachtjen, N. Newman and B. F. Cole, " Fabrication and Measurement of High $\mathrm{T}_{\mathrm{c}}$ Superconducting Bolometers", IEEE Trans. on Mag., vol. 27, pp. 3081-3084, 1991.

[6] U. P. Oppenheim, M. Katz, G. Koren, E. Polturak and M. R. Fishman, "High temperature superconducting bolometer", Physica C, vol. 178, pp. 26-28, 1991.

[7] M. Fardmanesh, A. Rothwarf, and K. J. Scoles, "Low and Midrange Modulation Frequency Response for YBCO Infrared Detectors: Interface Effects on the Amplitude and Phase", IEEE Trans. on Appl. Supercond., Vol. 5, no. 1, pp. 7-13, March 1995.

[8] S. Zeuner, W. Prettl, and H. Lengfellner, " Fast thermoelectric response of normal state $\mathrm{YBa}_{2} \mathrm{Cu}_{3} \mathrm{O}_{7-d}$ films", Appl. Phys. Lett., Vol. 66, no. 14, pp. 1833-1835, 1995.

[9] M. Fardmanesh and A. Rothwarf, "Control of superconductive edge transition $\mathrm{YBa}_{2} \mathrm{Cu}_{3} \mathrm{O}_{7-\mathrm{x}}$ bolometers responsivity and detectivity through substrate properties," Applied Optics, Vol. 38, No. 19, 1999.

[10] Z. M. Zhang, B. I. Choi, T. A. Le, M. I. Flik, M. P. Siegal and J. M. Phillips, "Infrared refractive index of thin $\mathrm{YBa}_{2} \mathrm{Cu}_{3} \mathrm{O}_{7}$ superconducting films", J. Heat Transfer, vol. 114, pp. 644-652, 1992.

[11] M. Fardmanesh, A. Rothwarf, and K. J. Scoles, "Noise characteristics and detectivity of $\mathrm{YBa}_{2} \mathrm{Cu}_{3} \mathrm{O}_{7}$ superconducting bolometers: Bias current, frequency, and temperature dependence," J. Appl. Phys., Vol. 79, no. 4, pp. 2006-2011, 1996.

[12] K. D. Irwin, G. C. Hilton, D. A. Wollman, and John M. Martinis, "X-ray detection using a superconducting transition-edge sensor microcalorimeter with electrothermal feedback, " Appl. Phys. Lett. Vol 69, no. 13, pp. 1945-1947, 1996.

[13] G. Darcy Poulin, Jean Lachapelle, Steven H. Moffat, Frank A. Hegmann, and John S. Preston, "Current-Voltage characteritics of dc voltage biased high temperature superconducting microbridges," Appl. Phys. Lett., Vol. 66, no. 19, pp. 2576-2578, 1995.

[14] A. V. Sergeev, A. D. Semenov, P. Kouminov, V. Trifonov, I. G. Goghize, B. S. Karasik, G. N. Gol'tsman, and E. M. Gershenzon, "Transparency of a $\mathrm{YBa}_{2} \mathrm{Cu}_{3} \mathrm{O}_{7}$ film/substrate interface for thermal phonons measured by means of voltage response to radiation," Phys. Rev. B, Vol. 49, no. 13, pp. 9091-9096, 1994.

[15] C. D. Marshal, I. M. Fishman, R. C. Dorfman, C. B. Eom, and M. D. Fayer, "Thermal diffusion, interfacial thermal barrier, and ultrasonic propagation in $\mathrm{YBa}_{2} \mathrm{Cu}_{3} \mathrm{O}_{7-\mathrm{x}}$ thin films: Surface-selective transient-grating experiments", Physical Review B, Vol. 45, no. 17, pp. 10 009-10 021, 1992.

[16] P. E. Phelan, "Thermal response of thin-film high- $T_{c}$ superconductors to modulated irradiation," J. of Therm. Phys. Heat Transfer, Vol. 9, no. 3, pp 397-402, 1995.

[17] M. Nahum, S. Verghese, and P. L. Richards, "Thermal boundary resistance for $\mathrm{YBa}_{2} \mathrm{Cu}_{3} \mathrm{O}_{7-\mathrm{x}}$ films", Appl. Phys. Lett., Vol. 59, no. 16, pp. 2034-2036, 1991.

[18] M. Fardmanesh, "Analytic Thermal Modeling for dc to Midrange Modulation Frequency Response of Thin Film High-Tc Superconductive Edge-Transition Bolometers," Applied Optics; Optical Technology \& Biomedical Optics, Vol. 40, No. 7, pp. 1080-1088, 2001. 
[19] S. Bhattacharya, X. X. Xi, M. Rajeswari, C. Kwon, S. N. Mao, Qi Li, and T. Venkatesan, "Optical response of an ultrathin film and a large-angle grain-boundary in superconducting $\mathrm{YBa}_{2} \mathrm{Cu}_{3} \mathrm{O}_{7-\mathrm{x}}$," J. Appl. Phys., Vol. 62, no. 26, pp. 3510-3512, 1995.

[20] Kin Li, and Joseph E. Johnson, "Influence of substrate and biasing current on response of YBCO microbolometers," IEEE Trans. on Appl. Supercond., Vol. 3, no. 1, pp. 2111-2114, 1993.

[21] F. A. Hegmann, and J. S. Preston, "Identification of nonbolometric photoresponse in $\mathrm{YBa}_{2} \mathrm{Cu}_{3} \mathrm{O}_{7-\mathrm{x}}$ thin films based on magnetic field dependencd," Appl. Phys. Lett., Vol. 62, no. 10, pp. 1158-1160, 1993.

[22] J. H. Hao, F. Q. Zhou, H. D. Sun, X. J. Yi, and Z. G. Li, "Responsivity calculation and measurement of $\mathrm{YBaCuO}$ optical detector," IEEE Trans. on Appl. Supercond., Vol. 3, no. 1, pp. 2167-2169, 1993.

[23] Hsiung Chou, ,H. Z. Chen, M. T. Hong, Y. C. Chen, and T. C. Chow, "Bolometric detection in a precipitation free $\mathrm{YBa}_{2} \mathrm{Cu}_{3} \mathrm{O}_{7-\mathrm{x}}$ film at $77 \mathrm{~K}, "$ Appl. Phys. Lett., Vol. 68 , no. 19 , pp. 2741-2743, 1996.

[24] M. Fardmanesh, A. Rothwarf, and K. J. Scoles, "YBa2 $\mathrm{Cu}_{3} \mathrm{O}_{7-\mathrm{x}}$ infrared bolometers: temperature dependent responsivity and deviations from the $\mathrm{dR} / \mathrm{dT}$ curve.", J. Appl. Phys., Vol. 77, no. 9, pp. 4568-4575, 1995.

[25] M. Fardmanesh, K. J. Scoles, and A. Rothwarf, "DC charateristics of patterned $\mathrm{YBa}_{2} \mathrm{Cu}_{3} \mathrm{O}_{7-\mathrm{x}}$ superconducting thin film bolometers: Artifacts related to Joule heating, ambient pressure, and microstructure," IEEE Trans. on Appl. Supercond., Vol. 8, no. 2, pp. 69-78, 1998.

[26] M. Fardmanesh, M. Ihsan, K. Scoles, and A. Rothwarf, "Thin film YBCO infrared detector design and characterization", American Institute of Physics Proceeding, no. 273, pp. 142-154, 1992.

[27] M. Thsan, M. Fardmanesh, A. Rothwarf, K. Pourrezaei, and E. Lenzing, "Design and performance of microstrip resonators using YBCO thin films made by in-situ off-axis sputtering", American Institute of Physics Proceeding, no. 273, pp. 122-131, 1992.

[28] J. Schubert, M. Siegert, M. Fardmanesh, W. Zander, M. Prompers, Ch. Buvhal, Judit Lisoni, C. H. Lei, "Superconducting and Electro-optical Thin Films Prepared By Pulsed Laser Deposition Technique," Appl. Surface Science, Vol. 168, pp. 208-214, 2000.

[29] M. Fardmanesh, K. Scoles, A. Rothwarf, E. Sanchez, E. Brosha, P. Davies, "ANNEALING STUDY OF YBa2Cu3Ox AND YBa2Cu4Ox SCREEN PRINTED THICK FILMS", Amer. Inst. Phys. Proc., No. 251, pp. 283-291, 1991.

[30] Flik, M. I., Phelan, P.E., and Tien C. L., "Thermal model for the bolometric response of high $\mathrm{T}_{\mathrm{c}}$ superconducting films to optical pulses", Cryogenics, vol. 30, pp. 1118$1128,1990$.

[31] Neff, H., "Modeling and optimization of high-T $\mathrm{c}_{\mathrm{c}}$ superconducting bolometers", $J$. Appl. Phys., vol. 69, no. 12, pp. 8375-8379, 1991.

[32] D. Robbes, P. Langlois, C. Dolabjian, D. Bloyet, J. F. Hamet, and H. Murray, "YBCO microbolometer operating below $\mathrm{T}_{\mathrm{c}}$ : A modelization based on critical-temperature dependence", IEEE Trans. on Applied Superconductivity, vol. 3, no. 1, pp. 2120-2123, 1993. 
[33] D. Van der Marel, H.-U. Habermeier, D. Heitmann, W. Konig, and A. Wittlin, " Infrared study of the superconducting phase transition in $\mathrm{YBa}_{2} \mathrm{Cu}_{3} \mathrm{O}_{7-\mathrm{x}}$ ", Physica $C$, vol. 176, pp. 1-18, 1991.

[34] S. L. Bud'ko, H. H. Feng, M. F. Davis, J. C. Wolfe, and P. H. Hor, "Persistent photoconductivity in insulating and superconducting $\mathrm{YBaCuO}$ thin films: temperature and spectral dependence", Physical Review B, vol. 48, pp. 16707-16713, 1993.

[35] K. Fushinobu, P. E. Phelan, K. Hijikata, T. Nagasaki, and M. I. Flik, "Thermal analysis of the performance of a high- $\mathrm{T}_{\mathrm{c}}$ superconducting bolometer", J. Heat Transfer, Vol. 116, pp. 275-279, 1994.

[36] A. Frenkel, "Mechanism of nonequilibrium optical response of high-temperature superconductors", Physical Review B, Vol. 48, no.13, pp. 9717-9725, 1993.

[37] M. Fardmanesh, A. Rothwarf, and Kevin J. Scoles, "The responsivity and detectivity limits for patterned $\mathrm{YBa}_{2} \mathrm{Cu}_{3} \mathrm{O}_{7-\mathrm{x}}$ superconductive IR-detectors," 6th Int. Superconductive Electronics Conference proc., Vol. 3, pp. 399-401, 1997.

[38] Agha Jahanzeb, Christine M. Travers, Zeynep Celik-Butler, and Donald P. Butler, "A semiconductor $\mathrm{YBaCuO}$ microbolometer for room temperature IR imaging," IEEE Trans. on Electron Devices, Vol. 44, no. 10, pp. 1795-1801, 1997.

[39] Christine M. Travers, Agha Jahanzeb, Donald P. Butler, and Zeynep Celik-Butler, "Fabrication of semiconducting $\mathrm{YBaCuO}$ surface-micromachined bolometer arrays," J. Microrelectro-mechanical Systems, Vol. 6, no. 3, pp. 271-276, 1997.

[40] Z. M. Zhang and A. Frenkel, "Thermal and nonequilibrium responses of superconductors for radiation detectors," J. Superconductivity, Vol. 7, no. 6, pp. 871$883,1994$.

[41] K. kamaras, K. L. Barth, F. Keilmann, R. Henn, M. Reedyk, C. Thomsen, M. Cardona, J. Kircher, P. L. Richards, and J. L. Stehle, "The low-temperature infrared optical functions of SrTiO determined by reflectance spectroscopy and spectroscopic ellipsometry," J. Appl. Phys., Vol. 78, no. 2, pp. 1235-1240, 1995.

[42] D. Janik, D. May, H. Wolf, and R. Schneider, "High- $T_{c}$ transition-edge bolometer for detecting guided millimeter waves," IEEE Trans. on Appl. Supercond., Vol. 3, no. 1, pp. 2148-2151, 1993.

[43] M. I. Flik, and C. L. Tien, J. Appl. Phys., Vol. 67, no. 1, pp. 362-370, 1990.

[44] P. L. Richards, S. Verghese, T. H. Geballe, and S. R. Spielman, "The High- $\mathrm{T}_{\mathrm{C}}$ superconducting bolometer," IEEE Trans. on Mag., Vol. 25, 2, pp 1335-1338, 1989.

[45] E. Zeldov, N. M. Amer, G. Koren, and A. Gupta, "Nonbolometric optical response of epitaxial films," Phys. Rev. B, Vol. 39, no. 13, pp. 9712-9714, May 1989.

[46] Q. P. Li, "Theory of effect of uniaxial-stress on Tc in ,YBa2Cu3O7" Physica C, Vol. 209, no. 4, pp. 513-515, 1993.

[47] Q. Xiong, Y. Y. Xue, Y. Y. Sun, P. H. Hor, C. W. Chu, M. F. Davis, J. C. Wolfe, S. C. Deshmukh and D. J. Economou, "Effect of pressure on the critical current-density of YBa2Cu3O7 thin-films," Physica C, Vol. 205, no. 3, pp. 307-310, 1993.

[48] B. H. Moeckly, D. K. Lathrop, and R. A. Buhrman, "Electromigration study of oxygen disorder and grain-boundary effects in $\mathrm{YBa}_{2} \mathrm{Cu}_{3} \mathrm{O}_{7}$ thin films," Physical Review B, Vol. 47, no. 1, pp. 400-417, 1993.

[49] A. V. Sergeev, and M. Y. Reitzer, "Photoresponse mechanisms of thin superconducting films and superconducting detectors," Int. J. of Modern Phys. B, Vol. 10, no.6, pp. 635-667, 1996. 
[50] A. V. Gurevich and R. G. Mints, "Self-heating in normal metals and superconductors," Rev. Mod. Phys., Vol. 59, no. 4, pp. 941-999, 1987.

[51] A. Sh. Fix, I. L. Maximov, K. V. Morozov, and V. V. Opsipov, IEEE Trans. on Appl. Supercond., Vol. 3, p. 1608, 1993.

[52] W. M. Huber, B. Arendt, P. G. Huggard, and W. Prettl, "Square-law Josephson detection of far infrared radiation with current-biased granular high-Tc thin films," Supercond. Sci. Tech., Vol. 8, no. 10, pp. 769-773, 1995.

[53] Sung Joon Bae, Seungbum Hong, Eunah Kim, Woosuck Shin, Chang Hyun Cho, Young Kun Kim, Yong Soo Bea, Tae-Hyun Sung, and Kwangsoo No, "Effect of texturing and microbridge length on the responsivity of $\mathrm{YBa} 2 \mathrm{Cu} 3 \mathrm{Ox}$ thin film," Jpn. J. Appl. Phys., Vol. 35, no. 3, pp. 1716-1719, 1996.

[54] Q. Li, D. B. Fenner, W. D. Hamblen, and D. G. Hamblen, Appl. Phys. Lett., 62, p. 2428, 1993.

[55] J. C. Braunas and B. Lakew, Appl. Phys. Lett., 64, 777 (1994).

[56] B. J. Berkwitz, A. S. Hirahara, K. Char and E. N. Grossman, Appl. Phys. Lett., 69, p. $2125,1996$.

[57] N. Bluzer, J. Appl. Phys., 78, p. 7340, 1995.

[58] H. Kraus, Supercond. Sci. and Technology, 9, p. 827, 1996.

[59] S. F. Karmanenko, A. A. Semenov, Ia Khrebtov, Vn Leonov, TH Johansen, Yu M. Galperin, A. V. Bobyl, A. I. Dedoboretz, M. E. Gaevski, A.V. Lunev, R. A. Suris, Supercond. Sci. and Technology, 13, p. 273, 2000.

[60] T.-L. Hwang, S. E. Schwarz, and D. B. Rutledge, Appl. Phys. Lett., 34, p. 773, 1979.

[61] T. H. K. Barron, W. T. Berg, and J. A. Morrison, Royal Society Proc., A 250, p70, 1959.

[62] E. T. Swartz and R. O. Pohl, Rev. Mod. Phys., 61, p. 605, 1989.

[63] N. Bluzer, Phys. Rev. B, 44, p. 10222, 1991.

[64] J.-J. Chang and Scalapino, Phys. Rev. B, Vol. 15, no. 5, 1977.

[65] M. Tinkham, Introduction to superconductivity (Kreger, Malabar, 1985).

[66] D. Pines , Physica B, 199, p. 300, 1994.

[67] R.A.Richardson, S.D.Peacor, C.Uher, F.Nori J. Appl. Phys., 72, p. 4788, 1992.

[68] M. Fardmanesh, I. N. Askerzade, "Temperature dependence of phase of the response of YBCO Edge Transition Bolometers: effects of superconductivity transition and thermal Parameters," Superconductor Sci. Technol., Vol. 16, No. 1, pp. 28-32, 2003.

[69] M. Tinkham, and C. J. Lobb, Solid State Phys. 42, p. 91, 1988.

[70] B. W. Ricketts, R. Driver and H. K. Welsh, Solid State Commun. 67, p. 133, 1988.

[71] L. B. Kiss, P. Svedlindh, L. Lundgren, J. Hudner, H. Ohlsen, L. Stolt, and Z. Gingl, Physica B 172, pp. 441-444, 1991.

[72] A. Frenkel, M. A. Saifi, T. Venkatesan, P. England, X. D. Wu, and A. Inam, J. Appl. Phys. 67, p. 3054, 1990.

[73] P. Dutta, and P. M. Horn, Rev. Mod. Phys. 53, p. 497, 1981; M. B. Weissman, Rev. Mod. Phys. 60, p. 537, 1988.

[74] G. Jung, S. Vitale, J. Konopka, and M. Bonaldi, J. Appl. Phys. 70, p. 5440, 1991.

[75] K. H. Han, M. K. Joo, H. J. Shin, Sung-Ik Lee, and Sung-Ho Suck Salk, IEEE Transaction on Applied Superconductivity, 3, 1, pp. 2947-2949, 1993.

[76] L. B. Kiss, T. Larsson, P. Svedlindh, H. Ohlsen, M. Ottosson, J. Hudner, and L. Stolt, Physica C, 207, pp. 318-332, 1993. 
[77] R. D. Black, L. G. Turner, A. Mogro-Campero, T. C. McGee, and A. L. Robinson, Appl. Phys. Lett. 55, 21, pp. 2233-2235, 1989.

[78] J. Hall, H. Hickman, and T. M. Chen, Solid State Communications, 76, 7, pp. 921922, 1990.

[79] R. W. Zindler, and R. R. Parsons, J. Vac. Sci. Technol. A 10, 5, pp. 3322-3324, 1992.

[80] L. B. Kiss, P. Svedlindh, L. Lundgren, J. Hudner, H. Ohlsen, L. Stolt, Solid State Commun. 75, p. 747, 1990.

[81] G. Forgacs, L. S. Schulman, L. B. Kiss, P. Svedlindh, and L. Lundgren, Physica C 177, p. 67, 1991.

[82] M. Celasco, M. Lo Bue, A. Masoero, P. Mazzetti, and A. Stepanescu, IEEE trans. Appl. Supercond., AS-31, p. 111, 1993.

[83] A. Maeda, Y. Nakayama, S. Takebayashi, K. Uchinokura, Phys. C, 160, p. 443, 1989.

[84] A. Maeda, H. Watanabe, I. Tsukada, and K. Uchinokura, in Noise in Physical Systems and $1 / f$ Fluctuations. Edited by T. Musha, S. Sato, and M. Yamamoto, p. 115, Ohmsha, Tokyo, 1991.

[85] A. E. Khalil, Physica C, 196, p. 357, 1992.

[86] Yi Song, A. Misra, P. P. Crooker, and J. R. Gains, Phys. Rev. B 45, p. 7574, 1992.

[87] M. J. Ferrari et al., Phys. Rev. Lett. 64, p. 72, 1990.

[88] C. T. Rogers, K. E. Myers, J. N. Eckstein, I. Bozovic, Phys. Rev. Lett. 69, p. 160, 1992.

[89] H. J. Jensen, A. Brass, and A. J. Berlinsky, Phys. Rev. Lett. 60, p. 56, 1988.

[90] H. J. Jensen, y. Brechet, and A. Brass, J. Low Temp. Phys. 74, p. 293, 1989.

[91] E. R. Nowak, N. E. Israeloff, and A. M. Goldman, Submitted to physical Review Letter, 1993.

[92] M. Tinkham, Introduction to Superconductivity. McGraw Hill, New York, 1975.

[93] Z. M. Zhang, B. I. Choi, T. A. Le, M. I. Flik, M. P. Siegal and J. M. Phillips, 1991 ASME Winter Annual Meeting, Atlanta, GA, December 1-6, 1991.

[94] K. E. Goodson and M. I. Flik, "Electron and Phonon Thermal Conduction in Epitaxial High-Tc Superconducting Films," National Heat Transfer Conference, Minneapolis, Minnesota, July 1991.

[95] M. I. Flik, Z. M. Zhang, K. E. Goodson, M. P. Siegal, and J. M. Phillips, Phys. Rev. B, 46, pp. 5606-5614, 1992.

[96] R. T. Collins, Z. Schlesinger, F. Holtzberg, and C. Feild, Phys. Rev. Lett., 63, pp. $422-$ 425, 1989.

[97] S. L. Cooper, G. A. Thomas, J. Orenstien, D. H. Rapkine, M. Capizzi, T. Timusk, A. J. Millis, L. F. Schneemeyer, and J. V. Waszczak, Phys. Rev. B, 40, pp. 11358-11361, 1989.

[98] Julia M. Phillips, "Substrate selection for high-temperature superconducting thin films,” J. Appl. Phys., Vol. 79, no. 4, pp.1829-1848, 1996.

[99] T. G. Stratton, B. E. Cole, P. W. Kruse, R. A. Wood, K. Beauchamp, T. F. Wang, B. Johnson, and A. M. Goldman, "High-temperature superconducting bolometer," Appl. Phys. lett., Vol. 57, no. 1, pp. 99-100, 1990.

[100] A. S. Barker, Jr., and M. Tinkham, "Far-infrared ferroelectric vibration mode in $\mathrm{SrTiO}_{3} "$ Physical Review., Vol. 125, no. 5, pp. 1527-1530, 1962.

[101] J. C. Galzerani R. S. Katiyar, "The infrared reflectivity in SrTiO and the antidistortive transition," Solid State Communications., Vol. 41, no. 7, pp. 515-519, 1989. 